\title{
CLASSES DE NEVANLINNA SUR UNE INTERSECTION D'OUVERTS STRICTEMENT PSEUDOCONVEXES
}

\author{
Chantal Menini
}

\begin{abstract}
On a finite intersection of strictly pseudoconvex domains we define two kinds of natural Nevanlinna classes in order to take the growth of the functions near the sides or the edges into account. We give a sufficient Blaschke type condition on an analytic set for being the zero set of a function in a given Nevanlinna class. On the other hand we show that the usual Blaschke condition is not necessary here.
\end{abstract}

\section{Introduction}

La croissance d'une fonction holomorphe est liée à la répartition de ses zéros. Dans cet article, nous nous interessons à ce lien pour des fonctions de classes de Nevanlinna mais commençons par rappeler quelques résultats concernant ces classes.

Une fonction holomorphe sur $\Omega$, ouvert de $\mathbb{C}^{n}$ à frontière régulière, est dite dans la classe de Nevanlinna $N(\Omega)$ si

$$
\sup _{\varepsilon>0} \int_{\partial \Omega_{\varepsilon}} \ln ^{+}|f| d S_{\varepsilon}<+\infty
$$

où $\Omega_{\varepsilon}$ est l'ensemble des points de $\Omega$ à la distance au moins $\varepsilon$ du bord de $\Omega$.

Si $F$ est dans $N(\Omega)$ alors le courant d'intégration sur l'ensemble des zéros $X=\{F=0\}, \Theta_{X}=\frac{i}{\pi} \partial \bar{\partial} \ln |F|$, satisfait la condition de Blaschke

$$
\int_{\Omega} \delta \Theta_{X} \wedge \frac{\beta^{n-1}}{(n-1) !}<+\infty
$$

$\operatorname{avec} \delta(z)=\operatorname{dist}(z, \partial \Omega)$ et $\beta=\frac{i}{2} \partial \bar{\partial}|z|^{2}$. 
Henkin $[$ Hen] et Skoda [Sko] ont prouvé que lorsque l'ouvert $\Omega$ est strictement pseudoconvexe la réciproque est vraie. C'est à dire qu'étant donné un courant d'intégration $\Theta_{X}$ sur un ensemble analytique de dimension $n-1$ qui satisfait la condition de Blaschke, il existe une fonction holomorphe $F$ dans $N(\Omega)$ solution de $i \partial \bar{\partial} \ln |F|=\Theta_{X}$.

En fait, il suffit de trouver une solution plurisousharmonique $W$, à l'équation $i \partial \bar{\partial}$ car la résolution de $\ln |F|=W$ ne dépend que de conditions topologiques sur $\Omega$. Ainsi on généralise la notion de classe de Nevanlinna aux fonctions plurisousharmoniques et la condition de Blaschke aux $(1,1)$ courants positifs et fermés.

Une telle caractérisation pour $\Omega$ ouvert de type fini de $\mathbb{C}^{2}$ a été donnée par Chang, Nagel et Stein [C.N.S]. Pour $\mathbb{C}^{3}$, une première étape a été franchie par Charpentier et Dupain [C.D] avec l'estimation des coefficients tangents d'un courant positif et fermé.

Pour le bidisque la classe de Nevanlinna naturelle est définie par la condition

$$
\sup _{0<r<1} \int_{T^{2}} \ln ^{+}|f(r z)| d \sigma(z)<+\infty .
$$

Charpentier [Cha] et Andersson [And] ont donné des conditions suffisantes d'appartenance à cette classe et ce dernier a caractérisé des classes de Nevanlinna à poids dans le polydisque.

Dans notre étude nous prenons pour $\Omega$ un ouvert de $\mathbb{C}^{n}$ qui est l'intersection transverse de $N$ ouverts strictement pseudoconvexes, étoilés par rapport à 0 et à bords réguliers. Ils sont définis par :

$$
\Omega_{i}=\left\{z \in \mathbb{C}^{n} \mid \rho_{i}(z)<0\right\},
$$

avec $\rho_{i}$ fonction strictement plurisousharmonique et $\mathcal{C}^{2}$ ou $\mathcal{C}^{3}$ sur un voisinage de $\bar{\Omega}_{i}, d \rho_{i} \neq 0$ dans un voisinage de $\partial \Omega_{i}$.

Nous définissons sur $\Omega$ la fonction $\rho \operatorname{par} 1 / \rho=\sum_{i=1}^{N} 1 / \rho_{i}$, elle a la même régularité que les fonctions $\rho_{i}$; elle se comporte comme la distance à la frontière et est strictement plurisousharmonique sur $\Omega$. Ainsi, pour tout $\varepsilon>0$ les ouverts $\Omega_{\varepsilon}=\{z \in \Omega \mid \rho(z)<-\varepsilon\}$ sont strictement pseudoconvexes et de fonctions définissantes $\rho_{\varepsilon}=\rho+\varepsilon$.

Nous nous interessons à deux classes de Nevanlinna

Définition. Nous dirons qu'une fonction, $W$, plurisousharmonique sur $\Omega$, appartient à la classe de Nevanlinna
a) $N_{1}(\Omega)$ si $\left\|W^{+}\right\|_{N_{1}(\Omega, \rho)} \stackrel{\text { def }}{=} \sup _{\varepsilon>0} \int_{\partial \Omega_{\varepsilon}} W^{+} i \bar{\partial} \rho \wedge \beta^{n-1}<+\infty$,
b) $\quad N_{2}(\Omega)$ si $\left\|W^{+}\right\|_{N_{2}(\Omega, \rho)} \stackrel{\text { def }}{=} \sup _{\varepsilon>0} \int_{\partial \Omega_{\varepsilon}} W^{+} i \bar{\partial} \rho \wedge(i \partial \bar{\partial} \rho)^{n-1}<+\infty$. 
Le premier problème qui se pose est de savoir si, comme dans le cas strictement pseudoconvexe, le fait qu'une fonction soit dans $N_{1}(\Omega)$ implique que $\Theta=i \partial \bar{\partial} W$ satisfasse la condition de Blaschke; la réponse est non.

Le deuxième problème est de trouver des conditions suffisantes sur un $(1,1)$ courant $\Theta$ pour que l'équation $i \partial \bar{\partial} W=\Theta$ admette une solution dans l'une des classes de Nevanlinna.

Une condition suffisante pour qu'une solution soit dans la classe $N_{1}(\Omega)$ est

Théorème A. Si le $(1,1)$-courant positif et fermé $\Theta$ vérifie

$$
\int_{\Omega}-\rho i \partial \bar{\partial} \rho \wedge \Theta \wedge \beta^{n-2}<+\infty
$$

alors il existe une fonction plurisousharmonique $W$ dans la classe de Nevanlinna $N_{1}(\Omega)$, solution dans $\Omega$ de l'équation $i \partial \bar{\partial} W=\Theta$.

Pour qu'une solution du $i \partial \bar{\partial}$ soit dans la classe $N_{2}(\Omega)$, nous ne savons déterminer une condition suffisante que lorsque $\Omega$ est l'intersection de deux domaines

Théorème B. Supposons que le $(1,1)$-courant positif et fermé $\Theta$ vérifie

$$
\int_{\Omega}-\rho \Theta \wedge \beta^{n-1}<+\infty, \quad \int_{\Omega} i \partial \rho_{j} \wedge \bar{\partial} \rho_{j} \wedge \Theta \wedge \beta^{n-2}<+\infty(j=1,2),
$$

et pour $n \geq 3$

$$
\int_{\Omega}-\frac{1}{\rho}\left(\frac{\rho}{\rho_{1}}\right)^{3}\left(\frac{\rho}{\rho_{2}}\right)^{3} i \partial \rho_{1} \wedge \bar{\partial} \rho_{1} \wedge i \partial \rho_{2} \wedge \bar{\partial} \rho_{2} \wedge \Theta \wedge \beta^{n-3}<+\infty .
$$

Alors il existe une fonction plurisousharmonique $W$ dans la classe de Nevanlinna $N_{2}(\Omega)$, solution dans $\Omega$ de l'équation $i \partial \bar{\partial} W=\Theta$.

Pour résoudre l'équation $i \partial \bar{\partial} W=\Theta$ nous résolvons les équations $i d w=\Theta$ et $\bar{\partial} U=w_{0,1}$ où $w_{0,1}$ est la composante de bidegré $(0,1)$ de $w$. Alors $W=U+\bar{U}$ est solution de $i \partial \bar{\partial} W=\Theta$.

Nous résolvons le $\bar{\partial}$ avec les noyaux à poids de Berndtsson et Andersson [B.A], le choix du poids dépendant de la classe dans laquelle nous voulons trouver notre solution. 
Pour déterminer une solution du $i \partial \bar{\partial}$ dans $N_{1}(\Omega)$, nous résolvons $i d w=\Theta$ avec l'homotopie de Poincaré. Pour le cas de $N_{2}(\Omega)$, nous prenons une famille d'homotopies dépendant d'un paramètre $r$ et telles que pour tout $r \in[0,1], i d w_{r}=\Theta$. Ainsi, $w=\int_{0}^{1} w_{r} d r$ sera une solution de $i d w=\Theta$ satisfaisante pour notre problème.

Le reste du travail consiste à estimer "convenablement" ces solutions.

Le plan général est le suivant. Dans le paragraphe I nous vérifions quelques propriétés élémentaires satisfaites par $\rho$ et construisons un contre-exemple à ce que la condition de Blaschke soit nécéssaire. Une solution de $\bar{\partial} U=w_{0,1}$ est construite dans le paragraphe II et l'on en donne une estimation lorsque $w$ est de classe $\mathcal{C}^{1}$. Le paragraphe III consiste en la constrution d'une solution de $i d w=\Theta$ et son estimation lorsque $\Theta$ est de classe $\mathcal{C}^{2}$, puis après une régularisation nous établissons le Théorème A. Dans le paragraphe IV nous suivons les mêmes étapes que dans les paragraphes II et III et obtenons le Théorème B.

Ces résultats représentent la partie essentielle de la thèse de l'auteur [Men] où le lecteur pourra trouver plus de détails sur des estimations qui n'ont pas leur place ici.

Je remercie L. Gruman pour l'aide permanente qu'il m'a apportée lors de ces travaux.

\section{Préliminaires}

Nous nous donnons $N(N \geq 2)$ domaines $\Omega_{i}$ strictement pseudoconvexes de $\mathbb{C}^{n}$, étoilés par rapport à 0 , bornés et à frontière $\mathcal{C}^{2}$ régulière.

$\rho_{i}$ désignera la fonction définissante de $\Omega_{i}$ et sera $\mathcal{C}^{2}$ et strictement plurisousharmonique sur un voisinage de $\bar{\Omega}_{i}$. Posons

(1.3) $\forall I \subset\{1, \ldots, N\}, S_{I}=\left\{z \in \mathbb{C}^{n} \mid \rho_{i}(z)=0 \forall i \in I, \rho_{k}(z)<0 \forall k \notin I\right\}$,

$$
\begin{gathered}
\forall \delta>0, U_{i}^{\delta}=\left\{\rho_{i}(z)<\delta\right\}, V_{i}^{\delta}=\left\{-\delta<\rho_{i}(z)<\delta\right\}, \\
\tilde{\Omega}_{k}=\left\{z \in \Omega \mid-\rho_{k}(z)=\inf _{1 \leq i \leq N}-\rho_{i}(z)\right\} .
\end{gathered}
$$

L'intersection, $\Omega=\bigcap_{i=1}^{N} \Omega_{i}$, est supposée transverse, c'est à dire qu'il existe $\delta>0$ tel que pour tout $l \in\{1, \ldots, N\}$, pour toute suite $1 \leq i_{1}<$ $i_{2}<\ldots<i_{l} \leq N$, les 1 -formes $d \rho_{i_{1}}, d \rho_{i_{2}}, \ldots, d \rho_{i_{l}}$ sont $\mathbb{R}$-linéairement indépendantes en tout point de $V_{i_{1}}^{\delta} \cap V_{i_{2}}^{\delta} \cap \ldots \cap V_{i_{l}}^{\delta}$. 
Lemme 1.1. Soit $\rho$ la fonction définie en (1.1), alors

(1) $\rho$ est $\mathcal{C}^{2}$ et strictement pseudoconvexe sur $\Omega$. De plus, les valeurs propres de sa matrice Hessienne sont minorées sur $\Omega$ par une constante strictement positive.

(2) $\partial \rho=\sum_{j=1}^{N}\left(\frac{\rho}{\rho_{j}}\right)^{2} \partial \rho_{j}$

(3) $i \partial \bar{\partial} \rho-\frac{2}{\rho} i \partial \rho \wedge \bar{\partial} \rho=\sum_{j=1}^{N}\left(\left(\frac{\rho}{\rho_{j}}\right)^{2} i \partial \bar{\partial} \rho_{j}-\frac{2}{\rho_{j}}\left(\frac{\rho}{\rho_{j}}\right)^{2} i \partial \rho_{j} \wedge \bar{\partial} \rho_{j}\right)$.

(4) Il existe deux constantes strictement positives $C_{1}$ et $C_{2}$, telles que pour tout $z$ de $\Omega, C_{1} \operatorname{dist}(z, \partial \Omega) \leq-\rho(z) \leq C_{2} \operatorname{dist}(z, \partial \Omega)$.

Preuve:

(1) Pour $N=2, \rho=\frac{\rho_{1} \rho_{2}}{\rho_{1}+\rho_{2}}$ et en tant que courant $i \partial \bar{\partial} \rho$ est minoré par

$$
\left(\frac{\rho_{2}}{\rho_{1}+\rho_{2}}\right)^{2} i \partial \bar{\partial} \rho_{1}+\left(\frac{\rho_{1}}{\rho_{1}+\rho_{2}}\right)^{2} i \partial \bar{\partial} \rho_{2}
$$

qui est bien un $(1,1)$-courant strictement positif. Puis nous concluons par récurrence.

(2) et (3) s'obtiennent après un calcul immédiat.

(4) Il suffit de remarquer que sur $\Omega$,

$$
\frac{1}{N} \inf _{1 \leq i \leq N}\left(-\rho_{i}\right) \leq-\rho \leq \inf _{1 \leq i \leq N}\left(-\rho_{i}\right)
$$

et comme $-\rho_{i}$ est comparable à la distance à $\partial \Omega_{i}$, nous avons l'inégalité souhaitée.

Remarque. $\rho$ est "presque" une fonction définissante de $\Omega$ puisqu'elle est $\mathcal{C}^{2}$, plurisousharmonique sur $\Omega$ et se comporte comme la distance à la frontière. Ce serait une vraie fonction définissante si elle était définie sur un voisinage de $\bar{\Omega}$. Par contre, pour tout $\varepsilon>0, \rho_{\varepsilon}=\rho+\varepsilon$ est une fonction définissante pour $\Omega_{\varepsilon}$.

Définition 1.2. Nous dirons qu'une fonction, $W$, plurisousharmonique sur $\Omega$, appartient à la classe de Nevanlinna
a) $N_{1}(\Omega, \rho)$ si $\left\|W^{+}\right\|_{N_{1}(\Omega, \rho)} \stackrel{\text { def }}{=} \sup _{\varepsilon>0} \int_{\partial \Omega_{\varepsilon}} W^{+} i \bar{\partial} \rho \wedge \beta^{n-1}<+\infty$,
b) $N_{2}(\Omega, \rho)$ si $\left\|W^{+}\right\|_{N_{2}(\Omega, \rho)} \stackrel{\text { def }}{=} \sup _{\varepsilon>0} \int_{\partial \Omega_{\varepsilon}} W^{+} i \bar{\partial} \rho \wedge(i \partial \bar{\partial} \rho)^{n-1}<+\infty$, 
$\operatorname{avec} W^{+}=\sup (W, 0)$.

Remarque. $\rho$ étant $\mathcal{C}^{2}$, plurisousharmonique sur $\Omega$ et $d \rho \neq 0$ dans un voisinage de $\partial \Omega$, les $2 n-1$ formes volumes $\left.i \bar{\partial} \rho \wedge \beta^{n-1}\right|_{\partial \Omega_{\varepsilon}}$ et $\left.i \bar{\partial} \rho \wedge(i \partial \bar{\partial} \rho)^{n-1}\right|_{\partial \Omega_{\varepsilon}}$ induisent des mesures positives portées par $\partial \Omega_{\varepsilon}$ (cf. [Dem 1, Chap. 3]).

Lemme 1.3. Soit $\rho$ la fonction d'exhaustion définie par (1.1).

Soit $\tilde{\rho}: \Omega \rightarrow[-\infty, 0[$, une fonction plurisousharmonique, continue, exhaustive telle qu'il existe $\lambda_{1}, \lambda_{2}>0$ avec $\lambda_{1} \tilde{\rho} \leq \rho \leq \lambda_{2} \tilde{\rho}$ sur $\Omega$. Alors

$$
N_{1}(\Omega, \rho)=N_{1}(\Omega, \tilde{\rho}), \quad N_{2}(\Omega, \rho)=N_{2}(\Omega, \tilde{\rho})
$$

Nous noterons alors les classes de Nevanlinna $N_{1}(\Omega)$ ou $N_{2}(\Omega)$.

Preuve: Pour $N_{2}(\Omega)$ cela découle directement d'une preuve de J. P. Demailly [Dem 2, p. 528]. Pour $N_{1}(\Omega)$, nous la calquons en remplaçant la mesure $\mu_{\varphi, r}=\left.\left(d d^{c} \varphi\right)^{n-1} \wedge d^{c} \varphi\right|_{\{\varphi=r\}}$ par $m_{\varphi, r}=\bar{\partial} \varphi \wedge$ $\left.\beta^{n-1}\right|_{\{\varphi=r\}}$.

Remarque. On peut montrer que lorsque $W$ est dans $N_{1}(\Omega)$ la limite admissible de $W^{+}$est dans $L^{1}\left(S_{j}\right)(1 \leq j \leq N)$. Par contre la classe $N_{2}(\Omega)$ tient compte des "coins" du domaine, en particulier lorsque $\Omega=$ $\Omega_{1} \cap \Omega_{2}$ (seul cas où nous savons établir une condition suffisante), on peut montrer que si $W$ est dans $N_{2}(\Omega) W^{+}(t \cdot) i \bar{\partial} \rho_{1} \wedge i \bar{\partial} \rho_{2} \wedge \beta^{n-2}$ converge faiblement, lorsque $t$ croit vers 1 , vers une mesure bornée sur $S_{12}$.

Comme dans le cas strictement pseudoconvexe [Sko], [Hen], on peut penser que si une fonction plurisousharmonique $W$ est dans $N_{1}(\Omega)$ (la Classe de Nevanlinna la moins restrictive pour notre problème) alors $\Theta=i \partial \bar{\partial} W$ satisfait la Condition de Blaschke

$$
\int_{\Omega} \operatorname{dist}(\cdot, \partial \Omega) \Theta \wedge \beta^{n-1}<+\infty .
$$

Ce n'est plus vrai comme le contre-exemple suivant, pour l'intersection de deux boules dans $\mathbb{C}^{n}$, va nous le montrer.

Pour $p$ un entier fixé supérieur ou égal à deux, nous posons

$$
\begin{aligned}
& D_{1}=\left\{z \in \mathbb{C}|| z-\cos \frac{\pi}{2 p} \mid<1\right\}, \\
& D_{2}=\left\{z \in \mathbb{C}|| z+\cos \frac{\pi}{2 p} \mid<1\right\} .
\end{aligned}
$$


Lemme 1.4. Soit $D=D_{1} \cap D_{2}$, une transformation conforme $F$ de $D$ dans $U=\{z \in \mathbb{C}|| z \mid<1\}$ est donnée par

$$
\forall z \in D, \quad F(z)=i \frac{\left(z+i \sin \frac{\pi}{2 p}\right)^{p}-\left(i \sin \frac{\pi}{2 p}-z\right)^{p}}{\left(z+i \sin \frac{\pi}{2 p}\right)^{p}+\left(i \sin \frac{\pi}{2 p}-z\right)^{p}} .
$$

\section{Preuve:}

$1^{e}$ étape. $D$ est envoyé sur le secteur angulaire délimité par la demidroite $[0, x)$ et une demi-droite formant un angle de $\pi / p$ avec celle-ci, $Z_{1}=-e^{i \pi / 2 p} \frac{z+i \sin \frac{\pi}{2 p}}{z-i \sin \frac{\pi}{2 p}}$.

$2^{e}$ étape. Le secteur angulaire est envoyé sur le demi-plan supérieur, $Z_{2}=Z_{1}^{p}$.

$3^{e}$ étape. Le demi-plan supérieur est envoyé sur le disque unité, $Z_{3}=$ $i \frac{Z_{2}-i}{Z_{2}+i}=F(z)$.

Lemme 1.5. Posons pour tout entier $k$ supérieur à deux

$$
a_{k}^{p}=\sin \frac{\pi}{2 p}-\left(\frac{1}{k}\right)^{\frac{1}{p-1}} .
$$

Alors

(i) la fonction $f_{p}$ définie sur $D$ par

$$
f_{p}(z)=\prod_{k=2}^{\infty}\left(\frac{F\left(i a_{k}^{p}\right)-F(z)}{1-\bar{F}\left(i a_{k}^{p}\right) \cdot F(z)} \cdot \frac{\left|F\left(i a_{k}^{p}\right)\right|}{F\left(i a_{k}^{p}\right)}\right)
$$

est holomorphe et majorée en module par 1 sur $D$.

(ii) $\sum_{k \geq 2}\left(1-\left|i a_{k}^{p}-\cos \frac{\pi}{2 p}\right|^{2}\right)^{p-1}=+\infty$.

Preuve:

(i) D'après le Lemme $1.5, f_{p} \circ F^{-1}(z)$ est le produit de Blaschke sur $U$ qui a pour zéros $\left\{F\left(i a_{k}^{p}\right)\right\}_{k \geq 2}$, il suffit d'établir que $\sum_{k \geq 2}\left(1-\left|F\left(i a_{k}^{p}\right)\right|\right)<$ $+\infty$. Or

$$
\begin{aligned}
1-\left|F\left(i a_{k}^{p}\right)\right| & =2 \frac{(1 / k)^{\frac{p}{p-1}}}{\left(2 \sin \frac{\pi}{2 p}-\left(\frac{1}{k}\right)^{p-1}\right)^{p}+(1 / k)^{\frac{p}{p-1}}} \\
& \sim \frac{2}{\left(2 \sin \frac{\pi}{2 p}\right)^{p}}\left(\frac{1}{k}\right)^{\frac{p}{p-1}}
\end{aligned}
$$


qui est le terme général d'une série convergente.

(ii) $\left(1-\left|i a_{k}^{p}-\cos \frac{\pi}{2 p}\right|^{2}\right)^{p-1} \underset{k \uparrow \infty}{\sim}\left(2 \sin \frac{\pi}{2 p}\right)^{p-1} \frac{1}{k}$ qui est le terme général d'une série divergente.

Proposition 1.6. Dans $\mathbb{C}^{n}$ nous notons $z=\left(z_{1}, z^{\prime}\right)$ et définissons $\Omega=\Omega_{1} \cap \Omega_{2}$ avec

$$
\begin{aligned}
& \Omega_{1}=\left\{z|| z_{1}-\left.\cos \frac{\pi}{2(n+1)}\right|^{2}+\left|z^{\prime}\right|^{2}<1\right\}, \\
& \Omega_{2}=\left\{z|| z_{1}+\left.\cos \frac{\pi}{2(n+1)}\right|^{2}+\left|z^{\prime}\right|^{2}<1\right\} .
\end{aligned}
$$

Il existe une fonction $W$ dans la classe de Nevanlinna $N_{1}(\Omega)$ avec $i \partial \bar{\partial} W$ qui ne satisfait pas la condition de Blaschke.

Preuve: Posons pour tout $z$ de $\Omega, g(z)=f_{n+1}\left(z_{1}\right)$ où $f_{n+1}$ est la fonction définie par la formule (1.9). D'après le Lemme 1.5 (i), $W=\ln |g|$ est dans $N_{1}(\Omega)$ puisque $W^{+}=0$. Pour tout $z \operatorname{de} \Omega$, $\operatorname{dist}(z, \partial \Omega)$ est comparable à des constantes absolues près à inf $\left(1-\left|z_{1}-\cos \frac{\pi}{2(n+1)}\right|^{2}-\left|z^{\prime}\right|^{2}\right.$, $\left.1-\left|z_{1}+\cos \frac{\pi}{2(n+1)}\right|^{2}-\left|z^{\prime}\right|^{2}\right)$ et $\Delta_{z_{1}} \ln |f| d \lambda_{n}(z) \simeq \sum_{k \geq 2} \delta_{\left\{i a_{k}^{n+1}\right\}}\left(z_{1}\right)$ $d \lambda_{n-1}\left(z^{\prime}\right)$ avec $\delta_{\left\{i a_{k}^{n+1}\right\}}$ la masse de Dirac au point $i a_{k}^{n+1}$. Posons $D\left(i a_{k}^{n+1}\right)=\left\{\left.z^{\prime} \in \mathbb{C}^{n-1}|| z^{\prime}\right|^{2}<1-\left|i a_{k}^{n+1}-\cos \frac{\pi}{2(n+1)}\right|^{2}\right\}$, alors

$$
\begin{array}{r}
\sum_{k \geq 2} \int_{D\left(i a_{k}^{n+1}\right)}\left(1-\left|i a_{k}^{n+1}-\cos \frac{\pi}{2(n+1)}\right|^{2}-\left|z^{\prime}\right|^{2}\right) d \lambda_{n-1}\left(z^{\prime}\right) \\
\lesssim \int_{\Omega} \operatorname{dist}(\cdot, \partial \Omega) i \partial \bar{\partial} W \wedge \beta^{n-1}
\end{array}
$$

En intégrant par parties n-1 fois on obtient que l'intégrale de gauche est minorée à une constante absolue près par $\left(1-\left|i a_{k}^{n+1}-\cos \frac{\pi}{2(n+1)}\right|^{2}\right)^{n}$ et nous concluons avec le Lemme 1.5 (ii). 


\section{Résolution de l'équation $\bar{\partial} u=w$ et estimation sur les faces}

\subsection{Construction des solutions.}

Pour résoudre l'équation de Cauchy-Riemann nous utilisons les noyaux à poids de Berndtsson et Andersson $[\mathbf{B} . \mathbf{A}],[\mathbf{B e r}]$.

La stricte pseudoconvexité de chaque ouvert $\Omega_{i}$ permet d'après [For] de construire des fonctions support,

Théorème (FOR). Il existe $\delta>0$ tel que, pour tout $i$ de $\{1, \ldots, N\}$, $i l$ existe des fonctions $F^{(i)}(\zeta, z)$ vérifiant

(a) $F^{(i)} \in \mathcal{C}^{1}\left(U_{i}^{\delta} \times U_{i}^{\delta}\right)$,

(b) $\forall \zeta \in U_{i}^{\delta}, \quad F^{(i)}(\zeta, \cdot)$ holomorphe sur $U_{i}^{\delta}, F^{(i)}(\zeta, z)=\sum_{j=1}^{n}\left(\zeta_{j}-\right.$ $\left.z_{j}\right) F_{j}^{(i)}(\zeta, z), F_{j}^{(i)} \in \mathcal{C}^{1}\left(U_{i}^{\delta} \times U_{i}^{\delta}\right)$ holomorphe en $z$

(c) $\exists c_{i}>0, \forall z \in \bar{\Omega}, \forall \zeta \in \Omega_{i}, 2 \Re e F^{(i)}(\zeta, z) \geq \rho_{i}(\zeta)-\rho_{i}(z)+c_{i}|\zeta-z|^{2}$,

(d) $\left.d_{\zeta} F^{(i)}(\zeta, z)\right|_{z=\zeta}=\partial \rho_{i}(\zeta)$.

Pour construire les noyaux, nous reprenons les notations de $[\mathbf{B e r}$, p. 409] et posons

$$
\begin{aligned}
s_{k}(\zeta, z) & =\sum_{j=1}^{n}\left(-\rho_{k}(z)\left(\bar{\zeta}_{j}-\bar{z}_{j}\right)-\bar{F}^{(k)}(z, \zeta) F_{j}^{(k)}(z, \zeta)\right) d \zeta_{j} \\
Q^{(i)}(\zeta, z) & =\frac{1}{\rho_{i}(\zeta)} \sum_{j=1}^{n} F_{j}^{(i)}(\zeta, z) d \zeta_{j}, \quad(1 \leq i \leq N) \\
G(z)= & z^{-\alpha} \text { avec } \alpha>2, \\
G_{i}^{\left(\alpha_{i}\right)} & =G^{\left(\alpha_{i}\right)}\left(\left\langle Q^{(i)}(\zeta, z), z-\zeta\right\rangle+1\right) .
\end{aligned}
$$

Proposition-Définition 2.1. Soit $w$ une $(0,1)$-forme $\bar{\partial}$-fermée et $\grave{a}$ coefficients dans $\mathcal{C}^{1}(\bar{\Omega})$, une solution de $\bar{\partial} u=w$ est donnée par

(a) $\forall z \in \Omega, \quad u(z)=c_{n} \int_{\Omega} w(\zeta) \wedge K_{k}(\zeta, z) \quad \forall k \in\{1, \ldots, N\}$, avec

$$
\begin{aligned}
K_{k}= & \sum_{\substack{\left(\alpha_{0}, \ldots, \alpha_{N}\right) \\
\alpha_{0}+\cdots+\alpha_{N}=n-1}} \frac{(n-1) !}{\alpha_{1} ! \cdots \alpha_{N} !}\left(\prod_{i=1}^{N} G_{i}^{\left(\alpha_{i}\right)}\right) \\
& \frac{s_{k} \wedge\left(\bar{\partial} s_{k}\right)^{\alpha_{0}}}{\left\langle s_{k}, \zeta-z\right\rangle^{\alpha_{0}+1}} \bigwedge_{i=1}^{N}\left(\bar{\partial} Q^{(i)}\right)^{\alpha_{i}}
\end{aligned}
$$


(b) Pour tout $z$ de $S_{k}(1 \leq k \leq N), u(z)=c_{n} \int_{\Omega} w(\zeta) \wedge \tilde{K}_{k}(\zeta, z)$, avec

$$
\begin{aligned}
\tilde{K}_{k}(\zeta, z)= & \sum_{\substack{\left(\alpha_{1}, \ldots, \alpha_{N}\right) \\
\alpha_{1}+\cdots+\alpha_{N}=n-1}} \frac{(n-1) !}{\alpha_{1} ! \cdots \alpha_{N} !} \prod_{i=1}^{N} \frac{(-1)^{\alpha_{i}}\left(\alpha+\alpha_{i}\right) !\left(-\rho_{i}(\zeta)\right)^{\alpha+\alpha_{i}}}{\left(-\rho_{i}(\zeta)+F^{(i)}(\zeta, z)\right)^{\alpha+\alpha_{i}}} \\
& \times \frac{\sum_{l=1}^{n} F_{l}^{(k)}(z, \zeta) d \zeta_{l}}{-F^{(k)}(z, \zeta)} \bigwedge_{i=1}^{N}\left(\bar{\partial}_{\zeta} Q^{(i)}(\zeta, z)\right)^{\alpha_{i}}
\end{aligned}
$$

Preuve:

(a) Les conditions nécésaires pour pouvoir appliquer le Théorème 5 de [B.A] sont satisfaites. En effet $s_{k}, Q^{(i)}$ sont $\mathcal{C}^{1}$ sur $\Omega \times \Omega$ et

$$
|s(\zeta, z)| \leq C|\zeta-z|, \quad|\langle s(\zeta, z), \zeta-z\rangle| \geq c_{L}|\zeta-z|^{2},
$$

uniformément pour $\zeta$ dans $\bar{\Omega}, z$ dans $L$ compact de $\Omega$.

$Q^{(i)}(\zeta, \cdot)$ est holomorphe sur $\Omega$ pour tout $\zeta$ fixé dans $\Omega, \Re e\left(\left\langle Q^{(i)}(\zeta, z)\right.\right.$, $z-\zeta\rangle+1)>0$ sur $\Omega \times \bar{\Omega}$.

$G$ est holomorphe sur $\{\Re e(z)>0\}$ et $G(1)=1$.

D'après [B.A, Th. 5], nous avons pour tout $k$ de $\{1, \ldots, N\}$ la formule d'homotopie

$$
\begin{aligned}
w(z)=c_{1, n}\left\{-\int_{\partial \Omega} w(\zeta) \wedge K_{k}(\zeta, z)-\int_{\Omega}\right. & \bar{\partial} w(\zeta) \wedge K_{k}(\zeta, z) \\
& \left.+\bar{\partial}_{z} \int_{\Omega} w(\zeta) \wedge K_{k}(\zeta, z)\right\} .
\end{aligned}
$$

Le choix de $G$ avec $\alpha>2$ nous permet d'avoir $\prod_{i=1}^{N}\left(-\rho_{i}(\zeta)\right)$ en facteur dans les noyaux et donc $K_{k}(\zeta, z)=0 \forall(\zeta, z) \in \partial \Omega \times \Omega$. Ainsi nous avons une solution de $\bar{\partial} u=w$ pour toute $(0,1)$-forme $\bar{\partial}$-fermée qui est une fonction indépendante du choix des sections $s_{k}$, elle est donnée en (2.5).

(b) Pour tout $z \operatorname{sur} S_{k}, s_{k}(\zeta, z) \equiv-\bar{F}^{(k)}(z, \zeta) \sum_{i=1}^{n} F_{i}^{(k)}(z, \zeta) d \zeta_{i}$, or les fonctions $F_{i}^{(k)}(z, \cdot)$ sont holomorphes sur $\Omega$, d'où $s_{k} \wedge \bar{\partial}_{\zeta} s_{k} \equiv 0$ sur $\Omega \times S_{k}$. Grâce à la stricte pseudoconvexité, on peut montrer que $\int_{\Omega} w(\zeta) \wedge$ $K_{k}(\zeta, z)$ tend vers $\int_{\Omega} w(\zeta) \wedge \tilde{K}_{k}\left(\zeta, z_{0}\right)$ lorsque $z$ tend vers n'importe quel point $z_{0}$ de la face $S_{k}$. 


\subsection{Changement de variables.}

Pour estimer la solution du $\bar{\partial}$ nous allons utiliser des changements de variables analogues à ceux de Range et Siu $[$ R.S $]$.

Proposition-Définition 2.2. Pour tout $i$ de $\{1, \ldots, N\}$ on peut construire $p_{i}(\zeta, z)$ et $q_{i}(\zeta, z)$ des polynômes en $z$ de degré 2 , à coefficients fonctions de $\zeta$ uniformément bornées sur $\bar{\Omega}$, tels que

(1) $-\rho_{i}(z)=p_{i}(\zeta, z)+o\left(|\zeta-z|^{2}\right)$, $\Im m F^{(i)}(\zeta, z)=q_{i}(\zeta, z)+o\left(|\zeta-z|^{2}\right)$,

(2) $\frac{\partial p_{i}}{\partial z_{j}}(\zeta, z)=-\frac{\partial \rho_{i}}{\partial \zeta_{j}}(\zeta)+O(|\zeta-z|)$ $\frac{\partial q_{i}}{\partial z_{j}}(\zeta, z)=-\frac{1}{2 i} \frac{\partial \rho_{i}}{\partial \zeta_{j}}(\zeta)+O(|\zeta-z|)(1 \leq j \leq n)$,

(3) Il existe $\varepsilon_{0}>0$ tel que pour $|\zeta-z|<\varepsilon_{0},(\zeta, z) \in \bar{\Omega} \times \bar{\Omega}$,

$$
\begin{aligned}
& \left|-\rho_{i}(\zeta)+F^{(i)}(\zeta, z)\right| \gtrsim-\rho_{i}(\zeta)+\left|p_{i}(\zeta, z)\right|+\left|q_{i}(\zeta, z)\right|+|\zeta-z|^{2}, \\
& \forall z \in S_{i} \quad\left|F^{(i)}(z, \zeta)\right| \gtrsim-\rho_{i}(\zeta)+\left|q_{i}(\zeta, z)\right|+|\zeta-z|^{2} .
\end{aligned}
$$

\section{Preuve:}

$p_{i}(\zeta, z)$ et $q_{i}(\zeta, z)$ sont respectivement les développements de Taylor à l'ordre 2 de $-\rho_{i}(z)$ et $\Im m F^{(i)}(\zeta, z)$ entre $z$ et $\zeta$. Grâce à la régularité des fonctions $\rho_{i}$ et $F^{(i)}$ et au Théorème (FOR) (d), nous avons (1) et (2).

Avec le (c) du Théorème (FOR), nous avons pour tout $(\zeta, z) \in \bar{\Omega} \times \bar{\Omega}$

$$
-\rho_{i}(\zeta)+\Re e F^{(i)}(\zeta, z) \geq 1 / 2\left(-\rho_{i}(\zeta)-\rho_{i}(z)+c_{i}|\zeta-z|^{2}\right) .
$$

Le point (3) s'obtient avec (1), l'inégalité ci-dessus et

$$
\left|-\rho_{i}(\zeta)+F^{(i)}(\zeta, z)\right| \geq 1 / 2\left(\left|-\rho_{i}(\zeta)+\Re e F^{(i)}(\zeta, z)\right|+\left|\Im m F^{(i)}(\zeta, z)\right|\right) .
$$

Nous avons pour objectif de prendre pour $\zeta$ fixé, $p_{i}(\zeta, z)$ et $q_{i}(\zeta, z)$ comme nouvelles variables. Pour cela nous allons exprimer le numérateur de $\tilde{K}_{k}(\zeta, z)$ à l'aide de formes différentielles ne dépendant que des $d_{z} p_{i}$ et $d_{z} q_{i}$. Par raison de symétrie, il suffira de le faire pour $k=1$.

\subsection{Nouvelle expression du noyau.}

Au numérateur de $\tilde{K}_{1}(\zeta, z)$, nous avons la $(n, n)$-forme

$$
\sum_{l=1}^{n} F_{l}^{(1)}(z, \zeta) d \zeta_{l} \bigwedge_{i=1}^{N}\left(\bar{\partial}_{\zeta} Q^{(i)}(\zeta, z)\right)^{\alpha_{i}} \wedge w(\zeta)
$$


C'est surtout le module de cette forme qui nous intéresse et nous allons voir que, pour $\zeta$ fixé dans $\tilde{\Omega}_{k}$, si l'on ne peut pas mettre en facteur du module $\left|\bar{\partial} \rho_{k} \wedge w\right|$, alors le numérateur peut être transformé de sorte qu'il suffit de mettre $|w|$ en facteur. Pour toute $(p, q)$-forme $f=\sum_{\substack{|I|=p \\|J|=q}} f_{I, J}(\zeta) d \zeta_{I} \wedge d \bar{\zeta}_{J}$ nous notons $|f|=\sum_{\substack{|I|=p \\|J|=q}}\left|f_{I, J}(\zeta)\right|$.

Lemme 2.3. Soient $\zeta$ fixé dans $\tilde{\Omega}_{k} \cap V_{k}^{\delta}$ et I une partie de $\{1, \ldots, N\}$. Nous sommes nécessairement dans l'une des situations suivantes:

$$
\begin{aligned}
& k \in I \text { et }\left|\bigwedge_{i \in I} \bar{\partial} \rho_{i} \wedge w\right| \lesssim\left|\bar{\partial} \rho_{k} \wedge w\right| \bigwedge_{i \in I} \bar{\partial} \rho_{i} \mid \\
& k \notin I \text { et }\left|\bigwedge_{i \in I} \bar{\partial} \rho_{i} \wedge w\right| \lesssim|w|\left|\bar{\partial} \rho_{k} \bigwedge_{i \in I} \bar{\partial} \rho_{i}\right| \\
& k \notin I, \quad \exists i_{k} \in I \text { tel que } \\
& \left|\bigwedge_{i \in I} \bar{\partial} \rho_{i} \wedge w\right| \lesssim\left|\bar{\partial} \rho_{k} \wedge w\right|\left|\bar{\partial} \rho_{k} \bigwedge_{i \in I \backslash\left\{i_{k}\right\}} \bar{\partial} \rho_{i}\right| .
\end{aligned}
$$

Preuve: Il suffit de considérer le cas où $\bigwedge_{i \in I} \bar{\partial} \rho_{i}(\zeta) \wedge w(\zeta) \neq 0$.

- Si $k \in I$.

Via une rotation nous pouvons toujours nous ramener au cas où

$$
\begin{aligned}
\bar{\partial} \rho_{k}(\zeta)=a_{k} d \bar{\zeta}_{1} \text { avec } a_{k}=\frac{1}{2}\left|d \rho_{k}(\zeta)\right|, \quad w=\tilde{w}_{1} d \bar{\zeta}_{1}+\tilde{w}_{2} d \bar{\zeta}_{2} \\
\text { et } \bigwedge_{i \in I \backslash\{k\}} \bar{\partial} \rho_{i}=\sum_{\substack{J^{\prime} \\
1 \notin J^{\prime}}} \gamma_{J^{\prime}} d \bar{\zeta}_{J^{\prime}} .
\end{aligned}
$$

D'où

$$
\begin{aligned}
\left|\bigwedge_{i \in I} \bar{\partial} \rho_{i}(\zeta) \wedge w(\zeta)\right| & \leq a_{k}\left|\tilde{w}_{2}\right| \sum_{\substack{J^{\prime} \\
1,2 \notin J^{\prime}}}\left|\gamma_{J^{\prime}}(\zeta)\right| \\
& \leq \frac{2}{\left|d \rho_{k}(\zeta)\right|}\left|\bigwedge_{i \in I} \bar{\partial} \rho_{i}(\zeta)\right|\left|\bar{\partial} \rho_{k}(\zeta) \wedge w(\zeta)\right|
\end{aligned}
$$

Comme par hypothèse $\left|d \rho_{k}\right|$ est minoré par une constante strictement positive sur $V_{k}^{\delta}$, le point (1) est établi. 
- Si $k \notin I$.

Soit $\left(e_{1}, \ldots, e_{n}\right)$ une base orthonormée de l'espace tangent en un point $\zeta$ de $\Omega$, identifié à $\mathbb{C}^{n}$, et $\left(\gamma_{1}, \ldots, \gamma_{n}\right)$ sa base duale dans l'espace des $(0,1)$-formes. Quitte à faire une rotation, nous pouvons supposer que l'espace engendré par $\left(\gamma_{1}, \ldots, \gamma_{|I|+1}\right)$ est le même que celui engendré $\operatorname{par}\left\{\left(\bar{\partial} \rho_{i}\right)_{i \in I}, w\right\}$. Alors

$$
\bar{\partial} \rho_{k}(\zeta)=\sum_{i \in I} \alpha_{i} \bar{\partial} \rho_{i}(\zeta)+\alpha w(\zeta)+\bar{B}(\zeta)
$$

où $\bar{B}(\zeta)$ est dans l'espace engendré par $\left(\gamma_{|I|+2}, \ldots, \gamma_{n}\right)$.

$1^{e}$ cas. $|\bar{B}(\zeta)| \geq\left|\bar{\partial} \rho_{k}(\zeta)\right| / n$. Nous sommes dans le cas (2) puisque

$$
\left|\bar{\partial} \rho_{k}(\zeta) \bigwedge_{i \in I} \bar{\partial} \rho_{i}(\zeta) \wedge w(\zeta)\right|=|\bar{B}(\zeta)| \bigwedge_{i \in I} \bar{\partial} \rho_{i}(\zeta) \wedge w(\zeta) \mid .
$$

$2^{e}$ cas. $|\bar{B}(\zeta)|<\left|\bar{\partial} \rho_{k}(\zeta)\right| / n$.

$*$ Si $|\alpha| \geq \frac{1}{n} \frac{\left|\bar{\partial} \rho_{k}(\zeta)\right|}{|w(\zeta)|}$, alors

$$
\bar{\partial} \rho_{k}(\zeta) \bigwedge_{i \in I} \bar{\partial} \rho_{i}(\zeta)=\alpha w(\zeta) \bigwedge_{i \in I} \bar{\partial} \rho_{i}(\zeta)+\bar{B}(\zeta) \bigwedge_{i \in I} \bar{\partial} \rho_{i}(\zeta)
$$

Puisque $\bar{B}(\zeta)$ a été pris dans l'espace engendré par $\left(\gamma_{|I|+2}, \ldots, \gamma_{n}\right)$, nous avons

$$
|\alpha|\left|\bigwedge_{i \in I} \bar{\partial} \rho_{i}(\zeta) \wedge w(\zeta)\right| \leq\left|\bar{\partial} \rho_{k}(\zeta) \bigwedge_{i \in I} \bar{\partial} \rho_{i}(\zeta)\right|
$$

nous sommes encore dans le cas (2).

$*$ Si il existe $i_{k} \in I$ tel que $\left|\alpha_{i_{k}}\right| \geq \frac{1}{n} \frac{\left|\bar{\partial} \rho_{k}(\zeta)\right|}{\left|\bar{\partial} \rho_{i_{k}}(\zeta)\right|}$, alors

$$
\begin{aligned}
\bar{\partial} \rho_{k}(\zeta) \bigwedge_{i \in I \backslash\left\{i_{k}\right\}} & \bar{\partial} \rho_{i}(\zeta) \wedge w(\zeta) \\
& = \pm \alpha_{i_{k}} w(\zeta) \bigwedge_{i \in I} \bar{\partial} \rho_{i}(\zeta)+\bar{B}(\zeta) \bigwedge_{i \in I \backslash\left\{i_{k}\right\}} \bar{\partial} \rho_{i}(\zeta) \wedge w(\zeta)
\end{aligned}
$$

De même que pour le cas précédent

$$
\left|\bigwedge_{i \in I} \bar{\partial} \rho_{i}(\zeta) \wedge w(\zeta)\right| \lesssim\left|\bar{\partial} \rho_{k}(\zeta) \bigwedge_{i \in I \backslash\left\{i_{0}\right\}} \bar{\partial} \rho_{i}(\zeta) \wedge w(\zeta)\right|
$$

et en utilisant les mêmes majorations que pour le cas (1) nous obtenons (3). 
Lemme 2.4. Soient $(\zeta, z) \in \bar{\Omega} \times \bar{\Omega}$ tel que $|\zeta-z| \leq \varepsilon_{0}$, alors

$$
\begin{gathered}
\left|\left(\sum_{k=1}^{n} F_{k}^{(1)}(z, \zeta) d \zeta_{k}+\bar{F}_{k}^{(1)}(z, \zeta) d \bar{\zeta}_{k}\right) \bigwedge_{i \in I}\left(\sum_{k=1}^{n} F_{k}^{(i)}(\zeta, z) d \zeta_{k}\right) \bigwedge_{j \in J} \bar{\partial} \rho_{j}(\zeta)\right| \\
\quad \lesssim \sum_{\substack{I_{1}, I_{2}, I_{3} \\
J_{1}, J_{2}, J_{3}}}\left\{|\zeta-z|^{\left|I_{3}\right|+\left|J_{3}\right|}\left|d \rho_{1}(z) \bigwedge_{\substack{i \in I_{1} \\
\cup J_{1}}} d_{z} p_{i}(\zeta, z) \bigwedge_{\substack{j \in I_{2} \\
\cup J_{2}}} d_{z} q_{j}(\zeta, z)\right|\right. \\
\left.+|\zeta-z|^{1+\left|I_{3}\right|+\left|J_{3}\right|}\left|\bigwedge_{\substack{i \in I_{1} \\
\cup J_{1}}} d_{z} p_{i}(\zeta, z) \bigwedge_{\substack{j \in I_{2} \\
\cup J_{2}}} d_{z} q_{j}(\zeta, z)\right|\right\}
\end{gathered}
$$

avec $I_{1} \dot{\cup} I_{2} \dot{\cup} I_{3}=I, J_{1} \dot{\cup} J_{2} \dot{\cup} J_{3}=J$.

Preuve: Avec le Théorème (FOR) (d), nous avons pour tout $i$ de $\{1, \ldots, N\}$, tout $k$ de $\{1, \ldots, n\}$

$$
\left|F_{k}^{(i)}(\zeta, z)-\frac{\partial \rho_{i}}{\partial \zeta_{k}}(\zeta)\right| \lesssim|\zeta-z| .
$$

Avec la Proposition-Définition 2.2 (2), nous pouvons exprimer $\sum_{k=1}^{n} F_{k}^{(i)}(\zeta, z) d \zeta_{k}, \bar{\partial} \rho_{i}(\zeta)$ en fonction de formes faisant intervenir les dérivées de $p_{i}, q_{j}$ et celles majorées en module par $|\zeta-z|$. Puis nous terminons en utilisant la multilinéarité du produit extérieur et en remarquant que le module d'une forme est inchangé si l'on remplace les $d \zeta_{k}$, $d \bar{\zeta}_{k}$ par $d z_{k}, d \bar{z}_{k}$.

Lemme 2.5. Soient $\zeta$ fixé dans $\tilde{\Omega}_{k} \cap V_{k}^{\delta} \cap V_{1}^{\delta}$, I et $J$ deux parties de $\{1, \ldots, N\}$. Alors il existe $\eta_{0}>0$ et $z_{1}, \ldots, z_{p}$ dans $\bar{B}\left(\zeta, \varepsilon_{0}\right) \cap \bar{\Omega}$ satisfaisant

a) Pour $1 \leq r \leq p$, il existe $i_{r}$ dans $I$ (ou bien $j_{r}$ dans $J$ ) tel que pour tout $z$ de $B\left(z_{r}, \eta_{0}\right)$ on ait

$$
\left|\bigwedge_{i \in I} d_{z} p_{i}(\zeta, z) \bigwedge_{j \in J} d_{z} q_{j}(\zeta, z)\right| \lesssim\left|d \rho_{1}(z) \bigwedge_{i \in I \backslash\left\{i_{r}\right\}} d_{z} p_{i}(\zeta, z) \bigwedge_{j \in J} d_{z} q_{j}(\zeta, z)\right|
$$

ou bien

$$
\left|\bigwedge_{i \in I} d_{z} p_{i}(\zeta, z) \bigwedge_{j \in J} d_{z} q_{j}(\zeta, z)\right| \lesssim\left|d \rho_{1}(z) \bigwedge_{i \in I} d_{z} p_{i}(\zeta, z) \bigwedge_{j \in J \backslash\left\{j_{r}\right\}} d_{z} q_{j}(\zeta, z)\right| .
$$


b) $\operatorname{Sur} \bar{B}\left(\zeta, \varepsilon_{0}\right) \backslash \bigcup_{r=1}^{p} B\left(z_{r}, \eta_{0}\right)$

$\left|\bigwedge_{i \in I} d_{z} p_{i}(\zeta, z) \bigwedge_{j \in J} d_{z} q_{j}(\zeta, z)\right| \lesssim\left|d \rho_{1}(z) \bigwedge_{i \in I} d_{z} p_{i}(\zeta, z) \bigwedge_{j \in J} d_{z} q_{j}(\zeta, z)\right|$

Preuve: Nous pouvons supposer libre la famille $\left(d_{z} p_{i}, d_{z} q_{j}\right)_{\substack{i \in I \\ j \in J}}$

Considérons un point $z_{0}$ de $\bar{B}\left(\zeta, \varepsilon_{0}\right) \cap \bar{\Omega}$ tel $d \rho_{1}\left(z_{0}\right)$ appartienne à l'espace engendré par la famille ci-dessus. Comme dans le Lemme précédent nous remplaçons la forme $d_{z} p_{i}\left(\zeta, z_{0}\right)$ ou $d_{z} q_{j}\left(\zeta, z_{0}\right)$ la plus liée avec $d \rho_{1}\left(z_{0}\right)$ par ce dernier. Nous obtenons une inégalité du type a) avec $z=z_{0}$, par continuité en $z$ de ces formes différentielles, l'inégalité à une constante absolue près reste vraie sur $B\left(z_{0}, \eta_{z_{0}}\right)$ puis nous concluons grâce à la compacité de $\bar{B}\left(\zeta, \varepsilon_{0}\right)$.

Lemme 2.6. Soient $\zeta$ fixé dans $\tilde{\Omega}_{k_{1}} \cap V_{k_{1}}^{\delta} \cap V_{1}^{\delta}, z \in \bar{B}\left(\zeta, \varepsilon_{0}\right) \cap \bar{\Omega}$. Pour tout $N$-uplet $\left(\alpha_{1}, \ldots, \alpha_{N}\right)$ tel que $\alpha_{1}+\cdots+\alpha_{N}=n-1$, pour toute $(0,1)$-forme $w$,

$$
\left|\sum_{j=1}^{n} F_{j}^{(1)}(z, \zeta) d \zeta_{j} \bigwedge_{i=1}^{N}\left(\bar{\partial}_{\zeta} Q^{(i)}(\zeta, z)\right)^{\alpha_{i}} \wedge w(\zeta)\right|
$$

est majoré, à une constante absolue près, par la somme de termes du type suivant avec $(L, I)$ qui décrit l'ensemble des partitions de $\{1, \ldots, N\}$.

$* S i I=\emptyset$

$$
|w(\zeta)| \prod_{l=1}^{N}\left(-\frac{1}{\rho_{l}}(\zeta)\right)^{\alpha_{l}}
$$


* Si $k_{1} \in I$ et $\forall i \in I \quad \alpha_{i} \geq 1$

(2)

$$
\begin{aligned}
& \prod_{l \in L}\left(-\frac{1}{\rho_{l}}(\zeta)\right)^{\alpha_{l}} \prod_{i \in I}\left(-\frac{1}{\rho_{i}}(\zeta)\right)^{\alpha_{i}+1}\left|\bar{\partial} \rho_{k_{1}} \wedge w(\zeta)\right| \\
& \quad \times \sum_{\substack{I_{1} \cup I_{2} \cup I_{3} \\
\cup I_{4}=I}}|\zeta-z|^{\left|I_{2}\right|+\left|I_{3}\right|+2\left|I_{4}\right|}\left|d \rho_{1}(z) \bigwedge_{i \in I_{1} \cup I_{2}} d_{z} p_{i} \bigwedge_{j \in I_{1} \cup I_{3}} d_{z} q_{j}(\zeta, z)\right|
\end{aligned}
$$

(3)

$$
\begin{aligned}
& \prod_{l \in L}\left(-\frac{1}{\rho_{l}}(\zeta)\right)^{\alpha_{l}} \prod_{i \in I}\left(-\frac{1}{\rho_{i}}(\zeta)\right)^{\alpha_{i}+1}\left|\bar{\partial} \rho_{k_{1}} \wedge w(\zeta)\right| \\
& \quad \times \sum_{\substack{I_{1} \cup I_{2} \cup I_{3} \\
\cup I_{4}=I}}|\zeta-z|^{1+\left|I_{2}\right|+\left|I_{3}\right|+2\left|I_{4}\right|}\left|d \rho_{1}(z) \bigwedge_{i \in I_{1} \cup I_{2} \backslash\left\{i_{0}\right\}} d_{z} p_{i} \bigwedge_{j \in I_{1} \cup I_{3}} d_{z} q_{j}(\zeta, z)\right|
\end{aligned}
$$

où $\left(I_{1}, I_{2}, I_{3}, I_{4}\right)$ partition deI et $i_{0}$ dépendant de $z$ localement constant.

(3') Terme analogue en enlevant $i_{0} \grave{a} I_{1} \cup I_{3}$ au lieu de $I_{1} \cup I_{2}$.

* Si $k_{1} \notin I$ et $\forall i \in I \quad \alpha_{i} \geq 1$

(4)

$$
\begin{aligned}
& \prod_{l \in L}\left(-\frac{1}{\rho_{l}}(\zeta)\right)^{\alpha_{l}} \prod_{i \in I}\left(-\frac{1}{\rho_{i}}(\zeta)\right)^{\alpha_{i}+1}|w(\zeta)| \\
& \quad \times \sum_{\substack{I_{1} \cup I_{2} \cup I_{3}=I \\
J_{1} \cup J_{2} \cup J_{3}=I \cup\left\{k_{1}\right\}}}|\zeta-z|^{\left|I_{3}\right|+\left|J_{3}\right|}\left|d \rho_{1}(z) \bigwedge_{i \in I_{1} \cup J_{1}} d_{z} p_{i} \bigwedge_{j \in I_{2} \cup J_{2}} d_{z} q_{j}(\zeta, z)\right|
\end{aligned}
$$

(5)

$$
\begin{aligned}
& \prod_{l \in L}\left(-\frac{1}{\rho_{l}}(\zeta)\right)^{\alpha_{l}} \prod_{i \in I}\left(-\frac{1}{\rho_{i}}(\zeta)\right)^{\alpha_{i}+1}|w(\zeta)| \\
& \quad \times \sum_{\substack{I_{1} \cup I_{2} \cup I_{3}=I \\
J_{1} \cup J_{2} \cup J_{3}=I \cup\left\{k_{1}\right\}}}|\zeta-z|^{1+\left|I_{3}\right|+\left|J_{3}\right|}\left|d \rho_{1}(z) \bigwedge_{i \in I_{1} \cup J_{1} \backslash\left\{i_{0}\right\}} d_{z} p_{i} \bigwedge_{j \in I_{2} \cup J_{2}} d_{z} q_{j}(\zeta, z)\right|
\end{aligned}
$$

où $\left(I_{1}, I_{2}, I_{3}\right)$ partition de $I,\left(J_{1}, J_{2}, J_{3}\right)$ partition de $I \cup\{k\}$ et $i_{0}$ dépendant de z localement constant. 
(5') Terme analogue en enlevant $i_{0} \grave{a} I_{2} \cup J_{2}$ au lieu de $I_{1} \cup J_{1}$.

(6)

$$
\begin{aligned}
& \prod_{l \in L}\left(-\frac{1}{\rho_{l}}(\zeta)\right)^{\alpha_{l}} \prod_{i \in I}\left(-\frac{1}{\rho_{i}}(\zeta)\right)^{\alpha_{i}+1}\left|\bar{\partial} \rho_{k_{1}} \wedge w(\zeta)\right| \\
& \quad \times \sum_{\substack{I_{1} \cup I_{2} \cup I_{3}=I \\
J_{1} \cup J_{2} \cup J_{3}=I \cup\left\{k_{1}\right\} \backslash\left\{k_{0}\right\}}}|\zeta-z|^{\left|I_{3}\right|+\left|J_{3}\right|}\left|d \rho_{1}(z) \bigwedge_{i \in I_{1} \cup J_{1}} d_{z} p_{i} \bigwedge_{j \in I_{2} \cup J_{2}} d_{z} q_{j}(\zeta, z)\right|
\end{aligned}
$$

où $\left(I_{1}, I_{2}, I_{3}\right)$ partition de $I,\left(J_{1}, J_{2}, J_{3}\right)$ partition de $I \cup\left\{k_{1}\right\} \backslash\left\{k_{0}\right\}$ et $k_{0} \neq k_{1}$ dépendant de $k_{1}$ et $z$, localement constant.

(7)

$$
\begin{aligned}
& \prod_{l \in L}\left(-\frac{1}{\rho_{l}}(\zeta)\right)^{\alpha_{l}} \prod_{i \in I}\left(-\frac{1}{\rho_{i}}(\zeta)\right)^{\alpha_{i}+1}\left|\bar{\partial} \rho_{k_{1}} \wedge w(\zeta)\right| \\
& \quad \times \sum_{\substack{I_{1} \cup I_{2} \cup I_{3}=I \\
J_{1} \cup J_{2} \cup J_{3}=I \cup\left\{k_{1}\right\} \backslash\left\{k_{0}\right\}}}|\zeta-z|^{1+\left|I_{3}\right|+\left|J_{3}\right|} \mid \\
& d \rho_{1}(z) \bigwedge_{i \in I_{1} \cup J_{1} \backslash\left\{i_{0}\right\}} d_{z} p_{i} \bigwedge_{j \in I_{2} \cup J_{2}} d_{z} q_{j}(\zeta, z) \mid
\end{aligned}
$$

où $\left(I_{1}, I_{2}, I_{3}\right)$ partition de $I,\left(J_{1}, J_{2}, J_{3}\right)$ partition de $I \cup\left\{k_{1}\right\} \backslash\left\{k_{0}\right\}$ et $i_{0}$ dépendant de $z$ et localement constant.

(7') Terme analogue en enlevant $i_{0} \grave{a} I_{2} \cup J_{2}$ au lieu de $I_{1} \cup J_{1}$.

Preuve: Commençons par donner l'expression des $\left(\bar{\partial}_{\zeta} Q^{(i)}\right)^{\alpha_{i}}$ en fonction des $F_{j}^{(i)}$

$$
\begin{aligned}
\left(\bar{\partial}_{\zeta} Q^{(i)}\right)^{\alpha_{i}} & =\left(\frac{1}{-\rho_{i}(\zeta)}\right)^{\alpha_{i}}\left(\sum_{j, k=1}^{n} \frac{\partial F_{j}^{(i)}}{\partial \bar{\zeta}_{k}}(\zeta, z) d \zeta_{j} \wedge d \bar{\zeta}_{k}\right)^{\alpha_{i}} \\
+\alpha_{i}\left(\frac{1}{-\rho_{i}(\zeta)}\right)^{\alpha_{i}+1}\left(\sum_{j, k=1}^{n} \frac{\partial F_{j}^{(i)}}{\partial \bar{\zeta}_{k}}(\zeta, z) d \zeta_{j} \wedge d \bar{\zeta}_{k}\right)^{\alpha_{i}-1} & \wedge\left(\sum_{j=1}^{n} F_{j}^{(i)}(\zeta, z) d \zeta_{j}\right) \wedge \bar{\partial} \rho_{i}(\zeta)
\end{aligned}
$$

Puisque $F_{j}^{(i)}$ est $\mathcal{C}^{1}$ sur $\bar{\Omega} \times \bar{\Omega}$, nous majorons par une constante les 
termes de la forme $\sum_{j, k=1}^{n} \frac{\partial F_{j}^{(i)}}{\partial \bar{\zeta}_{k}}(\zeta, z) d \zeta_{j} \wedge d \bar{\zeta}_{k}$ et obtenons

$$
\begin{aligned}
& \left|\sum_{j=1}^{n} F_{j}^{(1)}(z, \zeta) d \zeta_{j} \bigwedge_{i=1}^{N}\left(\bar{\partial}_{\zeta} Q^{(i)}(\zeta, z)\right)^{\alpha_{i}} \wedge w(\zeta)\right| \\
& \leq|w(\zeta)| \prod_{l=1}^{N}\left(-\frac{1}{\rho_{l}}(\zeta)\right)^{\alpha_{l}}+\sum_{L, I} \prod_{l \in L}\left(-\frac{1}{\rho_{l}}(\zeta)\right)^{\alpha_{l}} \prod_{i \in I}\left(-\frac{1}{\rho_{i}}(\zeta)\right)^{\alpha_{i}+1} \\
& \quad \times\left|\sum_{j=1}^{n} F_{j}^{(1)}(z, \zeta) d \zeta_{j} \bigwedge_{i \in I}\left(\left(\sum_{j=1}^{n} F_{j}^{(i)}(\zeta, z) d \zeta_{j}\right) \wedge \bar{\partial} \rho_{i}(\zeta)\right) \wedge w(\zeta)\right|
\end{aligned}
$$

où $I \dot{U} L=\{1, \ldots, N\}$.

Le premier terme de la somme nous donne le terme (1).

Les autres sont obtenus en remarquant que

$$
\begin{aligned}
& \left|\sum_{j=1}^{n} F_{j}^{(1)}(z, \zeta) d \zeta_{j} \bigwedge_{i \in I}\left(\sum_{j=1}^{n} F_{j}^{(i)}(\zeta, z) d \zeta_{j}\right) \wedge \bar{\partial} \rho_{i}(\zeta) \wedge w(\zeta)\right| \\
= & \left|\sum_{j=1}^{n} F_{j}^{(1)}(z, \zeta) d \zeta_{j} \bigwedge_{i \in I}\left(\sum_{j=1}^{n} F_{j}^{(i)}(\zeta, z) d \zeta_{j}\right)\right|\left|\bigwedge_{i \in I} \bar{\partial} \rho_{i}(\zeta) \wedge w(\zeta)\right| .
\end{aligned}
$$

Puis nous appliquons le Lemme 2.3 à $\bigwedge_{i \in I} \bar{\partial} \rho_{i}(\zeta) \wedge w(\zeta)$.

Ensuite en remarquant que pour toute $(0, p)$-forme $\omega_{p}$

$$
\begin{aligned}
& \left|\sum_{j=1}^{n} F_{j}^{(1)}(z, \zeta) d \zeta_{j} \bigwedge_{i \in I}\left(\sum_{j=1}^{n} F_{j}^{(i)}(\zeta, z) d \zeta_{j}\right)\right|\left|\omega_{p}\right| \\
\leq & \left|\sum_{j=1}^{n}\left(F_{j}^{(1)}(z, \zeta) d \zeta_{j}+\bar{F}_{j}^{(1)}(z, \zeta) d \bar{\zeta}_{j}\right) \bigwedge_{i \in I}\left(\sum_{j=1}^{n} F_{j}^{(i)}(\zeta, z) d \zeta_{j}\right) \wedge \omega_{p}\right|,
\end{aligned}
$$

nous nous ramenons aux conditions d'application du Lemme 2.4 et terminons avec le Lemme 2.5.

Remarque. La nouvelle expression du numérateur nous indique les changements de variables "à faire" afin d'obtenir de bonnes estimations. Comme nous avons pris soin de faire apparaitre en facteur $d \rho_{1}(z)$, nous pourrons les restreindre à $z$ sur $S_{1}$. Ainsi "moralement" pour chaque indice $i \in I$ pour lequel nous perdons un $-\rho_{i}(\zeta)$ de plus comparé à 
$-\rho_{l}(\zeta), l \in L$, nous avons ainsi la possibilité de gagner du $|\zeta-z|^{2}$ avec soit deux changements de variables supplémentaires $\left(d_{z} p_{i}\right.$ et $\left.d_{z} q_{i}\right)$, soit un changement de variable supplémentaire $\left(d_{z} p_{i}\right.$ ou $\left.d_{z} q_{i}\right)$ associé à du $|\zeta-z|$, soit avec du $|\zeta-z|^{2}$ en facteur.

Ce qui suit ne fait que vérifier par le calcul cette remarque.

\subsection{Estimations.}

Théorème 2.7. Pour toute $(0,1)$-forme $w, \bar{\partial}$-fermée sur $\Omega$, à coefficients dans $\mathcal{C}^{1}(\bar{\Omega})$, la solution de $\bar{\partial} u=w$ définie par la PropositionDéfinition 2.1 vérifie pour tout $j$ de $\{1, \ldots N\}$

$\int_{S_{j}}|u(z)| d \sigma_{j}(z) \lesssim \int_{\Omega}|w(\zeta)| d \lambda(\zeta)+\sum_{k=1}^{N} \int_{\tilde{\Omega}_{k}}\left(-\frac{1}{\rho_{k}}\right)^{1 / 2}\left|\bar{\partial} \rho_{k} \wedge w(\zeta)\right| d \lambda(\zeta)$.

Preuve: Nous commençons par découper $\Omega$ en l'union des $\tilde{\Omega}_{k}$ puis avec l'aide du Théorème de Fubini nous intégrons sur $S_{j}$ avec $\zeta$ fixé dans $\tilde{\Omega}_{k}$. Les seules majorations qui ne sont pas immédiates sont celles pour lesquelles $z \in S_{i} \cap B\left(\zeta, \varepsilon_{0}\right)$ et $\zeta \in \tilde{\Omega}_{k} \cap V_{k}^{\delta} \cap V_{i}^{\delta}$. Nous allons traiter le cas $i=1$ et avec le Lemme 2.6 nous sommes en mesure, pour tout $z$ de $V_{i}^{\delta} \cap B\left(\zeta, \varepsilon_{0}\right)$, de majorer

$$
\left|\sum_{j=1}^{n} F_{j}^{(1)}(z, \zeta) d \zeta_{j} \bigwedge_{i=1}^{N}\left(\bar{\partial}_{\zeta} Q^{(i)}(\zeta, z)\right)^{\alpha_{i}} \wedge w(\zeta)\right|
$$

par une somme de modules de formes différentielles toutes liées à $d \rho_{1}$. Pour toute forme différentielle $w$ définie sur un voisinage de $\bar{\Omega}, \mid\left(d \rho_{1} \wedge\right.$ $w)_{\mid S_{1}} \mid$ est égal, à une constante absolue près, à $\left|\varphi_{1}^{*}(w)\right|$ où $\varphi_{1}$ est la restriction à $S_{1}$, ce qui nous permet d'effectuer les estimations sur la face $S_{1}$.

Pour cela nous allons prendre comme nouvelles variables les $p_{i}(\zeta, z)$ et $q_{j}(\zeta, z)$ qui sont des polynômes en $z$ à coefficients localement bornés. Pour $\varepsilon_{0}$ assez petit, nous pouvons choisir les coordonnées réelles $x=$ $\left(x_{1}, \ldots, x_{2 n-1}, \rho_{1}\right)$ sur $B\left(\zeta, \varepsilon_{0}\right)$ de sorte que $x^{\prime}=\left(x_{1}, \ldots, x_{2 n-1}\right)$ soient les coordonnées réelles sur $S_{1} \cap B\left(\zeta, \varepsilon_{0}\right)$. Les ensembles

$$
\begin{aligned}
\left\{\left(x^{\prime}, p_{i}, q_{j}\right) \in \mathbb{R}^{2 n-1+|I|+|J|} \mid p_{i}\right. & =p_{i}\left(\zeta, x^{\prime}, 0\right), \forall i \in I ; \\
q_{j} & \left.=q_{j}\left(\zeta, x^{\prime}, 0\right), \forall j \in J\right\}
\end{aligned}
$$

où $I, J$ sont inclus dans $\{1, \ldots, N\}$, forment des recouvrements ramifiés finis de notre domaine d'intégration. Ainsi après changement de variables, nous obtiendrons une majoration à une constante absolue près de notre intégrale de départ.

Pour les majorations nous utiliserons systématiquement le 


\section{Lemme 2.8.}

Pour $I^{\prime} \dot{\cup} J^{\prime} \dot{\cup} K^{\prime} \subset\{1, \ldots, N\}, \beta_{i}>2 \forall i \in I^{\prime}, \beta_{j}>1 \forall j \in J^{\prime} \cup K^{\prime}$, $a_{l}>0 \forall l \in I^{\prime} \cup J^{\prime} \cup K^{\prime}$ et $R>0$ donnés, nous avons

(1)

$$
\begin{array}{r}
\prod_{i \in I^{\prime}} \int_{[0, R]^{2}} \frac{d p_{i} d q_{i}}{\left(a_{i}+p_{i}+q_{i}\right)^{\beta_{i}}} \prod_{j \in J^{\prime}} \int_{[0, R]} \frac{d p_{j}}{\left(a_{j}+p_{j}\right)^{\beta_{j}}} \prod_{k \in K^{\prime}} \int_{[0, R]} \frac{d q_{k}}{\left(a_{k}+q_{k}\right)^{\beta_{k}}} \\
\lesssim \prod_{i \in I^{\prime}}\left(\frac{1}{a_{i}}\right)^{\beta_{i}-2} \prod_{j \in I^{\prime} \cup K^{\prime}}\left(\frac{1}{a_{j}}\right)^{\beta_{j}-1} .
\end{array}
$$

Pour $a>0, \beta>0$, nous avons

$$
\text { (2) } \int_{[0, R]} \frac{r d r}{\left(a+r^{2}\right)^{\beta+1}} \lesssim \frac{1}{a^{\beta}}, \quad \text { (3) } \quad \int_{[0, R]} \frac{d r}{\left(a+r^{2}\right)^{\beta+1}} \lesssim \frac{1}{a^{\beta+1 / 2}} \text {. }
$$

Dans toute la suite nous supposerons $\zeta$ fixé dans $\tilde{\Omega}_{k_{1}} \cap V_{k_{1}}^{\delta} \cap V_{1}^{\delta}$ et allons estimer les intégrales sur $S_{1}$ qui proviennent du Lemme 2.6, nous notons $T_{i}(\zeta)$ l'intégrale sur $S_{1} \cap B\left(\zeta, \varepsilon_{0}\right)$ du terme (i).

$$
T_{1}(\zeta)=\int_{S_{1} \cap B\left(\zeta, \varepsilon_{0}\right)}\left(\prod_{l=1}^{N} \frac{\left(-\rho_{l}(\zeta)\right)^{\alpha}}{\left|-\rho_{l}(\zeta)+F^{(l)}(\zeta, z)\right|^{\alpha+\alpha_{l}}}\right) \frac{d \sigma_{1}(z)}{\left|F^{(1)}(z, \zeta)\right|} .
$$

Puisque $d \rho_{1} \neq 0$ dans un voisinage de $S_{1}$ nous pouvons prendre comme nouvelle variable $q_{1}=q_{1}(\zeta, z)$ et compléter avec les coordonnées manquantes. L'image de $S_{1} \cap B\left(\zeta, \varepsilon_{0}\right)$ par ce changement peut être mis dans un cube de $\mathbb{R}^{2 n-1},[-R, R]^{2 n-1}$. Puis après un passage en polaires, nous obtenons

$$
T_{1}(\zeta) \lesssim|w(\zeta)| \int_{[0, R]^{2}} \prod_{l=2}^{N} \frac{\left(-\rho_{l}\right)^{\alpha}}{\left(-\rho_{l}+r^{2}\right)^{\alpha+\alpha_{l}}} \frac{\left(-\rho_{1}\right)^{\alpha} r^{2 n-3} d r d q_{1}}{\left(-\rho_{1}+r^{2}+q_{1}\right)^{\alpha+\alpha_{1}+1}} .
$$

Sachant que $\zeta \in \tilde{\Omega}_{k_{1}}$ et que $\sum_{i=1}^{N} \alpha_{i}=n-1$, nous avons avec les inégalités (1) et (2) du Lemme 2.8

$$
T_{1}(\zeta) \lesssim|w(\zeta)| .
$$

Majorons maintenant l'intégrale type pour nos estimations

$$
\begin{aligned}
& T_{2}(\zeta)=\left|\bar{\partial} \rho_{k_{1}} \wedge w(\zeta)\right| \int_{\substack{S_{1} \cap \\
B\left(\zeta, \varepsilon_{0}\right)}} \frac{|\zeta-z|^{\left|I_{2}\right|+\left|I_{3}\right|+2\left|I_{4}\right|}}{\left|F^{(1)}(z, \zeta)\right|} \prod_{l \in L} \frac{\left(-\rho_{l}(\zeta)\right)^{\alpha}}{\left|-\rho_{l}(\zeta)+F^{(l)}(\zeta, z)\right|^{\alpha+\alpha_{l}}} \\
& \times \prod_{i \in I} \frac{\left(-\rho_{i}(\zeta)\right)^{\alpha-1}}{\left|-\rho_{i}(\zeta)+F^{(i)}(\zeta, z)\right|^{\alpha+\alpha_{i}}}\left|\bigwedge_{i \in I_{1} \cup I_{2}} d_{z} p_{i} \bigwedge_{j \in I_{1} \cup I_{3}} d_{z} q_{j}(\zeta, z)\right| d \sigma_{1}(z),
\end{aligned}
$$


avec les conventions habituelles, $L=\{1, \ldots, N\} \backslash I, \forall i \in I$ $\left(=I_{1} \dot{\cup} I_{2} \dot{\cup} I_{3} \dot{\cup} I_{4}\right) \alpha_{i} \geq 1$.

Pour majorer $T_{2}$ nous faisons les changements de variables suivants

$$
\forall i \in I_{1} \cup I_{2}, \quad p_{i}=p_{i}(\zeta, z) ; \quad \forall j \in I_{1} \cup I_{3}, \quad q_{j}=q_{j}(\zeta, z) .
$$

Ces nouvelles variables sont au plus au nombre de $2|I|$ qui est majoré par $2(n-1)$. Nous complétons avec des coordonnées qui sont libres avec les nouvelles variables ci-dessus (sinon l'intégrale correspondante est nulle). Après un passage en polaires, puisqu'au numérateur de notre intégrale apparait le Jacobien du changement de variables, nous avons la majoration

$$
\begin{aligned}
& T_{2}(\zeta) \lesssim\left|\bar{\partial} \rho_{k_{1}} \wedge w(\zeta)\right| \int_{[0, R]} \prod_{l \in L} \frac{\left(-\rho_{l}\right)^{\alpha}}{\left(-\rho_{l}+r^{2}\right)^{\alpha+\alpha_{l}}} \prod_{s \in I_{4}} \frac{\left(-\rho_{s}\right)^{\alpha-1} r^{2}}{\left(-\rho_{s}+r^{2}\right)^{\alpha+\alpha_{s}}} \\
& \times \frac{r^{2\left(n-1-\left|I_{1}\right|\right)}}{-\rho_{1}+r^{2}} d r\left\{\prod_{i \in I_{1}} \int_{[0, R]^{2}} \frac{\left(-\rho_{i}\right)^{\alpha-1} d p_{i} d q_{i}}{\left(-\rho_{i}+r^{2}+p_{i}+q_{i}\right)^{\alpha+\alpha_{i}}}\right. \\
&\left.\prod_{j \in I_{2}} \int_{[0, R]} \frac{\left(-\rho_{j}\right)^{\alpha-1} d p_{j}}{\left(-\rho_{j}+r^{2}+p_{j}\right)^{\alpha+\alpha_{j}}} \prod_{k \in I_{3}} \int_{[0, R]} \frac{\left(-\rho_{k}\right)^{\alpha-1} d q_{k}}{\left(-\rho_{k}+r^{2}+q_{k}\right)^{\alpha+\alpha_{k}}}\right\} .
\end{aligned}
$$

Puisque $\zeta \in \tilde{\Omega}_{k_{1}}$ et $\sum_{i=1}^{N} \alpha_{i}=n-1$, avec les inégalités (1) et (3) du Lemme 2.8 nous avons

$$
\begin{aligned}
T_{2}(\zeta) & \lesssim\left|\bar{\partial} \rho_{k_{1}} \wedge w(\zeta)\right| \int_{[0, R]} \frac{\left(-\rho_{k_{1}}\right)^{\alpha-1} r^{2\left(n-1-\left|I_{1}\right|\right)} d r}{\left(-\rho_{k_{1}}+r^{2}\right)^{\alpha+n-1-\left|I_{1}\right|}} \\
& \lesssim \frac{1}{\sqrt{-\rho_{k_{1}}}}\left|\bar{\partial} \rho_{k_{1}} \wedge w(\zeta)\right|
\end{aligned}
$$

Si l'on compare le terme (3) (ou (3')) à (2), on constate que l'on a perdu la possibilité de faire un changement de variable $\left(p_{i_{0}}\right.$ pour $(3), q_{i_{0}}$ pour $\left.\left(3^{\prime}\right)\right)$ mais qu'en revanche on a gagné une puissance de $|\zeta-z|$. Or en regardant les estimations ci-dessus on constate que l'effet est le même, c'est à dire le gain d'une demi-puissance pour le facteur $\left|-\rho_{i_{0}}+F^{\left(i_{0}\right)}(\zeta, z)\right|^{\alpha+\alpha_{i_{0}}} \mathrm{du}$ dénominateur. Ainsi

$$
T_{3}(\zeta), T_{3^{\prime}}(\zeta) \lesssim \frac{1}{\sqrt{-\rho_{k_{1}}}}\left|\bar{\partial} \rho_{k_{1}} \wedge w(\zeta)\right| .
$$

Le terme (4) (resp. (5)) donne la possibilité de faire un changement de variable supplémentaire avec $q_{k_{1}}$ par rapport au terme (2) (resp. (3)). 
Cela permet de gagner du $\left(-\rho_{k_{1}}(\zeta)\right)^{1 / 2}$ qui, par définition de $\tilde{\Omega}_{k_{1}}$, est inférieur à tout $\left(-\rho_{i}(\zeta)\right)^{1 / 2}, 1 \leq i \leq N$. Ainsi

$$
T_{4}(\zeta), T_{5}(\zeta), T_{5^{\prime}}(\zeta) \lesssim|w(\zeta)| .
$$

Dans le terme (6) (resp. (7)) par rapport à (2) (resp. (3)) la possibilité de changement de variable en $q_{k_{0}}$ est remplacée par celle en $q_{k_{1}}$ ce qui, pour l'estimation, est sans conséquence toujours grâce au fait que $\zeta$ est dans $\tilde{\Omega}_{k_{1}}$. D'où

$$
T_{6}(\zeta), T_{7}(\zeta), T_{7^{\prime}}(\zeta) \lesssim \frac{1}{\sqrt{-\rho_{k_{1}}}}\left|\bar{\partial} \rho_{k_{1}} \wedge w(\zeta)\right| .
$$

Le Théorème 2.7 est établi avec les majorations (2.7) à (2.11)

\section{Condition suffisante d'appartenance à la classe de nevanlinna $N_{1}(\Omega)$}

L'objet de ce paragraphe est d'établir le

Théorème 3.1. Si le $(1,1)$-courant positif et fermé $\Theta$ vérifie

$$
\int_{\Omega}-\rho i \partial \bar{\partial} \rho \wedge \Theta \wedge \beta^{n-2}<+\infty
$$

alors il existe une fonction plurisousharmonique $W$ dans la classe de Nevanlinna $N_{1}(\Omega)$, solution dans $\Omega$ de l'équation $i \partial \bar{\partial} W=\Theta$.

Nous commencerons par établir ce Théorème lorsque $\Theta$ est à coefficients $\mathcal{C}^{2}$ sur un voisinage de $\bar{\Omega}$, puis nous obtiendrons le cas général après régularisation.

Proposition 3.2. Soit $\Theta$ un $(1,1)$-courant positif et fermé sur $\Omega$. S'il vérifie la condition du Théorème 3.1, alors

(1) $B_{0}(\Theta)=\int_{\Omega}-\rho \Theta \wedge \beta^{n-1}<+\infty$

(2) $B_{j}(\Theta)=\int_{\Omega}\left(\frac{\rho}{\rho_{j}}\right)^{3} i \partial \rho_{j} \wedge \bar{\partial} \rho_{j} \wedge \Theta \wedge \beta^{n-2}<+\infty, \quad \forall j \in\{1, \ldots, N\}$.

Preuve: (1) Nous avons vu dans le Lemme 1.1 (1) qu'en tant que $(1,1)$ courant $i \partial \bar{\partial} \rho$ est minoré sur $\Omega$ par $C \beta$ avec $C>0$. Puisque $\Theta$ est un 
courant positif, $-\rho i \partial \bar{\partial} \rho \wedge \Theta \wedge \beta^{n-2}$ est minoré par $C(-\rho) \Theta \wedge \beta^{n-1}$ et l'inégalité (1) résulte directement des hypothèses du Théorème 3.1.

(2) Supposons $\Theta$ à coefficients $\mathcal{C}^{1}$ sur $\Omega, \Theta$ étant fermé avec la formule de Stokes appliquée à $(-\rho-\varepsilon) i \bar{\partial} \rho \wedge \Theta \wedge \beta^{n-2}$ sur $\Omega_{\varepsilon}$ et après avoir fait décroitre $\varepsilon$ vers 0 , nous avons

$$
\int_{\Omega} i \partial \rho \wedge \bar{\partial} \rho \wedge \Theta \wedge \beta^{n-2} \leq \int_{\Omega}-\rho i \partial \bar{\partial} \rho \wedge \Theta \wedge \beta^{n-2}
$$

Nous avions vu dans le Lemme 1.1 (3) que

$$
\begin{aligned}
& -\rho i \partial \bar{\partial} \rho+2 i \partial \rho \wedge \bar{\partial} \rho \\
& =\sum_{j=1}^{N}\left(-\rho\left(\frac{\rho}{\rho_{j}}\right)^{2} i \partial \bar{\partial} \rho_{j}+2\left(\frac{\rho}{\rho_{j}}\right)^{3} i \partial \rho_{j} \wedge \bar{\partial} \rho_{j}\right) .
\end{aligned}
$$

Comme pour tout $j$ de $\{1, \ldots, N\}, i \partial \bar{\partial} \rho_{j} \wedge \Theta \wedge \beta^{n-2}$ et $i \partial \rho_{j} \wedge \bar{\partial} \rho_{j} \wedge \Theta \wedge$ $\beta^{n-2}$ sont des mesures positives, l'inégalité (2) découle de (3.1) et (3.2).

Lorsque $\Theta$ n'est plus à coefficients $\mathcal{C}^{1}$, nous nous y ramenons par régularisation puis nous terminons par un passage à la limite standard.

\subsection{Résolution dans le cas $\mathcal{C}^{2}$.}

$\Theta$ est supposé à coefficients $\mathcal{C}^{2}$ sur $\bar{\Omega}$ et pour résoudre l'équation $i \partial \bar{\partial} W=\Theta$, nous suivons la méthode classique. Nous commençons par résoudre $i d w=\Theta$, puis nous décomposons $w=-w_{1,0}+w_{0,1}$ où $w_{1,0}$ et $w_{0,1}$ sont de bidegré $(1,0)$ et $(0,1)$. Comme le courant $\Theta$ est réel, nous pouvons prendre $w_{1,0}=\bar{w}_{0,1}$ et $w_{0,1}$ est $\bar{\partial}$-fermée. Alors une solution de $\bar{\partial} U=w_{0,1}$ vérifie $i \partial \bar{\partial}(U+\bar{U})=\Theta$.

Nous notons $\Theta=\sum_{j, k=1}^{n} \Theta_{j k} d z_{j} \wedge d \bar{z}_{k}$ et comme $\Omega$ est étoilé par rapport à 0 , nous construisons $w_{0,1}$ avec la formule d'homotopie

$$
w_{0,1}(z)=\sum_{j, k=1}^{n}\left(\int_{0}^{1} t z_{j} \Theta_{j k}(t z) d t\right) d \bar{z}_{k} .
$$

Comme solution de $\bar{\partial} U=w_{0,1}$, nous prendrons celle définie au paragraphe II dans la Proposition-Définition 2.1, mais auparavant il faut s'assurer que $w_{0,1}$ vérifie de "bonnes estimations".

Proposition 3.3. Soit $\Theta$ un $(1,1)$ courant positif et fermé, à coefficients dans $\mathcal{C}^{2}(\bar{\Omega})$. Soit $w_{0,1}$ la $(0,1)$-forme construite avec 
l'homotopie (3.3). Alors

(1) $\int_{\Omega}\left|w_{0,1}\right| d \lambda \lesssim B_{0}(\Theta)$,

(2) $\int_{\tilde{\Omega}_{k}}\left(-\frac{1}{\rho_{k}}\right)^{1 / 2}\left|\bar{\partial} \rho_{k} \wedge w_{0,1}\right| d \lambda \lesssim B_{0}(\Theta)+B_{k}(\Theta), \quad \forall k \in\{1, \ldots, N\}$.

Preuve: Nous calquons une méthode de Skoda [Sko].

Quitte à modifier nos solutions, sans pour autant changer leur comportement au bord de $\Omega$, nous pouvons supposer que $\Theta$ est identiquement nul sur $\bar{B}\left(0, c_{0}\right)$, avec $c_{0}$ choisi assez petit de sorte que $\bar{B}\left(0, c_{0}\right)$ soit inclus dans $\Omega$.

Pour tout $j \in\{1, \ldots, N\}, \Omega_{j}$ est par hypothèse étoilé par rapport à 0 , par conséquent, nous pouvons définir $J_{j}$ (resp. $J$ ) la jauge de $\Omega_{j}$ (resp. $\Omega)$. $\Omega$

Soit $\left.t_{0} \in\right] 0,1\left[\right.$ tel que $t_{0} \Omega \subset B\left(0, c_{0}\right)$, nous définissons pour tout $\zeta$ de

$$
t(\zeta)=\inf \left\{t \geq t_{0} \mid \zeta / t \in \Omega\right\} .
$$

Comme $t(\zeta)=\max \left(t_{0}, J(\zeta)\right)$ et $-\rho$ est comparable, à des constantes près, à $1-J$, pour tout $\zeta$ de $\Omega$

$$
\begin{aligned}
& \int_{t(\zeta)}^{1}(-\rho)^{-1 / 2}(\zeta / t) d t \lesssim(-\rho)^{1 / 2}(\zeta) \\
& \int_{t(\zeta)}^{1}(1-t)(-\rho)^{-1 / 2}(\zeta / t) d t \lesssim(-\rho)^{3 / 2}(\zeta) .
\end{aligned}
$$

Inégalité (1). Nous notons $\tau=2 \sum_{j=1}^{n} \Theta_{j j}$, la trace de $\Theta$. Comme c'est un $(1,1)$-courant positif, nous avons pour tout $\zeta$ de $\Omega$

$$
\left|\sum_{j, k=1}^{n} t \zeta_{j} \Theta_{j k}(t \zeta) d \bar{\zeta}_{k}\right| \lesssim \tau(t \zeta)
$$

Avec l'expression (3.3) de $w_{0,1}$, après changement de variable $\xi=t \zeta$ et l'application du Théorème de Fubini

$$
\begin{aligned}
\int_{\Omega}\left|w_{0,1}\right| d \lambda(\zeta) & \lesssim \int_{\Omega}\left\{\int_{t_{0}}^{1} \tau(t \zeta) d t\right\} d \lambda(\zeta) \\
& \lesssim \int_{\Omega}(1-t(\xi)) \tau(\xi) d \lambda(\xi)
\end{aligned}
$$


Comme $1-t(\xi)$ est majoré à une constante absolue près par $-\rho(\xi)$ et que $\tau d \lambda=\Theta \wedge \frac{\beta^{n-1}}{(n-1) !}$, l'inégalité (1) est établie.

Inégalité (2). Fixons $i$ dans $\{1, \ldots, N\}$ et posons $[z \cdot \Theta]=$ $\sum_{j, k=1}^{n} z_{j} \Theta_{j k}(z) d \bar{z}_{k}$.

$$
\begin{aligned}
\bar{\partial} \rho_{i} \wedge w_{0,1}(\zeta)=\int_{0}^{1}\left(\bar{\partial} \rho_{i} \wedge[z \cdot \Theta]\right)(t \zeta) d t \\
\quad+\int_{0}^{1}\left(\bar{\partial} \rho_{i}(\zeta)-\bar{\partial} \rho_{i}(t \zeta)\right) \wedge[z \cdot \Theta](t \zeta) d t
\end{aligned}
$$

La fonction $\rho_{i}$ étant $\mathcal{C}^{2}$ sur un voisinage de $\bar{\Omega}_{i},\left|\bar{\partial} \rho_{i}(\zeta)-\bar{\partial} \rho_{i}(t \zeta)\right| \lesssim(1-t)$, de plus sur $\tilde{\Omega}_{i}\left(-1 / \rho_{i}\right)^{1 / 2} \lesssim(-1 / \rho)^{1 / 2}\left(\rho / \rho_{i}\right)^{3 / 2}$, d'où avec $(3.8)$

$$
\begin{gathered}
\int_{\tilde{\Omega}_{i}}\left(-\frac{1}{\rho_{i}}\right)^{1 / 2}\left|\bar{\partial} \rho_{i} \wedge w_{0,1}(\zeta)\right| d \lambda(\zeta) \\
\lesssim \int_{\Omega}\left(-\frac{1}{\rho(\zeta)}\right)^{1 / 2}\left\{\int_{0}^{1}(1-t) \tau(t \zeta) d t\right\} d \lambda(\zeta) \\
+\int_{\Omega}\left(-\frac{1}{\rho(\zeta)}\right)^{1 / 2}\left(\frac{\rho}{\rho_{i}}(\zeta)\right)^{3 / 2}\left\{\int_{0}^{1}\left|\left(\bar{\partial} \rho_{i} \wedge[z \cdot \Theta]\right)(t \zeta)\right| d t\right\} d \lambda(\zeta) .
\end{gathered}
$$

Pour majorer la somme de droite nous allons de nouveau faire le changement de variable $\xi=t \zeta$. Le coefficient $\left(\rho / \rho_{i}\right)^{3 / 2}$ ne pose pas de problème car en utilisant que $-\rho_{i}$ et $-\rho$ sont comparables respectivement à $1-J_{i}$ et $1-J$, on peut montrer que $\left(\rho / \rho_{i}\right)^{3 / 2}(\xi / t)$ est majorable à une constante près par $\left(\rho / \rho_{i}\right)^{3 / 2}(\xi)$. Puis nous appliquons le Théorème de Fubini et les inégalités (3.5) et (3.6),

$$
\begin{aligned}
\int_{\tilde{\Omega}_{i}}( & \left.-\frac{1}{\rho_{i}}\right)^{1 / 2}\left|\bar{\partial} \rho_{i} \wedge w_{0,1}(\zeta)\right| d \lambda(\zeta) \lesssim \int_{\Omega}(-\rho(\xi)) \tau(\xi) d \lambda(\xi) \\
& +\int_{\Omega}(-\rho(\xi))^{1 / 2}\left(\frac{\rho}{\rho_{i}}(\xi)\right)^{3 / 2}\left|\left(\bar{\partial} \rho_{i} \wedge[z \cdot \Theta]\right)(\xi)\right| d \lambda(\xi)
\end{aligned}
$$

Enfin, avec l'inégalité de Cauchy-Schwarz

$$
(-\rho)^{1 / 2}\left(\frac{\rho}{\rho_{i}}\right)^{3 / 2}\left|\bar{\partial} \rho_{i} \wedge[z \cdot \Theta]\right| \leq-\rho \tau+\left(\frac{\rho}{\rho_{i}}\right)^{3}\left|\partial \rho_{i} \wedge \bar{\partial} \rho_{i} \wedge \Theta \wedge \beta^{n-2}\right|
$$

et (3.10), l'inégalité (2) est établie. 
Proposition 3.4. Soit $\Theta$ une $(1,1)$-forme positive et fermée, à coefficients dans $\mathcal{C}^{2}(\bar{\Omega})$. Si $\Theta$ vérifie la condition du Théorème 3.1, alors il existe une fonction $W$ solution sur $\Omega$ de $i \partial \bar{\partial} W=\Theta$ et telle que

$$
\sup _{\varepsilon>0} \int_{\partial \Omega_{\varepsilon}} W^{+} i \bar{\partial} \rho \wedge \beta^{n-1} \lesssim \sum_{i=0}^{N} B_{i}(\Theta) .
$$

Preuve: Soient $w_{0,1}$ la $(0,1)$-forme définie par la formule d'homotopie (3.3) et $U$ la solution de $\bar{\partial} U=w_{0,1}$ définie dans la Proposition-Définition 2.1, $W=U+\bar{U}$ est solution de $i \partial \bar{\partial} W=\Theta$ et, grâce au Théorème 2.7 et à la Proposition 3.3 , nous avons

$$
\forall j \in\{1, \ldots, N\}, \quad \int_{S_{j}}|W| d \sigma_{j} \lesssim \sum_{i=0}^{N} B_{i}(\Theta) .
$$

Nous allons montrer que $\forall \varepsilon>0, \int_{\partial \Omega_{\varepsilon}} W^{+} i \bar{\partial} \rho \wedge \beta^{n-1} \lesssim \sum_{j=1}^{N} \int_{S_{j}}|W| d \sigma_{j}$.

$1^{e}$ étape. Posons pour $\eta>0$,

$$
\begin{aligned}
& D_{\eta}=\bigcap_{1 \leq j<k \leq N}\left\{z \in \mathbb{C}^{n} \mid \rho_{j}(z)+\rho_{k}(z)<-\eta\right\} \text {, } \\
& \text { (3.12) } \forall \varepsilon>0 \int_{\partial \Omega_{\varepsilon}} W^{+} i \bar{\partial} \rho \wedge \beta^{n-1} \\
& \leq \lim _{\eta \downarrow 0} \int_{\Omega \cap D_{\eta}}\left(W^{+} i \partial \bar{\partial} \rho-\rho i \partial \bar{\partial} W^{+}\right) \wedge \beta^{n-1} .
\end{aligned}
$$

Ceci résulte du fait que $\left(\Omega \cap D_{\eta}\right)_{\eta>0}$ est une famille d'ouverts qui croit vers $\Omega$ lorsque $\eta \downarrow 0$ et qu'après avoir appliqué deux fois la formule de Stokes,

$$
\begin{aligned}
& \int_{\partial \Omega_{\varepsilon}} W^{+} i \bar{\partial} \rho \wedge \beta^{n-1} \leq \int_{\Omega}\left(W^{+} i \partial \bar{\partial} \rho-\rho i \partial \bar{\partial} W^{+}\right) \wedge \beta^{n-1} . \\
& 2^{e} \text { étape. } \\
& \text { 3.13) } \lim _{\eta \downarrow 0} \int_{\Omega \cap D_{\eta}}\left(W^{+} i \partial \bar{\partial} \rho-\rho i \partial \bar{\partial} W^{+}\right) \wedge \beta^{n-1} \lesssim \sum_{j=1}^{N} \int_{S_{j}} W^{+} d \sigma_{j} .
\end{aligned}
$$

Comme $\Omega \cap \partial D_{\eta}$ tend vers l'ensemble vide lorsque $\eta$ décroit vers 0 , après avoir régularisé $W^{+}$et passé à la limite, nous avons

$$
\begin{aligned}
\lim _{\eta \downarrow 0} \int_{\Omega \cap D_{\eta}}\left(W^{+} i \partial \bar{\partial} \rho-\rho i \partial \bar{\partial} W^{+}\right) \wedge \beta^{n-1} & \\
& =\lim _{\eta \downarrow 0} \int_{\partial \Omega \cap D_{\eta}} W^{+} i \bar{\partial} \rho \wedge \beta^{n-1} .
\end{aligned}
$$


$\partial \Omega \cap D_{\eta}=\bigcup_{j=1}^{N}\left(S_{j} \cap D_{\eta}\right)$ et $\operatorname{sur} S_{j} \cap D_{\eta} i \bar{\partial} \rho \equiv i \bar{\partial} \rho_{j}$, d'où

$$
\lim _{\eta \downarrow 0} \int_{\partial \Omega \cap D_{\eta}} W^{+} i \bar{\partial} \rho \wedge \beta^{n-1} \leq \sum_{j=1}^{N} \int_{S_{j}} W^{+} d \sigma_{j}
$$

Ceci termine la $2^{e}$ étape .

La proposition découle des inégalités (3.11) à (3.13).

\subsection{Régularisation.}

Soit $\varphi$ une fonction $\mathcal{C}^{\infty}$, à support compact dans $B(0,1 / 2)$, ne dépendant que de $|z|$, à valeurs dans $[0,1]$ et telle que $\int_{\mathbb{R}^{2 n}} \varphi d \lambda=1$.

Pour $\varepsilon$ fixé dans ]0,1[, nous définissons $\operatorname{sur} \mathbb{R}^{2 n}, \varphi_{\varepsilon} \operatorname{par} \varphi_{\varepsilon}(\zeta)=$ $1 / \varepsilon^{2 n} \varphi(\zeta / \varepsilon)$.

$\Omega$ étant supposé étoilé par rapport à 0 , nous pouvons définir pour tout $t$ de $] 1 / 2,1[$

$$
\forall \zeta \in \Omega_{t}=\frac{1}{t} \Omega, \quad \Theta(t \zeta)=\sum_{j, k=1}^{n} \Theta_{j k}(t \zeta) d \zeta_{j} \wedge d \bar{\zeta}_{k}
$$

Nous notons $\varepsilon_{t}=1 / 2 \operatorname{dist}\left(\partial \Omega, \partial \Omega_{t}\right)$ et nous définissons alors comme régularisée de $\Theta$

$$
\left.\Theta_{t}=\Theta(t \cdot) * \varphi_{\varepsilon_{t}}, \quad \forall t \in\right] 1 / 2,1[
$$

La convolution s'applique à chaque coefficient de $\Theta$. Nous obtenons ainsi une $(1,1)$-forme positive et fermée sur $\Omega$, à coefficients $\mathcal{C}^{\infty}$ sur un voisinage de $\bar{\Omega}$.

Proposition 3.5. Pour tout $t$ de $] 1 / 2,1[$, nous avons

$$
\begin{aligned}
B_{0}\left(\Theta_{t}\right) & \lesssim B_{0}(\Theta), \\
B_{i}\left(\Theta_{t}\right) & \lesssim B_{0}(\Theta)+B_{i}(\Theta), \quad \forall i \in\{1, \ldots, N\} .
\end{aligned}
$$

Preuve: Commençons par remarquer que pour tout $z$ de $\Omega_{t}$ tel que $B\left(z, \varepsilon_{t}\right) \cap \Omega \neq \emptyset$, dist $(t z, \partial \Omega)>\varepsilon_{t} / 2$. Ceci permet de majorer $-\rho(\zeta)$ par $-\rho(t z)$ et $\left(\rho / \rho_{i}\right)(\zeta)$ par $\left(\rho / \rho_{i}\right)(t z)$ pour tout $\zeta$ de $B\left(z, \varepsilon_{t}\right) \cap \Omega$.

Il en résulte l'inégalité (1) puisqu'avec le Théorème de Fubini

$$
\int_{\Omega}-\rho \tau_{t} d \lambda=\int_{\Omega_{t}} \tau(t z)\left(\int_{\Omega \cap B\left(z, \frac{\varepsilon_{t}}{2}\right)}-\rho(\zeta) \varphi\left(\frac{\zeta-z}{\varepsilon_{t}}\right) \frac{d \lambda(\zeta)}{\varepsilon_{t}^{2 n}}\right) d \lambda(z) .
$$


En outre

$$
\begin{aligned}
& B_{i}\left(\Theta_{t}\right) \leq \int_{\Omega}\left(\frac{\rho}{\rho_{i}}(\zeta)\right)^{3}\left(\left(\partial \rho_{i} \wedge \bar{\partial} \rho_{i}\right)(t \cdot) \wedge \Theta(t \cdot) \wedge \beta^{n-2}\right) * \varphi_{\varepsilon_{t}}(\zeta) \\
+ & \int_{\Omega}\left(\frac{\rho}{\rho_{i}}(\zeta)\right)^{3}\left\{\left(\left(\partial \rho_{i} \wedge \bar{\partial} \rho_{i}\right)(\cdot)-\left(\partial \rho_{i} \wedge \bar{\partial} \rho_{i}\right)(t \cdot)\right) \wedge \Theta(t \cdot) \wedge \beta^{n-2}\right\} * \varphi_{\varepsilon_{t}}(\zeta) \\
+ & \int_{\Omega}\left(\frac{\rho}{\rho_{i}}(\zeta)\right)^{3}\left\{\partial \rho_{i} \wedge \bar{\partial} \rho_{i} \wedge \Theta_{t} \wedge \beta^{n-2}-\left(\partial \rho_{i} \wedge \bar{\partial} \rho_{i} \wedge \Theta(t \cdot) \wedge \beta^{n-2}\right) * \varphi_{\varepsilon_{t}}\right\}(\zeta) .
\end{aligned}
$$

Il en découle l'inégalité (2) en remarquant que pour tout $z$ de $\Omega_{t},|z-t z| \lesssim$ $\varepsilon_{t}$ et dès que $B\left(z, \varepsilon_{t} / 2\right) \cap \Omega \neq \emptyset, \varepsilon_{t} \lesssim-\rho(t z)$.

Nous allons maintenant terminer la preuve du Théorème 3.1. Grâce aux Propositions 3.4 et 3.5 et sous les hypothèses du Théorème 3.1 , nous pouvons définir pour tout $t \in] 1 / 2,1\left[\right.$, une fonction $W_{t}$, plurisousharmonique sur $\Omega$, telle que

$$
i \partial \bar{\partial} W_{t}=\Theta_{t} \quad \text { et } \quad\left\|W_{t}^{+}\right\|_{N_{1}(\Omega)} \lesssim \sum_{i=0}^{N} B_{i}(\Theta) .
$$

Pour $\eta>0$ fixé, nous choisissons $\left.t_{\eta} \in\right] 1 / 2,1\left[\right.$ de sorte que $\frac{1}{t_{\eta}} \Omega_{\eta} \subset \Omega_{\eta / 2}$ et posons pour tout $t$ de $] t_{\eta}, 1\left[\right.$, tout $z$ de $\Omega_{\eta}, V_{t}(z)=t^{2} W_{t}(z / t)$. Alors $i \partial \bar{\partial} V_{t}=\Theta * \varphi_{t \varepsilon_{t}}$ sur $\Omega_{\eta}$ et

$$
\int_{\partial \Omega_{\eta^{\prime}}} V_{t}^{+} i \bar{\partial} \rho \wedge \beta^{n-1} \lesssim \sum_{i=0}^{N} B_{i}(\Theta), \quad \forall \eta^{\prime}>\eta
$$

En appliquant la formule de Stokes nous en déduisons que la famille $\left(V_{t}^{+}\right)_{t \in] t_{\eta}, 1[}$ est uniformément bornée en norme $L^{1}$ sur $\Omega_{\eta^{\prime}}$ et donc aussi $\left(V_{t}^{+}\right)_{t \in] t_{\eta}, 1[}$. Nous pouvons en extraire une suite qui converge faiblement vers $V_{\eta^{\prime}}$ et $i \partial \bar{\partial} V_{\eta^{\prime}}=\Theta$ sur $\Omega_{\eta^{\prime}}$.

Soit $G_{\eta / 2}$ la fonction de Green sur $\Omega_{\eta / 2}$, posons

$$
\forall z \in \Omega_{\eta / 2}, \quad \tilde{V}(z)=\int_{\Omega_{\eta / 2}} 2 G_{\eta / 2}(z, \zeta) d \tau(\zeta) .
$$

Quitte à supposer $\eta$ assez proche de 0 , nous pouvons définir pour tout $t$ de $] t_{\eta}, 1\left[\tilde{V}_{t}=\tilde{V} * \varphi_{t \varepsilon_{t}}\right.$ qui sera $\mathcal{C}^{\infty}$ sur $\bar{\Omega}_{\eta}$. Alors sur $\Omega_{\eta}, \triangle \tilde{V}_{t}=$ $(2 \tau) * \varphi_{t \varepsilon_{t}}=\triangle V_{t}$, d'où $V_{t}=\tilde{V}_{t}+H_{t}$ avec $H_{t}$ fonction harmonique sur $\Omega_{\eta}$. La famille $\left(H_{t}\right)_{t}$, à partir d'un certain rang, est uniformément 
bornée sur tout compact de $\Omega_{\eta}$ puisque $\tilde{V}_{t}(z) \leq \tilde{V}_{t_{0}}(z)$ pour $t \geq t_{0}$ et la famille $\left(V_{t}\right)_{t \in] t_{\eta / 2}, 1[}$ est uniformément bornée en norme $L^{1}$ sur $\Omega_{\eta}$.

$\left(H_{t}\right)_{t}$ admet une sous-suite qui converge uniformément sur tout compact de $\Omega_{\eta}$ et en prenant une sous-suite extraite commune avec $\left(V_{t}\right)_{t \in] t_{\eta / 2}, 1[}$ nous avons

$$
V_{\eta}^{+} \leq H_{\eta}^{+}+\tilde{V}_{t_{0}}^{+} \operatorname{sur} \Omega_{\eta}
$$

Par convergence dominée nous pouvons passer à la limite dans (3.17) et

$$
\forall \eta^{\prime}>\eta, \quad \int_{\partial \Omega_{\eta^{\prime}}} V_{\eta}^{+} i \bar{\partial} \rho \wedge \beta^{n-1} \lesssim \sum_{i=0}^{N} B_{i}(\Theta), \quad i \partial \bar{\partial} V_{\eta}=\Theta \text { sur } \Omega_{\eta} .
$$

Nous terminons avec un procédé classique de diagonalisation.

\section{Cas de l'intersection de deux domaines}

Dans ce paragraphe nous allons donner une condition suffisante d'appartenance à $N_{2}(\Omega)$ lorsque $\Omega$ est l'intersection transverse de deux domaines. Etre dans cette classe de Nevanlinna nous impose comme condition limite l'intégrabilité sur $S_{12}$ de notre fonction selon la mesure $i \bar{\partial} \rho_{1} \wedge i \bar{\partial} \rho_{2} \wedge \beta^{n-2}$. L'estimation est alors facilitée par le fait que $\left|i \bar{\partial} \rho_{1} \wedge i \bar{\partial} \rho_{2}\right|$ correspond exactement au Jacobien du changement de variables dont nous avons besoin. Malheureusement cela n'est plus ausi simple lorsque l'on a plus de deux domaines et nous ne savons pas le généraliser. $\bar{\Omega}$.

Dans ce paragraphe $\rho_{1}$ et $\rho_{2}$ sont supposés être $\mathcal{C}^{3}$ sur un voisinage de

\subsection{Résolution de $\bar{\partial} u=w$ et estimations.}

Comme solution du $\bar{\partial}$ nous reprenons celle définie dans la PropositionDéfinition 2.1 et précisons sa restriction à $S_{12}$. Nous notons

$$
\begin{aligned}
s(\zeta, z)=\sum_{j=1}^{n}\left(-\left(\rho_{1}+\rho_{2}\right)(z)\right. & \left(\bar{\zeta}_{j}-\bar{z}_{j}\right) \\
& \left.-\left(\bar{F}^{(1)}+\bar{F}^{(2)}\right)\left(F_{j}^{(1)}+F_{j}^{(2)}\right)(z, \zeta)\right) d \zeta_{j},
\end{aligned}
$$

$$
\begin{aligned}
& K(\zeta, z)=\sum_{\substack{\alpha_{0}, \alpha_{1}, \alpha_{2} \\
\alpha_{0}+\alpha_{1}+\alpha_{2}=n-1}} \frac{(n-1) !}{\alpha_{1} ! \alpha_{2} !} G_{1}^{\left(\alpha_{1}\right)} G_{2}^{\left(\alpha_{2}\right)} \frac{s \wedge\left(\bar{\partial}_{\zeta} s\right)^{\alpha_{0}}}{\langle s, \zeta-z\rangle^{\alpha_{0}+1}} \\
& \wedge\left(\bar{\partial} Q^{(1)}\right)^{\alpha_{1}} \wedge\left(\bar{\partial} Q^{(2)}\right)^{\alpha_{2}} .
\end{aligned}
$$


Nous conservons les notations du paragraphe II mais les $F_{j}^{(i)}$ sont maintenant $\mathcal{C}^{2}$ sur $\bar{\Omega}$.

Proposition-Définition 4.1. Soit $w$ une $(0,1)$-forme $\bar{\partial}$-fermée et à coefficients dans $\mathcal{C}^{1}(\bar{\Omega})$, une solution de $\bar{\partial} u=w$ est donnée par

(a) $\forall z \in \bar{\Omega}, k=1,2 \quad u(z)=c_{n} \int_{\Omega} w(\zeta) \wedge K_{k}(\zeta, z)=c_{n} \int_{\Omega} w(\zeta) \wedge$ $K(\zeta, z)$.

(b) Pour tout $z$ de $S_{12}, u(z)=c_{n} \int_{\Omega} w(\zeta) \wedge \tilde{K}(\zeta, z)$ avec

$$
\begin{gathered}
\tilde{K}(\zeta, z)=\sum_{k=0}^{n-1} c_{k}\left(\frac{-\rho_{1}(\zeta)}{-\rho_{1}(\zeta)+F^{(1)}(\zeta, z)}\right)^{\alpha+k}\left(\frac{-\rho_{2}(\zeta)}{-\rho_{2}(\zeta)+F^{(2)}(\zeta, z)}\right)^{\alpha+n-1-k} \\
\times \frac{\sum_{l=1}^{n}\left(F_{l}^{(1)}+F_{l}^{(2)}\right)(z, \zeta) d \zeta_{l}}{-\left(F^{(1)}+F^{(2)}\right)(z, \zeta)} \wedge\left(\bar{\partial}_{\zeta} Q^{(1)}(\zeta, z)\right)^{k} \\
\wedge\left(\bar{\partial}_{\zeta} Q^{(2)}(\zeta, z)\right)^{n-1-k}
\end{gathered}
$$

Preuve:

(a) résulte du (a) de la Proposition-Définition 2.1 et de

$$
|s(\zeta, z)| \leq C|\zeta-z|, \quad|\langle s(\zeta, z), \zeta-z\rangle| \geq c_{L}|\zeta-z|^{2}
$$

uniformément pour $\zeta \in \bar{\Omega}$ et $z \in L$ compact de $\Omega$.

(b) Pour tout $z$ de $S_{12},\left(s \wedge \bar{\partial}_{\zeta} s\right)(z, \zeta) \equiv 0$ et $\int_{\Omega} w(\zeta) \wedge K(\zeta, z)$ tend vers $\int_{\Omega} w(\zeta) \wedge \tilde{K}\left(\zeta, z_{0}\right)$ lorsque $z$ tend vers $z_{0} \in S_{12}$.

Pour estimer $\int_{S_{12}}\left|\int_{\Omega} w(\zeta) \wedge \tilde{K}(\zeta, z)\right| i \bar{\partial} \rho_{1} \wedge i \bar{\partial} \rho_{2} \wedge \beta^{n-2}(z)$ nous commençons par le majorer par $\sum_{i=1,2} \int_{S_{12}}\left|\int_{\tilde{\Omega}_{i}} w(\zeta) \wedge \tilde{K}(\zeta, z)\right| i \bar{\partial} \rho_{1} \wedge i \bar{\partial} \rho_{2} \wedge$ $\beta^{n-2}(z)$. Les deux termes se comportant de façon identique, nous ne nous occuperons que de celui portant sur $\tilde{\Omega}_{1}$.

Certaines intégrales vont être ré-exprimées à l'aide de la formule de Stokes, pour cela nous notons

$$
A_{i}(\zeta, z)=\sum_{j, l=1}^{n} \frac{\partial F_{j}^{(i)}}{\partial \bar{\zeta}_{l}}(\zeta, z) d \zeta_{j} \wedge d \bar{\zeta}_{l}, \quad i=1,2,
$$

et pour $k \in\{1, \ldots, n-1\}$

$$
\begin{aligned}
& B_{0}^{k}(\zeta, z)=-\alpha \frac{\left(-\rho_{1}(\zeta)\right)^{\alpha-1}}{\left(-\rho_{1}(\zeta)+F^{(1)}(\zeta, z)\right)^{\alpha+k}} \frac{\left(-\rho_{2}(\zeta)\right)^{\alpha}}{\left(-\rho_{2}(\zeta)+F^{(2)}(\zeta, z)\right)^{\alpha+n-1-k}} \partial \rho_{1}(\zeta) \\
& \wedge \frac{\partial \rho_{2}(\zeta)}{-\left(F^{(1)}+F^{(2)}\right)(z, \zeta)} \wedge A_{1}^{k-1}(\zeta, z) \wedge A_{2}^{n-1-k}(\zeta, z) \wedge \bar{\partial} \rho_{1}(\zeta) \wedge(-\bar{w}+w)(\zeta)
\end{aligned}
$$


(4.5)

$$
\begin{aligned}
B_{1}^{k}(\zeta, z)= & -(\alpha+k) \frac{\left(-\rho_{1}(\zeta)\right)^{\alpha}}{\left(-\rho_{1}(\zeta)+F^{(1)}(\zeta, z)\right)^{\alpha+k+1}} \frac{\left(-\rho_{2}(\zeta)\right)^{\alpha}}{\left(-\rho_{2}(\zeta)+F^{(2)}(\zeta, z)\right)^{\alpha+n-1-k}} \\
& \times\left(-\partial \rho_{1}(\zeta)+d_{\zeta} F^{(1)}(\zeta, z)\right) \wedge \frac{\partial \rho_{2}(\zeta)}{-\left(F^{(1)}+F^{(2)}\right)(z, \zeta)} \\
& \wedge A_{1}^{k-1}(\zeta, z) \wedge A_{2}^{n-1-k}(\zeta, z) \wedge \bar{\partial} \rho_{1}(\zeta) \wedge(-\bar{w}+w)(\zeta)
\end{aligned}
$$

(4.6)

$$
\begin{aligned}
& B_{2}^{k}(\zeta, z)=-\frac{\left(-\rho_{1}(\zeta)\right)^{\alpha}}{\left(-\rho_{1}(\zeta)+F^{(1)}(\zeta, z)\right)^{\alpha+k}} \frac{\left(-\rho_{2}(\zeta)\right)^{\alpha-1}}{\left(-\rho_{2}(\zeta)+F^{(2)}(\zeta, z)\right)^{\alpha+n-1-k}} \\
& \times\left(\alpha \bar{\partial} \rho_{2}(\zeta)+(\alpha+n-1-k) \frac{-\rho_{2}(\zeta)}{-\rho_{2}(\zeta)+F^{(2)}(\zeta, z)}\left(-\bar{\partial} \rho_{2}(\zeta)+d_{\zeta} F^{(2)}(\zeta, z)\right)\right) \\
& \wedge \frac{\partial \rho_{2}(\zeta)}{-\left(F^{(1)}+F^{(2)}\right)(z, \zeta)} \wedge A_{1}^{k-1}(\zeta, z) \wedge A_{2}^{n-1-k}(\zeta, z) \wedge \bar{\partial} \rho_{1}(\zeta) \wedge(-\bar{w}+w)(\zeta)
\end{aligned}
$$

$$
\begin{array}{r}
B_{3}^{k}(\zeta, z)=-\frac{\left(-\rho_{1}(\zeta)\right)^{\alpha}}{\left(-\rho_{1}(\zeta)+F^{(1)}(\zeta, z)\right)^{\alpha+k}} \frac{\left(-\rho_{2}(\zeta)\right)^{\alpha}}{\left(-\rho_{2}(\zeta)+F^{(2)}(\zeta, z)\right)^{\alpha+n-1-k}} \\
\times \frac{1}{-\left(F^{(1)}+F^{(2)}\right)(z, \zeta)}\left(\partial \bar{\partial} \rho_{2}(\zeta)+\partial \rho_{2}(\zeta) \wedge \frac{\left(d_{\zeta} F^{(1)}+d_{\zeta} F^{(2)}\right)(z, \zeta)}{-\left(F^{(1)}+F^{(2)}\right)(z, \zeta)}\right) \\
\wedge A_{1}^{k-1}(\zeta, z) \wedge A_{2}^{n-1-k}(\zeta, z) \wedge \bar{\partial} \rho_{1}(\zeta) \wedge(-\bar{w}+w)(\zeta)
\end{array}
$$

$$
\begin{aligned}
& B_{4}^{k}(\zeta, z)=\frac{\left(-\rho_{1}(\zeta)\right)^{\alpha}}{\left(-\rho_{1}(\zeta)+F^{(1)}(\zeta, z)\right)^{\alpha+k}} \frac{\left(-\rho_{2}(\zeta)\right)^{\alpha}}{\left(-\rho_{2}(\zeta)+F^{(2)}(\zeta, z)\right)^{\alpha+n-1-k}} \\
& \frac{\partial \rho_{2}(\zeta)}{-\left(F^{(1)}+F^{(2)}\right)(z, \zeta)} \wedge(-\bar{w}+w)(\zeta) \wedge d_{\zeta}\left(A_{1}^{k-1}(\zeta, z) \wedge A_{2}^{n-1-k}(\zeta, z) \wedge \bar{\partial} \rho_{1}(\zeta)\right)
\end{aligned}
$$

$$
\begin{aligned}
& B_{5}^{k}(\zeta, z)=-\frac{\left(-\rho_{1}(\zeta)\right)^{\alpha}}{\left(-\rho_{1}(\zeta)+F^{(1)}(\zeta, z)\right)^{\alpha+k}} \frac{\left(-\rho_{2}(\zeta)\right)^{\alpha}}{\left(-\rho_{2}(\zeta)+F^{(2)}(\zeta, z)\right)^{\alpha+n-1-k}} \\
& \frac{\partial \rho_{2}(\zeta)}{-\left(F^{(1)}+F^{(2)}\right)(z, \zeta)} \wedge \bar{\partial} \rho_{1}(\zeta) \wedge(-\overline{\partial w}+\partial w)(\zeta) \wedge A_{1}^{k-1}(\zeta, z) \wedge A_{2}^{n-1-k}(\zeta, z) .
\end{aligned}
$$




\section{Lemme 4.2.}

$$
\begin{aligned}
& \int_{S_{12}}\left|\int_{\tilde{\Omega}_{1}} w(\zeta) \wedge \tilde{K}(\zeta, z)\right| i \bar{\partial} \rho_{1} \wedge i \bar{\partial} \rho_{2} \wedge \beta^{n-2}(z) \lesssim \int_{\tilde{\Omega}_{1}} d \lambda(\zeta) \\
& \left\{\sum_{k=0}^{n-1} \int_{S_{12}} \frac{\left(-\rho_{1}(\zeta)\right)^{\alpha}\left(-\rho_{2}(\zeta)\right)^{\alpha}|w(\zeta)|}{\left|-\rho_{1}(\zeta)+F^{(1)}(\zeta, z)\right|^{\alpha+k}\left|-\rho_{2}(\zeta)+F^{(2)}(\zeta, z)\right|^{\alpha+n-1-k}}\right. \\
& \times \frac{i \bar{\partial} \rho_{1} \wedge i \bar{\partial} \rho_{2} \wedge \beta^{n-2}(z)}{\left|F^{(1)}(z, \zeta)+F^{(2)}(z, \zeta)\right|} \\
& +\sum_{k=0}^{n-2} \int_{S_{12}} \frac{\left(-\rho_{1}(\zeta)\right)^{\alpha}\left(-\rho_{2}(\zeta)\right)^{\alpha-1}\left|\bar{\partial} \rho_{2} \wedge w(\zeta)\right|}{\left|-\rho_{1}(\zeta)+F^{(1)}(\zeta, z)\right|^{\alpha+k}\left|-\rho_{2}(\zeta)+F^{(2)}(\zeta, z)\right|^{\alpha+n-1-k}} \\
& \times \frac{\left(\left|\partial \rho_{1} \wedge \partial \rho_{2}(\zeta)\right|+|\zeta-z|\right) i \bar{\partial} \rho_{1} \wedge i \bar{\partial} \rho_{2} \wedge \beta^{n-2}(z)}{\left|F^{(1)}(z, \zeta)+F^{(2)}(z, \zeta)\right|} \\
& +\sum_{k=1}^{n-1} \int_{S_{12}} \frac{\left(-\rho_{1}(\zeta)\right)^{\alpha-1}\left(-\rho_{2}(\zeta)\right)^{\alpha}\left|\bar{\partial} \rho_{1} \wedge w(\zeta)\right|}{\left|-\rho_{1}(\zeta)+F^{(1)}(\zeta, z)\right|^{\alpha+k}\left|-\rho_{2}(\zeta)+F^{(2)}(\zeta, z)\right|^{\alpha+n-1-k}} \\
& \times \frac{|\zeta-z| i \bar{\partial} \rho_{1} \wedge i \bar{\partial} \rho_{2} \wedge \beta^{n-2}(z)}{\left|F^{(1)}(z, \zeta)+F^{(2)}(z, \zeta)\right|} \\
& +\sum_{k=1}^{n-1} \int_{S_{12}} \sum_{i=1}^{5}\left|B_{i}^{k}(\zeta, z)\right| i \bar{\partial} \rho_{1} \wedge i \bar{\partial} \rho_{2} \wedge \beta^{n-2}(z) \\
& +\sum_{k=1}^{n-2} \int_{S_{12}} \frac{\left(-\rho_{1}(\zeta)\right)^{\alpha-1}\left(-\rho_{2}(\zeta)\right)^{\alpha-1}\left|\bar{\partial} \rho_{1} \wedge \bar{\partial} \rho_{2} \wedge w(\zeta)\right|}{\left|-\rho_{1}(\zeta)+F^{(1)}(\zeta, z)\right|^{\alpha+k}\left|-\rho_{2}(\zeta)+F^{(2)}(\zeta, z)\right|^{\alpha+n-1-k}} \\
& \left.\times \frac{|\zeta-z|\left(\left|\partial \rho_{1} \wedge \partial \rho_{2}(\zeta)\right|+|\zeta-z|\right) i \bar{\partial} \rho_{1} \wedge i \bar{\partial} \rho_{2} \wedge \beta^{n-2}(z)}{\left|F^{(1)}(z, \zeta)+F^{(2)}(z, \zeta)\right|}\right\} \\
& +\int_{\tilde{\Omega}_{2}} d \lambda(\zeta) \sum_{k=1}^{n-1} \int_{S_{12}} \sum_{i=0}^{5}\left|B_{i}^{k}(\zeta, z)\right| i \bar{\partial} \rho_{1} \wedge i \bar{\partial} \rho_{2} \wedge \beta^{n-2}(z) .
\end{aligned}
$$

L'avant dernier terme de la somme est nul pour $n=2$. 
Preuve:

$1^{e}$ étape.

$$
\begin{aligned}
& d_{\zeta}\left\{\frac{\left(-\rho_{1}(\zeta)\right)^{\alpha}}{\left(-\rho_{1}(\zeta)+F^{(1)}(\zeta, z)\right)^{\alpha+k}} \frac{\left(-\rho_{2}(\zeta)\right)^{\alpha}}{\left(-\rho_{2}(\zeta)+F^{(2)}(\zeta, z)\right)^{\alpha+n-1-k}}\right. \\
& \frac{\partial \rho_{2}(\zeta)}{-\left(F^{(1)}+F^{(2)}\right)(z, \zeta)} \wedge A_{1}^{k-1}(\zeta, z) \wedge A_{2}^{n-1-k}(\zeta, z) \\
&\left.\wedge \bar{\partial} \rho_{1}(\zeta) \wedge(-\bar{w}+w)(\zeta)\right\}=\sum_{l=0}^{5} B_{l}^{k}(\zeta, z) .
\end{aligned}
$$

Ainsi en appliquant la formule de Stokes sur $\tilde{\Omega}_{1}$ et $\tilde{\Omega}_{2}$ à la forme différentielle entre acollades, nous obtenons que son intégrale sur $\{\zeta \mid$ $\left.\rho_{1}(\zeta)=\rho_{2}(\zeta)\right\}$ vaut

$$
\int_{\tilde{\Omega}_{1}} \sum_{l=0}^{5} B_{l}^{k}(\zeta, z)=-\int_{\tilde{\Omega}_{2}} \sum_{l=0}^{5} B_{l}^{k}(\zeta, z) .
$$

$2^{e}$ étape. Pour $i=1,2$ et $p=k$ ou $n-1-k$

$$
\begin{aligned}
& \left(\bar{\partial}_{\zeta} Q^{(i)}\right)^{p}(\zeta, z)=\left(-\frac{1}{\rho_{i}(\zeta)}\right)^{p} A_{i}^{p}(\zeta, z) \\
& \quad+p\left(-\frac{1}{\rho_{i}(\zeta)}\right)^{p+1} A_{i}^{p-1}(\zeta, z) \wedge\left(\sum_{j=1}^{n} F_{j}^{(i)}(\zeta, z) d \zeta_{j}\right) \wedge \bar{\partial} \rho_{i}(\zeta)
\end{aligned}
$$

Nous écrivons de façon développée le noyau $\tilde{K}(\zeta, z)$ donné dans la Proposition-Définition 4.1 et pour tout $k$ dans $\{1, \ldots, n-1\}$ nous remplaçons $\int_{\tilde{\Omega}_{1}} B_{0}^{k}(\zeta, z)$ par $-\int_{\tilde{\Omega}_{1}} \sum_{i=1}^{5} B_{i}^{k}(\zeta, z)-\int_{\tilde{\Omega}_{2}} \sum_{i=0}^{5} B_{i}^{k}(\zeta, z)$. Puis nous majorons par une constante $\left|A_{i}(\zeta, z)\right|$ pour $i=1,2$ et nous approchons, modulo une 1-forme à coefficients d'ordre $O(|\zeta-z|)$, $\sum_{j=1}^{n} F_{j}^{(i)}(\zeta, z) d \zeta_{j}$ et $\sum_{j=1}^{n} F_{j}^{(i)}(z, \zeta) d \zeta_{j}$ par $\partial \rho_{i}(\zeta)$. Enfin nous terminons en utilisant le Théorème de Fubini.

Théorème 4.3. Pour toute $(0,1)$-forme $w$, $\bar{\partial}$-fermée sur $\Omega$, à coefficients dans $\mathcal{C}^{1}(\bar{\Omega})$, la solution de $\bar{\partial} u=w$ définie par la Proposition- 
Définition 4.1 vérifie

a) si $n=2$

$$
\begin{gathered}
\int_{S_{12}}|u(z)| i \bar{\partial} \rho_{1} \wedge i \bar{\partial} \rho_{2}(z) \lesssim \int_{\Omega}|w(\zeta)| d \lambda(\zeta) \\
+\sum_{i=1,2}\left\{\int_{\Omega} \frac{1}{\sqrt{-\rho}}\left|\bar{\partial} \rho_{i} \wedge w\right| d \lambda(\zeta)+\int_{\Omega}-\frac{1}{\rho_{1}+\rho_{2}}\left|\partial \rho_{1} \wedge \partial \rho_{2}\right|\left|\bar{\partial} \rho_{i} \wedge w\right| d \lambda(\zeta)\right\} \\
\quad+\int_{\Omega}\left|\left(\partial \rho_{2} \wedge \bar{\partial} \rho_{1}+\partial \rho_{1} \wedge \bar{\partial} \rho_{2}\right)(-\overline{\partial w}+\partial w)\right|(\zeta) d \lambda(\zeta) .
\end{gathered}
$$

b) si $n \geq 3$

$$
\begin{gathered}
\int_{S_{12}}|u(z)| i \bar{\partial} \rho_{1} \wedge i \bar{\partial} \rho_{2}(z) \wedge \beta^{n-2} \lesssim \int_{\Omega}|w(\zeta)| d \lambda(\zeta) \\
+\sum_{i=1,2}\left\{\int_{\Omega} \frac{1}{\sqrt{-\rho}}\left|\bar{\partial} \rho_{i} \wedge w\right| d \lambda(\zeta)+\int_{\Omega}-\frac{1}{\rho_{1}+\rho_{2}}\left|\partial \rho_{1} \wedge \partial \rho_{2}\right|\left|\bar{\partial} \rho_{i} \wedge w\right| d \lambda(\zeta)\right\} \\
\quad+\int_{\Omega}-\frac{1}{\rho_{1}+\rho_{2}}\left|\bar{\partial} \rho_{1} \wedge \bar{\partial} \rho_{2} \wedge w\right| d \lambda(\zeta) \\
+\int_{\Omega} \frac{\sqrt{-\rho}}{\left(\rho_{1}+\rho_{2}\right)^{2}}\left|\partial \rho_{1} \wedge \partial \rho_{2}\right|\left|\bar{\partial} \rho_{1} \wedge \bar{\partial} \rho_{2} \wedge w\right| d \lambda(\zeta) \\
+\int_{\Omega}\left|\left(\partial \rho_{2} \wedge \bar{\partial} \rho_{1}+\partial \rho_{1} \wedge \bar{\partial} \rho_{2}\right)(-\overline{\partial w}+\partial w)\right|(\zeta) d \lambda(\zeta) .
\end{gathered}
$$

Preuve: Nous majorons chaque terme obtenu dans le Lemme 4.2 en utilisant exactement les mêmes techniques qu'au paragraphe 2.4; aussi nous ne détaillerons pas les calculs et nous nous contenterons de deux remarques.

Pour tout $z$ de $S_{12}, \bar{\partial} \rho_{i}(z) \equiv-\partial \rho_{i}(z)$ pour $i=1,2$. Ainsi

$$
i \bar{\partial} \rho_{1} \wedge i \bar{\partial} \rho_{2}(z) \equiv\left(d_{z} q_{1}+\alpha_{1}\right)(\zeta, z) \wedge\left(d_{z} q_{2}+\alpha_{2}\right)(\zeta, z)
$$

où $q_{1}$ et $q_{2}$ sont les polynômes qui approchent respectivement $\Im m F^{(1)}(\zeta, z)$ et $\Im m F^{(2)}(\zeta, z)$ définis dans la Proposition-Définition 2.6, $\alpha_{1}(\zeta, z)$ et $\alpha_{2}(\zeta, z)$ sont des 1 -formes à coefficients de l'ordre de $O(|\zeta-z|)$.

Comme nous intégrons selon la mesure (4.11) nous pouvons systématiquement faire soit deux changements de variables $q_{1}=q_{1}(\zeta, z)$ et $q_{2}=$ $q_{2}(\zeta, z)$ soit, de manière équivalente quant au résultat, un changement de variables et un gain d'une puissance en $|\zeta-z|$, soit un gain de deux puissances de $|\zeta-z|$. 
Le point essentiel dans le Lemme 4.2 est que nous avons transformé l'intégrale $\int_{\tilde{\Omega}_{1}} \sum_{k=1}^{n-1} B_{0}^{k}(\zeta, z)$ en $-\sum_{i=1}^{5} \int_{\tilde{\Omega}_{1}} \sum_{k=1}^{n-1} B_{i}^{k}(\zeta, z)$ $-\sum_{i=0}^{5} \int_{\tilde{\Omega}_{2}} \sum_{k=1}^{n-1} B_{i}^{k}(\zeta, z)$, la justification vient de l'estimation de $\int_{\tilde{\Omega}_{2}} B_{0}^{k}(\zeta, z)$.

Par considération de bidegré $\left|B_{0}^{k}(\zeta, z)\right|$ est majoré à une constante absolue près par

$$
\frac{\left(-\rho_{1}(\zeta)\right)^{\alpha-1}\left(-\rho_{2}(\zeta)\right)^{\alpha}\left|\partial \rho_{1} \wedge \partial \rho_{2}(\zeta)\right|\left|\bar{\partial} \rho_{1} \wedge w(\zeta)\right|}{\left(-\rho_{1}-\rho_{2}\right)(\zeta)\left|-\rho_{1}(\zeta)+F^{(1)}(\zeta, z)\right|^{\alpha+k}\left|-\rho_{2}(\zeta)+F^{(2)}(\zeta, z)\right|^{\alpha+n-1-k}}
$$

Après changements de variables et comme içi $\zeta$ est fixé dans $\tilde{\Omega}_{2}$, nous avons

$$
\begin{aligned}
& \int_{[0, R]} \frac{\left(-\rho_{1}\right)^{\alpha-1}\left(-\rho_{2}\right)^{\alpha}}{-\rho_{1}-\rho_{2}} r^{2 n-5} d r \\
& \left\{\int_{[0, R]} \frac{d q_{1}}{\left(-\rho_{1}+q_{1}+r^{2}\right)^{\alpha+k}} \times \int_{[0, R]} \frac{d q_{2}}{\left(-\rho_{2}+q_{2}+r^{2}\right)^{\alpha+n-1-k}}\right\} \\
& \lesssim-\frac{1}{\rho_{1}+\rho_{2}}(\zeta) \int_{[0, R]} \frac{\left(-\rho_{2}\right)^{\alpha} r^{2 n-5}}{\left(-\rho_{2}+r^{2}\right)^{\alpha+n-2}} d r \lesssim-\frac{1}{\rho_{1}+\rho_{2}}(\zeta)
\end{aligned}
$$

Si nous avions traité directement $\int_{\tilde{\Omega}_{1}} \sum_{k=1}^{n-1} B_{0}^{k}(\zeta, z)$ nous l'aurions fait comme ci-dessus et aurions eu pour $k=n-1$ après intégration selon $d q_{1}$ et $d q_{2}$

$$
\int_{[0, R]} \frac{\left(-\rho_{2}\right)\left(-\rho_{1}\right)^{\alpha-1} r^{2 n-5} d r}{\left(-\rho_{1}-\rho_{2}\right)\left(-\rho_{1}+r^{2}\right)^{\alpha+n-2}} \lesssim-\frac{1}{\rho_{1}+\rho_{2}} \frac{\rho_{2}}{\rho_{1}}
$$

qui n'est pas majorable à une constante absolue près par $-\frac{1}{\rho_{1}+\rho_{2}}$ sur $\tilde{\Omega}_{1}$.

4.2 Résolution de $i d w=\Theta$.

Comme $\Omega=\Omega_{1} \cap \Omega_{2}$ est étoilé par rapport à 0 et l'intersection est $\mathbb{R}$-transverse, il existe $c_{1}<0$ et $c_{2}<0$ tels que, pour tous $c_{1}^{\prime}$ de $] c_{1}, 0[$ et $c_{2}^{\prime}$ de $] c_{2}, 0\left[\right.$, les ensembles $\left\{\rho_{1}=c_{1}^{\prime}\right\}$ et $\left\{\rho_{2}=c_{2}^{\prime}\right\}$ se coupent en une surface de dimension réelle $2 n-2$. Il existe $\eta>0$ tel que $c_{1}+2 \eta<0$ et $c_{2}+2 \eta<0$. Nous notons

$$
\begin{aligned}
D & =\left(\left\{\rho_{1}<c_{1}+\eta\right\} \cup\left\{\rho_{2}<c_{2}+\eta\right\}\right) \cap \Omega, \\
D^{\prime} & =\left\{\rho_{1}<c_{1}+2 \eta\right\} \cup\left\{\rho_{2}<c_{2}+2 \eta\right\} .
\end{aligned}
$$


La condition ci-dessus permet de dire qu'il existe un voisinage de $\left\{\rho_{1}=\right.$ $\left.\rho_{2}=0\right\}$ qui ne rencontre pas $D^{\prime}$.

Par continuité de $\rho_{1}$ et $\rho_{2}, D$ est relativement compact dans $D^{\prime}$. Nous pouvons alors définir une fonction plateau $\psi, \mathcal{C}^{\infty}$, à valeurs dans $[0,1]$, à support compact dans $D^{\prime}$ et valant identiquement 1 sur $D$.

En utilisant l'homotopie de "Cartan-Poincaré", nous pouvons définir une solution de $i d w=\Theta$ sur $D^{\prime} \cap \Omega$. Nous l'appelons $\tilde{w}$ et posons

$$
w_{2}=\psi \tilde{w}, \quad \Theta^{1}=\Theta-i d w_{2} .
$$

Ainsi, dès que nous connaitrons une solution $w_{1}$ de $i d w=\Theta^{1}, w=$ $w_{1}+w_{2}$ sera solution de $i d w=\Theta$ sur $\Omega$.

Commençons par vérifier que les estimations sur $\left(w_{2}\right)_{0,1}$ ne posent pas de problèmes. Pour $\Theta$ un $(1,1)$-courant positif à coefficients dans $\mathcal{C}^{2}(\bar{\Omega})$, nous conservons les notations de la Proposition 3.2 et posons

$$
B_{k}^{\prime}(\Theta)=\int_{\Omega} i \partial \rho_{k} \wedge \bar{\partial} \rho_{k} \wedge \Theta \wedge \beta^{n-2}, k=1,2
$$

Proposition 4.4. Soit $w_{2}$ la 1-forme définie par (4.14), alors :

a) $\int_{\Omega}\left|\left(w_{2}\right)_{0,1}\right| d \lambda \lesssim B_{0}(\Theta)$,

b) $\quad \int_{\Omega}\left(-\frac{1}{\rho}\right)^{1 / 2}\left|\bar{\partial} \rho_{k} \wedge\left(w_{2}\right)_{0,1}\right| d \lambda \lesssim B_{0}(\Theta)+B_{k}^{\prime}(\Theta), k=1,2$

c) $\quad \int_{\Omega}-\frac{1}{\rho_{1}+\rho_{2}}\left|\partial \rho_{1} \wedge \partial \rho_{2}\right|\left|\bar{\partial} \rho_{k} \wedge\left(w_{2}\right)_{0,1}\right| d \lambda \lesssim B_{0}(\Theta), \quad k=1,2$

d) $\int_{\Omega}\left|\left(\partial \rho_{2} \wedge \bar{\partial} \rho_{1}+\partial \rho_{1} \wedge \bar{\partial} \rho_{2}\right) \wedge\left(-{\overline{\partial\left(w_{2}\right)_{0,1}}}+\partial\left(w_{2}\right)_{0,1}\right)\right| d \lambda$

$$
\lesssim B_{0}(\Theta)+B_{1}^{\prime}(\Theta)+B_{2}^{\prime}(\Theta)
$$

et si $n \geq 3$

e) $\quad \int_{\Omega}-\frac{1}{\rho_{1}+\rho_{2}}\left|\bar{\partial} \rho_{1} \wedge \bar{\partial} \rho_{2} \wedge\left(w_{2}\right)_{0,1}\right| d \lambda \lesssim B_{0}(\Theta)$,

f) $\quad \int_{\Omega} \frac{\sqrt{-\rho}}{\left(\rho_{1}+\rho_{2}\right)^{2}}\left|\partial \rho_{1} \wedge \partial \rho_{2}\right|\left|\bar{\partial} \rho_{1} \wedge \bar{\partial} \rho_{2} \wedge\left(w_{2}\right)_{0,1}\right| d \lambda \lesssim B_{0}(\Theta)$.

Preuve: Puisque $D^{\prime} \cap \Omega$ est inclus dans $\Omega$ et que $\tilde{w}$ est construit à partir de l'homotopie de Poincaré, nous obtenons l'analogue de la Proposition 3.3 en remplaçant $\Omega$ par $D^{\prime} \cap \Omega$. En outre comme $-\overline{\partial \tilde{w}_{0,1}}+\partial \tilde{w}_{0,1}=$ 
$d\left(-\overline{\tilde{w}}_{0,1}+\tilde{w}_{0,1}\right)=-i \Theta$ sur $D^{\prime} \cap \Omega$, par positivité de $\Theta$

$$
\begin{aligned}
\int_{D^{\prime} \cap \Omega} \mid\left(\partial \rho_{2} \wedge \bar{\partial} \rho_{1}+\partial \rho_{1} \wedge \bar{\partial} \rho_{2}\right) \wedge\left(-\overline{\partial \tilde{w}_{0,1}}\right. & \left.+\partial \tilde{w}_{0,1}\right) \mid d \lambda \\
& \lesssim B_{1}^{\prime}(\Theta)+B_{2}^{\prime}(\Theta)
\end{aligned}
$$

Il en découle aisément les estimations pour $\left(w_{2}\right)_{0,1}$ car sur $D^{\prime} \cap \Omega$, qui contient le support de $w_{2}$, nous avons

$$
\begin{aligned}
\left|d \psi \wedge \tilde{w}_{0,1}\right| & \lesssim\left|\tilde{w}_{0,1}\right|, \\
-\frac{1}{\rho_{1}+\rho_{2}} & \leq \frac{1}{\inf \left(-c_{1},-c_{2}\right)-2 \eta} \lesssim 1 .
\end{aligned}
$$

Remarquons enfin que $\Theta^{1}=(1-\psi) \Theta-i d \psi \wedge \tilde{w}$ est encore un $(1,1)$ courant fermé à coefficients $\mathcal{C}^{2}$ sur un voisinage de $\bar{\Omega}$, mais nous avons "perdu" sur ce courant l'hypothèse de positivité. Cependant, il est la somme d'un $(1,1)$-courant positif et d'une forme à coefficients bornés en norme $L^{1}(\Omega)$ par $B_{0}(\Theta)$ et dont le support évite un voisinage de $\left\{\rho_{1}=\rho_{2}=0\right\}$. Nous verrons plus loin que ceci est suffisant pour estimer correctement $w_{1}$, et surtout, le support de $\Theta^{1}$ ne contient plus $D$ ce qui va nous permettre de définir une nouvelle homotopie.

Nous n'arrivons pas à borner les termes dont l'intégrale contient un facteur de l'ordre de $-\frac{1}{\rho_{1}+\rho_{2}}\left(\right.$ ou $\frac{\sqrt{-\rho}}{\left(\rho_{1}+\rho_{2}\right)^{2}}$ pour $n \geq 3$ ) avec l'homotopie classique. En effet, en faisant une intégration comme pour les inégalités (3.5) et (3.6), nous obtenons un terme de l'ordre de $\ln (-\rho(\zeta))$. Nous allons contourner cette difficulté en nous inspirant d'une méthode de Varopoulos [Var], présentée par Charpentier [Cha]. Celle-ci consiste à construire une famille d'homotopies, puis à faire une moyenne des solutions trouvées.

Soit $\zeta \in\left\{c_{1}<\rho_{1}<0\right\} \cap\left\{c_{2}<\rho_{2}<0\right\}$, pour tout $t$ de $\left.] 0,1\right]$ et tout $r$ de $[0,1]$, les surfaces $\left\{\rho_{1}=\rho_{1}(\zeta)\right\} \cap\left\{\rho_{2}=\rho_{2}(\zeta)\right\}$ et $\left\{\rho_{1}=\right.$ $\left.t \rho_{1}(\zeta)+(1-t) c_{1}\right\} \cap\left\{\rho_{2}=t^{1+r} \rho_{2}(\zeta)+\left(1-t^{1+r}\right) c_{2}\right\}$ sont, par hypothèse sur $c_{1}$ et $c_{2}$, de dimension $2 n-2$ et varient de façon régulière. Nous pouvons les repérer à l'aide des coordonnées $\left(\varphi_{i}\right)_{1 \leq i \leq 2 n-2}$.

Définition 4.5. Pour tout $t$ de $] 0,1]$ et tout $r$ de $[0,1]$, nous appelons $F_{t}^{r}$ le $\mathcal{C}^{1}$-difféomorphisme de $\tilde{D}=\left\{c_{1}<\rho_{1}<0\right\} \cap\left\{c_{2}<\rho_{2}<0\right\}$ dans lui-même, défini par

$$
\begin{aligned}
& \rho_{1}\left(F_{t}^{r}(\zeta)\right)=t \rho_{1}(\zeta)+(1-t) c_{1} \\
& \rho_{2}\left(F_{t}^{r}(\zeta)\right)=t^{1+r} \rho_{2}(\zeta)+\left(1-t^{1+r}\right) c_{2} \\
& \varphi_{i}\left(F_{t}^{r}(\zeta)\right)=\varphi_{i}(\zeta) i=1, \ldots, 2 n-2
\end{aligned}
$$


Le choix de $c_{1}$ et $c_{2}$ permet d'assurer que $F_{t}^{r}$ est bien bijectif sur $\left\{c_{1}<\rho_{1}<0\right\} \cap\left\{c_{2}<\rho_{2}<0\right\}$. $\rho_{1}$ et $\rho_{2}$ étant $\mathcal{C}^{2}$ sur $\bar{\Omega}, d \rho_{1}$ et $d \rho_{2}$ étant libres sur $V_{1}^{\delta} \cap V_{2}^{\delta}$, nous avons bien, avec le Théorème des fonctions implicites, $F_{t}^{r}$ et $\left(F_{t}^{r}\right)^{-1}$ de classe $\mathcal{C}^{1}$. De plus $\forall r \in[0,1], F_{1}^{r}=I d$ sur $\tilde{D}$ et il existe $\left.\left.\left.\left.t_{0} \in\right] 0,1\right], \forall t \in\right] 0, t_{0}\right] F_{t}^{r}(\tilde{D}) \subset D$ qui par construction ne rencontre pas le support de $\Theta_{1}$. Pour être parfaitement dans les conditions d'application du Lemme de Cartan-Poincaré, il faudrait que $F_{t}^{r}$ soit défini sur $\left(\left\{\rho_{1} \leq c_{1}\right\} \cup\left\{\rho_{2} \leq c_{2}\right\}\right) \cap \Omega$; or cet ensemble est inclus dans $D$. Nous pouvons définir la forme différentielle

$$
w_{r}=-i \int_{t_{0}}^{1}\left(F_{t}^{r}\right)^{*}\left(i_{Z_{t}^{r}} \Theta^{1}\right) d t
$$

où $Z_{t}^{r}$ est une famille de champs de vecteurs tels que $\frac{d}{d t} F_{t}^{r}(z)=Z_{t}^{r}\left(F_{t}^{r}(z)\right)$ $(0<t \leq 1,0 \leq r \leq 1)$ et $i_{Z_{t}^{r}} \Theta^{1}$ est défini par $i_{Z_{t}^{r}}\left(d z_{i} \wedge d \bar{z}_{j}\right)=\left(Z_{t}^{r}\right)^{i} d \bar{z}_{j}-$ $\left(Z_{t}^{r}\right)^{j} d z_{i}$

Nous appliquons le "Lemme de Cartan-Poincaré" et avons

$$
\forall r \in[0,1], \quad i d w_{r}=\Theta^{1} \text { sur } \Omega,
$$

et nous définissons

$$
w_{1}=\int_{0}^{1} w_{r} d r, \quad i d w_{1}=\Theta^{1} \operatorname{sur} \Omega
$$

Nous allons maintenant voir que $\left(w_{1}\right)_{0,1}$ vérifie de "bonnes estimations".

4.3 Estimations de la solution de $i d w_{1}=\Theta^{1}$.

Exprimons $\left(F_{t}^{r}\right)^{-1}$ à l'aide des fonctions $\rho_{1}$ et $\rho_{2}$, pour tout $\zeta$ de $\tilde{D}$

$$
\begin{aligned}
& \rho_{1}\left(\left(F_{t}^{r}\right)^{-1}(\zeta)\right)=\frac{1}{t} \rho_{1}(\zeta)+\left(1-\frac{1}{t}\right) c_{1}, \\
& \rho_{2}\left(\left(F_{t}^{r}\right)^{-1}(\zeta)\right)=\frac{1}{t^{1+r}} \rho_{1}(\zeta)+\left(1-\frac{1}{t^{1+r}}\right) c_{2} .
\end{aligned}
$$

Définition 4.6. Pour tout $r \in[0,1]$, tout $\zeta$ fixé dans $\tilde{D}$, nous posons

$$
\begin{aligned}
t_{r}(\zeta) & =\inf \left\{t \in\left[t_{0}, 1\right] \mid\left(F_{t}^{r}\right)^{-1}(\zeta) \in \tilde{D}\right\} \\
& =\max \left\{t_{0}, 1-\frac{\rho_{1}(\zeta)}{c_{1}},\left(1-\frac{\rho_{2}(\zeta)}{c_{2}}\right)^{\frac{1}{1+r}}\right\}
\end{aligned}
$$

Commençons par établir une série de Lemmes qui nous servirons pour les estimations. 
Lemme 4.7. Pour tout $\zeta$ de $\Omega \backslash D$, tout $r$ de $[0,1]$

$$
\int_{t_{r}(\zeta)}^{1}\left(\frac{1}{-\rho \circ\left(F_{t}^{r}\right)^{-1}(\zeta)}\right)^{1 / 2} d t \lesssim(-\rho(\zeta))^{1 / 2}
$$

Preuve:

$$
\text { (4.23) } \begin{aligned}
\frac{d}{d t}\left(-\rho \circ\left(F_{t}^{r}\right)^{-1}\right)^{1 / 2} & \\
& =\frac{1}{2} \frac{-1}{\left(-\rho \circ\left(F_{t}^{r}\right)^{-1}\right)^{1 / 2}}\left\langle d \rho \circ\left(F_{t}^{r}\right)^{-1}, \frac{d}{d t}\left(F_{t}^{r}\right)^{-1}\right\rangle,
\end{aligned}
$$

où $\langle$,$\rangle désigne le produit scalaire dans \mathbb{R}^{2 n}$.

En dérivant par rapport à $t$ les égalités (4.21) et (4.22), nous avons

(4.24) $\frac{d}{d t}\left(\rho_{1} \circ\left(F_{t}^{r}\right)^{-1}\right)=-\frac{1}{t^{2}}\left(\rho_{1}-c_{1}\right)=\left\langle d \rho_{1} \circ\left(F_{t}^{r}\right)^{-1}, \frac{d}{d t}\left(F_{t}^{r}\right)^{-1}\right\rangle$,

(4.25) $\frac{d}{d t}\left(\rho_{2} \circ\left(F_{t}^{r}\right)^{-1}\right)=-\frac{1+r}{t^{2+r}}\left(\rho_{2}-c_{2}\right)=\left\langle d \rho_{2} \circ\left(F_{t}^{r}\right)^{-1}, \frac{d}{d t}\left(F_{t}^{r}\right)^{-1}\right\rangle$,

ce qui, avec l'égalité (4.23), nous donne

$$
\begin{aligned}
\frac{d}{d t}\left(-\rho \circ\left(F_{t}^{r}\right)^{-1}\right)^{1 / 2} \geq & \frac{1}{2} \frac{1}{\left(-\rho \circ\left(F_{t}^{r}\right)^{-1}(\zeta)\right)^{1 / 2}} \\
\times & \left(\left(\frac{\rho_{2}}{\rho_{1}+\rho_{2}}\right)^{2} \circ\left(F_{t}^{r}\right)^{-1}(\zeta)\left(\rho_{1}(\zeta)-c_{1}\right)\right. \\
& \left.+\left(\frac{\rho_{1}}{\rho_{1}+\rho_{2}}\right)^{2} \circ\left(F_{t}^{r}\right)^{-1}(\zeta)\left(\rho_{2}(\zeta)-c_{2}\right)\right) .
\end{aligned}
$$

Soit pour tout $\zeta$ de $\Omega \backslash D, \frac{1}{\left(-\rho \circ\left(F_{t}^{r}\right)^{-1}(\zeta)\right)^{1 / 2}} \leq \frac{4}{\eta} \frac{d}{d t}\left(-\rho \circ\left(F_{t}^{r}\right)^{-1}(\zeta)\right)^{1 / 2}$.

Lemme 4.8. $\forall r \in[0,1], \forall t \in\left[t_{r}(\zeta), 1\right]$,

(i) $\quad \forall \zeta \in(\Omega \backslash D) \cap\left\{-\rho_{1} \leq \frac{c_{1}}{c_{2}}\left(-\rho_{2}\right)\right\}, \quad \frac{\rho_{1}}{\rho_{2}}\left(\left(F_{t}^{r}\right)^{-1}(\zeta)\right) \leq \frac{\rho_{1}}{\rho_{2}}(\zeta)$,

(ii) $\quad \forall \zeta \in(\Omega \backslash D) \cap\left\{-\rho_{2} \leq \frac{c_{2}}{c_{1}}\left(-\rho_{1}\right)\right\}, \quad \frac{\rho_{2}}{\rho_{1}}\left(\left(F_{t}^{r}\right)^{-1}(\zeta)\right) \leq \frac{\rho_{2}}{\rho_{1}}(\zeta)$.

Preuve: Lorsque l'on fixe $\zeta$ dans $(\Omega \backslash D) \cap\left\{-\rho_{1} \leq \frac{c_{1}}{c_{2}}\left(-\rho_{2}\right)\right\}$, la fonction f définie sur $\left[t_{r}(\zeta), 1\right]$ par $f(t)=\frac{\rho_{1}}{\rho_{2}}\left(\left(F_{t}^{r}\right)^{-1}(\zeta)\right)$ est croissante sur $\left[t_{r}(\zeta), 1\right]$. 
Corollaire 4.9. Pour tout $\zeta$ de $\Omega \backslash D$, tout $r$ de $[0,1]$, tout $t$ de $\left[t_{r}(\zeta), 1\right]$ fixés

$$
\begin{aligned}
\frac{\rho_{1}}{\rho_{2}+\rho_{1}}\left(\left(F_{t}^{r}\right)^{-1}(\zeta)\right) & \lesssim \frac{\rho_{1}}{\rho_{2}+\rho_{1}}(\zeta) \\
\frac{\rho_{2}}{\rho_{2}+\rho_{1}}\left(\left(F_{t}^{r}\right)^{-1}(\zeta)\right) & \lesssim \frac{\rho_{2}}{\rho_{2}+\rho_{1}}(\zeta) .
\end{aligned}
$$

Preuve: Il suffit de considérer le cas où $\zeta$ est fixé dans $(\Omega \backslash D) \cap\left\{-\rho_{1} \leq\right.$ $\left.\frac{c_{1}}{c_{2}}\left(-\rho_{2}\right)\right\}$, alors on applique le Lemme précédent à

$$
\frac{\frac{\rho_{1}}{\rho_{2}}\left(\left(F_{t}^{r}\right)^{-1}(\zeta)\right)}{\frac{\rho_{1}}{\rho_{2}}\left(\left(F_{t}^{r}\right)^{-1}(\zeta)\right)+1}=\frac{\rho_{1}}{\rho_{2}+\rho_{1}}\left(\left(F_{t}^{r}\right)^{-1}(\zeta)\right) .
$$

Lemme 4.10. Il existe $c_{1}^{\prime}<0, c_{2}^{\prime}<0$ tels que pour tout $r$ fixé dans $[0,1]$, nous avons

(i) $\forall \zeta \in(\Omega \backslash D) \cap\left\{-\rho_{1} \leq \frac{c_{1}^{\prime}}{c_{2}}\left(-\rho_{2}\right)\right\}$,

$$
\int_{t_{r}(\zeta)}^{1} \frac{\left(-\rho_{1}\right)^{1 / 2}}{\left(\rho_{1}+\rho_{2}\right)^{2}}\left(\left(F_{t}^{r}\right)^{-1}(\zeta)\right) d t \lesssim \frac{\left(-\rho_{1}\right)^{3 / 2}}{\left(\rho_{1}+\rho_{2}\right)^{2}}(\zeta) .
$$

(ii) $\forall \zeta \in(\Omega \backslash D) \cap\left\{-\rho_{2} \leq \frac{c_{2}^{\prime}}{c_{1}}\left(-\rho_{1}\right)\right\}$,

$$
\int_{t_{r}(\zeta)}^{1} \frac{\left(-\rho_{2}\right)^{1 / 2}}{\left(\rho_{1}+\rho_{2}\right)^{2}}\left(\left(F_{t}^{r}\right)^{-1}(\zeta)\right) d t \lesssim \frac{\left(-\rho_{2}\right)^{3 / 2}}{\left(\rho_{1}+\rho_{2}\right)^{2}}(\zeta) .
$$

Preuve: Nous choisissons $c_{1}^{\prime}<0$ assez proche de 0 , de sorte que, comme pour le Lemme 4.7, $\frac{\left(-\rho_{1}\right)^{1 / 2}}{\left(\rho_{1}+\rho_{2}\right)^{2}}\left(\left(F_{t}^{r}\right)^{-1}(\zeta)\right)$ soit majorable à une constante absolue près par $\frac{d}{d t}\left(\frac{\left(-\rho_{1}\right)^{3 / 2}}{\left(\rho_{1}+\rho_{2}\right)^{2}} \circ\left(F_{t}^{r}\right)^{-1}(\zeta)\right)$.

Voici maintenant les estimations que nous ne savions pas établir sans l'intervention de la nouvelle homotopie.

Lemme 4.11. Pour tout $\zeta$ de $\Omega \backslash D$
a) $\int_{0}^{1} \int_{t_{r}(\zeta)}^{1}-\frac{1}{\rho_{1}+\rho_{2}}\left(\left(F_{t}^{r}\right)^{-1}(\zeta)\right) d t d r \lesssim 1$
b) $\quad \int_{0}^{1} \int_{t_{r}(\zeta)}^{1} \frac{1}{\left(-\rho_{1}-\rho_{2}\right)^{3 / 2}}\left(\left(F_{t}^{r}\right)^{-1}(\zeta)\right) d t d r \lesssim \frac{1}{\left(-\rho_{1}-\rho_{2}\right)^{1 / 2}}(\zeta)$ 
Preuve: Nous nous sommes inspirés de la preuve de Lemme 15 de [Cha]. Nous posons

$$
\begin{aligned}
& I_{r}^{1}(\zeta)=\int_{t_{r}(\zeta)}^{1}-\frac{1}{\rho_{1}+\rho_{2}}\left(\left(F_{t}^{r}\right)^{-1}(\zeta)\right) d t, \quad I^{1}(\zeta)=\int_{0}^{1} I_{r}^{1}(\zeta) d r \\
& I_{r}^{2}(\zeta)=\int_{t_{r}(\zeta)}^{1} \frac{1}{\left(-\rho_{1}-\rho_{2}\right)^{3 / 2}}\left(\left(F_{t}^{r}\right)^{-1}(\zeta)\right) d t, \quad I^{2}(\zeta)=\int_{0}^{1} I_{r}^{2}(\zeta) d r
\end{aligned}
$$

Avec les égalités (4.21) et (4.22), nous avons pour tout $t$ de $\left[t_{r}(\zeta), 1\right]$

$$
\begin{aligned}
-\frac{1}{\rho_{1}+\rho_{2}} & \left(\left(F_{t}^{r}\right)^{-1}(\zeta)\right) \\
& =\frac{t^{1+r}}{-c_{1} t^{r}\left(t-\left(1-\rho_{1}(\zeta) / c_{1}\right)\right)-c_{2}\left(t^{1+r}-\left(1-\rho_{2}(\zeta) / c_{2}\right)\right)} .
\end{aligned}
$$

- Si $1-\frac{\rho_{1}(\zeta)}{c_{1}} \leq\left(1-\frac{\rho_{2}(\zeta)}{c_{2}}\right)^{\frac{1}{1+r}}$

$$
\begin{aligned}
I_{r}^{1}(\zeta) & \leq \int_{\left(1-\frac{\rho_{2}(\zeta)}{c_{2}}\right)^{\frac{1}{1+r}}}^{1}-\frac{1}{c_{1}} \frac{t}{t-\left(1-\rho_{1}(\zeta) / c_{1}\right)} d t \\
& \lesssim \ln \left(\frac{1-\left(1-\rho_{1}(\zeta) / c_{1}\right)}{\left(1-\rho_{2}(\zeta) / c_{2}\right)^{\frac{1}{1+r}}-\left(1-\rho_{1}(\zeta) / c_{1}\right)}\right)
\end{aligned}
$$

De même

$$
\begin{gathered}
I_{r}^{2}(\zeta) \leq \int_{\left(1-\frac{\rho_{2}(\zeta)}{c_{2}}\right)^{\frac{1}{1+r}}}^{1}\left(\frac{1}{-c_{1}}\right)^{3 / 2}\left(\frac{1}{t-\left(1-\rho_{1}(\zeta) / c_{1}\right)}\right)^{3 / 2} d t \\
\quad \lesssim\left(\frac{-c_{1}}{-\rho_{1}(\zeta)}\right)^{1 / 2}\left(\frac{1-\left(1-\rho_{1}(\zeta) / c_{1}\right)}{\left(1-\rho_{2}(\zeta) / c_{2}\right)^{\frac{1}{1+r}}-\left(1-\rho_{1}(\zeta) / c_{1}\right)}\right)^{1 / 2} . \\
\bullet \operatorname{Si~}\left(1-\frac{\rho_{2}(\zeta)}{c_{2}}\right)^{\frac{1}{1+r}} \leq 1-\frac{\rho_{1}(\zeta)}{c_{1}}, \\
I_{r}^{1}(\zeta) \leq \int_{1-\frac{\rho_{1}(\zeta)}{c_{1}}}^{1} \frac{1}{c_{2}} \frac{t^{1+r}}{t^{1+r}-\left(1-\rho_{2}(\zeta) / c_{2}\right)} d t \\
\lesssim \ln \left(\frac{1-\left(1-\rho_{2}(\zeta) / c_{2}\right)}{\left(1-\rho_{1}(\zeta) / c_{1}\right)^{1+r}-\left(1-\rho_{2}(\zeta) / c_{2}\right)}\right)
\end{gathered}
$$


De même

$$
\begin{aligned}
I_{r}^{2}(\zeta) & \leq \int_{1-\frac{\rho_{1}(\zeta)}{c_{1}}}^{1}\left(\frac{1}{-c_{2}}\right)^{3 / 2} \frac{t^{r}}{\left(t^{1+r}-\left(1-\rho_{2}(\zeta) / c_{2}\right)\right)^{3 / 2}} d t \\
& \lesssim\left(\frac{-c_{2}}{-\rho_{2}(\zeta)}\right)^{1 / 2}\left(\frac{1-\left(1-\rho_{2}(\zeta) / c_{2}\right)}{\left(1-\rho_{1}(\zeta) / c_{1}\right)^{1+r}-\left(1-\rho_{2}(\zeta) / c_{2}\right)}\right)^{1 / 2} .
\end{aligned}
$$

Cela nous amène à distinguer trois cas.

Cas 1. $1-\frac{\rho_{1}(\zeta)}{c_{1}} \leq 1-\frac{\rho_{2}(\zeta)}{c_{2}}$.

Alors pour tout $r$ de $[0,1] 1-\frac{\rho_{1}(\zeta)}{c_{1}} \leq\left(1-\frac{\rho_{2}(\zeta)}{c_{2}}\right)^{\frac{1}{1+r}}$ et

$$
\begin{aligned}
I^{1}(\zeta) & \lesssim \int_{0}^{1}-\frac{1}{1+r} \ln \left(1-\frac{\rho_{2}(\zeta)}{c_{2}}\right) d r \\
& +\int_{0}^{1} \ln \left(\frac{1-\left(1-\rho_{1}(\zeta) / c_{1}\right)}{1-\left(1-\rho_{1}(\zeta) / c_{1}\right)^{1-\frac{1}{1+r}}}\right) d r \\
I^{2}(\zeta) & \lesssim\left(\frac{1}{-\rho_{1}(\zeta)}\right)^{1 / 2} \frac{1}{\left(1-\rho_{1}(\zeta) / c_{1}\right)^{1 / 2}} \\
& \times \int_{0}^{1}\left(\frac{1-\left(1-\rho_{1}(\zeta) / c_{1}\right)}{1-\left(1-\rho_{1}(\zeta) / c_{1}\right)^{1-\frac{1}{1+r}}}\right)^{1 / 2} d r .
\end{aligned}
$$

Avec l'inégalité des accroissements finis appliquée à $x \rightarrow x^{\frac{r}{1+r}}$ sur $[1-$ $\left.\rho_{1}(\zeta) / c_{1}, 1\right]$, nous avons

$$
\begin{aligned}
I^{1}(\zeta) & \lesssim \ln \left(-c_{2} / \eta\right) \ln 2+\int_{0}^{1} \ln \left(\frac{1+r}{r}\right) d r \lesssim 1 \\
I^{2}(\zeta) & \lesssim\left(\frac{-c_{1}}{\eta}\right)^{1 / 2}\left(\frac{1}{-\rho_{1}(\zeta)}\right)^{1 / 2} \int_{0}^{1}\left(\frac{1+r}{r}\right)^{1 / 2} d r \lesssim\left(\frac{1}{-\rho_{1}(\zeta)}\right)^{1 / 2} \\
& \lesssim\left(1+\frac{c_{2}}{c_{1}}\right)^{1 / 2}\left(-\frac{1}{\rho_{1}+\rho_{2}}(\zeta)\right)^{1 / 2} .
\end{aligned}
$$

Cas 2. $1-\frac{\rho_{2}(\zeta)}{c_{2}} \leq\left(1-\frac{\rho_{1}(\zeta)}{c_{1}}\right)^{2}$. 
Alors pour tout $r$ de $[0,1], 1-\frac{\rho_{2}(\zeta)}{c_{2}} \leq\left(1-\frac{\rho_{1}(\zeta)}{c_{1}}\right)^{1+r}$ et

$$
\begin{aligned}
I^{1}(\zeta) & \lesssim \int_{0}^{1}-(1+r) \ln \left(1-\frac{\rho_{1}(\zeta)}{c_{1}}\right) d r \\
& +\int_{0}^{1} \ln \left(\frac{1-\left(1-\rho_{2}(\zeta) / c_{2}\right)}{1-\left(1-\rho_{2}(\zeta) / c_{2}\right)^{1-\frac{1+r}{2}}}\right) d r \\
I^{2}(\zeta) & \lesssim\left(\frac{1}{-\rho_{2}(\zeta)}\right)^{1 / 2} \frac{1}{\left(1-\rho_{2}(\zeta) / c_{2}\right)^{1 / 2}} \\
& \times \int_{0}^{1}\left(\frac{1-\left(1-\rho_{2}(\zeta) / c_{2}\right)}{1-\left(1-\rho_{2}(\zeta) / c_{2}\right)^{1-\frac{1+r}{2}}}\right)^{1 / 2} d r
\end{aligned}
$$

Avec l'inégalité des accroissements finis appliquée à $x \rightarrow x^{\frac{1-r}{2}}$ sur [1$\left.\rho_{2}(\zeta) / c_{2}, 1\right]$, nous avons

$$
\begin{aligned}
I^{1}(\zeta) & \lesssim 2 \ln \left(-c_{1} / \eta\right)+\int_{0}^{1} \ln \left(\frac{2}{1-r}\right) d r \lesssim 1 \\
I^{2}(\zeta) & \lesssim\left(\frac{-c_{2}}{\eta}\right)^{1 / 2}\left(\frac{1}{-\rho_{2}(\zeta)}\right)^{1 / 2} \int_{0}^{1}\left(\frac{2}{1-r}\right)^{1 / 2} d r \lesssim\left(\frac{1}{-\rho_{2}(\zeta)}\right)^{1 / 2} \\
& \lesssim\left(1+\frac{c_{1}}{c_{2}}\right)^{1 / 2}\left(-\frac{1}{\rho_{1}+\rho_{2}}(\zeta)\right)^{1 / 2}
\end{aligned}
$$

Cas 3. $\left(1-\frac{\rho_{1}(\zeta)}{c_{1}}\right)^{2}<1-\frac{\rho_{2}(\zeta)}{c_{2}}<1-\frac{\rho_{1}(\zeta)}{c_{1}}$.

Alors il existe $\left.r_{0} \in\right] 0,1\left[\right.$ tel que $1-\frac{\rho_{2}(\zeta)}{c_{2}}=\left(1-\frac{\rho_{1}(\zeta)}{c_{1}}\right)^{1+r_{0}}$. Nous découpons les intégrales de la variable $r$ en une portant sur $\left[0, r_{0}\right]$ et une $\operatorname{sur}\left[r_{0}, 1\right]$.

Pour $r \in\left[0, r_{0}\right]$, nous faisons les majorations comme dans le cas 2 et en particulier appliquons l'inégalité des accroissements finis à $x \rightarrow x^{\frac{r_{0}-r}{1+r_{0}}}$ $\operatorname{sur}\left[1-\rho_{2}(\zeta) / c_{2}, 1\right]$.

Pour $r \in\left[r_{0}, 1\right]$, nous faisons les majorations comme dans le cas 1 et en particulier appliquons l'inégalité des accroissements finis à $x \rightarrow x^{\frac{r-r_{0}}{1+r}}$ $\operatorname{sur}\left[1-\rho_{1}(\zeta) / c_{1}, 1\right]$.

Nous avons

$$
\begin{aligned}
I^{1}(\zeta) \lesssim\left(1+r_{0}\right) \ln \left(-c_{1} / \eta\right) & +\int_{0}^{r_{0}} \ln \left(\frac{1+r_{0}}{r_{0}-r}\right) d r \\
& +\ln 2 \ln \left(-c_{2} / \eta\right)+\int_{r_{0}}^{1} \ln \left(\frac{1+r}{r-r_{0}}\right) d r \lesssim 1
\end{aligned}
$$


et

$$
\begin{aligned}
I^{2}(\zeta) & \lesssim\left(\frac{1}{-\rho_{2}(\zeta)}\right)^{1 / 2} \int_{0}^{r_{0}}\left(\frac{1+r_{0}}{r_{0}-r}\right)^{1 / 2} d r+\left(\frac{1}{-\rho_{1}(\zeta)}\right)^{1 / 2} \int_{r_{0}}^{1}\left(\frac{1+r}{r-r_{0}}\right)^{1 / 2} d r \\
& \lesssim\left(\frac{1}{-\rho_{2}(\zeta)}\right)^{1 / 2}+\left(\frac{1}{-\rho_{1}(\zeta)}\right)^{1 / 2} \\
& \lesssim\left(\left(1+\frac{c_{1}}{c_{2}}\right)^{1 / 2}+\left(1+\frac{c_{2}}{c_{1}}\right)^{1 / 2}\right)\left(-\frac{1}{\rho_{1}+\rho_{2}}(\zeta)\right)^{1 / 2} \\
& \lesssim\left(-\frac{1}{\rho_{1}+\rho_{2}}(\zeta)\right)^{1 / 2} .
\end{aligned}
$$

Le Lemme est établi.

Lemme 4.12. Pour tout $\zeta$ de $\Omega \backslash D$ fixé
a) $\int_{0}^{1} \int_{t_{r}(\zeta)}^{1}(1-t)\left(1+\left(\frac{1}{-\rho}\right)^{1 / 2}-\frac{1}{\rho_{1}+\rho_{2}}\right)\left(\left(F_{t}^{r}\right)^{-1}(\zeta)\right) d t d r \lesssim-\rho(\zeta)$,
b) $\int_{0}^{1} \int_{t_{r}(\zeta)}^{1}(1-t)\left(-\frac{1}{\rho_{1}+\rho_{2}}\right)^{3 / 2}\left(\left(F_{t}^{r}\right)^{-1}(\zeta)\right) d t d r \lesssim \sqrt{-\rho(\zeta)}$,
c) $\int_{0}^{1} \int_{t_{r}(\zeta)}^{1}(1-t)^{2}\left(-\frac{1}{\rho_{1}+\rho_{2}}\right)^{3 / 2}\left(\left(F_{t}^{r}\right)^{-1}(\zeta)\right) d t d r \lesssim-\rho(\zeta)$.

Preuve: Pour tout $r$ de $[0,1], 1-t_{r}(\zeta) \lesssim-\rho(\zeta)$ et nous appliquons les Lemmes 4.7 et 4.11 .

\section{Posons}

$$
B_{12}(\Theta)=\int_{\Omega}-\frac{\rho_{1}^{3} \rho_{2}^{3}}{\left(\rho_{1}+\rho_{2}\right)^{7}}(\zeta) i \partial \rho_{1} \wedge \bar{\partial} \rho_{1} \wedge i \partial \rho_{2} \wedge \bar{\partial} \rho_{2} \wedge \Theta \wedge \beta^{n-3}(\zeta)
$$

Le reste du paragraphe va consister en la preuve de la 
Proposition 4.13. Soit $w_{1}$ la 1-forme définie en (4.20), alors :

(a) $\int_{\Omega}\left|\left(w_{1}\right)_{0,1}(\zeta)\right| d \lambda(\zeta) \lesssim B_{0}(\Theta)$

(b) $\int_{\Omega}\left(-\frac{1}{\rho(\zeta)}\right)^{1 / 2}\left|\bar{\partial} \rho_{k} \wedge\left(w_{1}\right)_{0,1}(\zeta)\right| d \lambda(\zeta)$

$$
\lesssim B_{0}(\Theta)+B_{k}^{\prime}(\Theta), k=1,2
$$

(c) $\int_{\Omega}-\frac{1}{\rho_{1}+\rho_{2}}(\zeta)\left|\partial \rho_{1} \wedge \partial \rho_{2}(\zeta)\right|\left|\bar{\partial} \rho_{k} \wedge\left(w_{1}\right)_{0,1}(\zeta)\right| d \lambda(\zeta)$

$$
\lesssim B_{0}(\Theta)+B_{1}^{\prime}(\Theta)+B_{2}^{\prime}(\Theta), k=1,2
$$

(d) $\int_{\Omega}\left|\left(\partial \rho_{2} \wedge \bar{\partial} \rho_{1}+\partial \rho_{1} \wedge \bar{\partial} \rho_{2}\right) \wedge\left(-{\overline{\partial\left(w_{1}\right)_{0,1}}}+\partial\left(w_{1}\right)_{0,1}\right)\right| d \lambda(\zeta)$

$$
\lesssim B_{0}(\Theta)+B_{1}^{\prime}(\Theta)+B_{2}^{\prime}(\Theta)
$$

Et si $n \geq 3$

(e) $\int_{\Omega}-\frac{1}{\rho_{1}+\rho_{2}}\left|\bar{\partial} \rho_{1} \wedge \bar{\partial} \rho_{2} \wedge\left(w_{1}\right)_{0,1}\right| d \lambda(\zeta)$

$$
\lesssim B_{0}(\Theta)+B_{1}^{\prime}(\Theta)+B_{2}^{\prime}(\Theta)
$$

(f) $\int_{\Omega} \frac{\sqrt{-\rho}}{\left(\rho_{1}+\rho_{2}\right)^{2}}\left|\partial \rho_{1} \wedge \partial \rho_{2}\right|\left|\bar{\partial} \rho_{1} \wedge \bar{\partial} \rho_{2} \wedge\left(w_{1}\right)_{0,1}\right| d \lambda(\zeta)$

$$
\lesssim B_{0}(\Theta)+B_{1}^{\prime}(\Theta)+B_{2}^{\prime}(\Theta)+B_{12}(\Theta)
$$

Avant de commencer la preuve, établissons deux Lemmes qui nous seront utiles.

Lemme 4.14. Pour tout $t$ de $] 0,1]$, tout $r$ de [0,1], la famille de champs de vecteurs $\left(Z_{t}^{r}\right)$ définie sur $\tilde{D}$ par $\frac{d}{d t} F_{t}^{r}(z)=Z_{t}^{r}\left(F_{t}^{r}(z)\right)$ est telle que

$$
Z_{t}^{r}(\cdot)=\frac{1}{t} Z^{r}(\cdot)
$$

où $\left(Z^{r}\right)_{r \in[0,1]}$ est une famille de champs de vecteurs $\mathcal{C}^{0}$ sur $\Omega \backslash D$.

Preuve: D'après la Définition 4.5 de $F_{t}^{r}$ et avec le Théorème des fonctions implicites, nous pouvons trouver localement une fonction $F$ qui est $\mathcal{C}^{1}$ de $\mathbb{R}^{2 n}$ dans $\mathbb{R}^{2 n}$ et telle que

$$
F_{t}^{r}(\zeta)=F\left(\rho_{1}\left(F_{t}^{r}(\zeta)\right), \rho_{2}\left(F_{t}^{r}(\zeta)\right), \varphi_{1}(\zeta), \ldots, \varphi_{2 n-2}(\zeta)\right)
$$


Ainsi :

$$
\begin{gathered}
\frac{d}{d t} F_{t}^{r}(\zeta)=\frac{\partial F}{\partial x_{1}}\left(\rho_{1}\left(F_{t}^{r}(\zeta)\right), \rho_{2}\left(F_{t}^{r}(\zeta)\right), \varphi_{1}(\zeta), \ldots, \varphi_{2 n-2}(\zeta)\right)\left(\rho_{1}(\zeta)-c_{1}\right) \\
+\frac{\partial F}{\partial x_{2}}\left(\rho_{1}\left(F_{t}^{r}(\zeta)\right), \rho_{2}\left(F_{t}^{r}(\zeta)\right), \varphi_{1}(\zeta), \ldots, \varphi_{2 n-2}(\zeta)\right)(1+r)\left(\rho_{2}(\zeta)-c_{2}\right) t^{r}
\end{gathered}
$$

Et comme $\rho_{1}(\zeta)-c_{1}=\frac{1}{t}\left(\rho_{1}\left(F_{t}^{r}(\zeta)\right)-c_{1}\right), t^{r}\left(\rho_{2}(\zeta)-c_{2}\right)=\frac{1}{t}\left(\rho_{2}\left(F_{t}^{r}(\zeta)\right)-\right.$ $\left.c_{2}\right)$, en posant

$$
\begin{aligned}
Z^{r}(\cdot)= & \left(\rho_{1}(\cdot)-c_{1}\right) \frac{\partial F}{\partial x_{1}}\left(\rho_{1}(\cdot), \rho_{2}(\cdot), \varphi_{1}(\cdot), \ldots, \varphi_{2 n-2}(\cdot)\right) \\
& +(1+r)\left(\rho_{2}(\cdot)-c_{2}\right) \frac{\partial F}{\partial x_{2}}\left(\rho_{1}(\cdot), \rho_{2}(\cdot), \varphi_{1}(\cdot), \ldots, \varphi_{2 n-2}(\cdot)\right)
\end{aligned}
$$

nous avons $\frac{d}{d t} F_{t}^{r}(\zeta)=\frac{1}{t} Z^{r}\left(F_{t}^{r}(\zeta)\right)$.

$F_{t}^{r}$ étant $\mathcal{C}^{1}$ sur $\bar{\Omega} \cap\left\{c_{1}<\rho_{1} \leq 0\right\} \cap\left\{c_{2}<\rho_{2} \leq 0\right\}, Z_{t}^{r}$ et $Z^{r}$ sont continues sur $\bar{\Omega} \backslash D$ puisqu'il est inclus dans l'ensemble précédent.

\section{Lemme 4.15.}

$$
\int_{\Omega \backslash D}\left|\partial \rho_{1} \wedge \partial \rho_{2}(\zeta)\right|^{2} \sigma(\zeta) d \lambda(\zeta) \lesssim B_{1}^{\prime}(\Theta)+B_{2}^{\prime}(\Theta)
$$

Preuve: L'intégrale ne porte que sur les $\zeta$ de $\Omega \backslash D$ tels que $\partial \rho_{1} \wedge$ $\partial \rho_{2}(\zeta) \neq 0$. En complétant $\left(\partial \rho_{1}, \partial \rho_{2}\right)$ dans l'ensemble des $(1,0)$-formes, nous avons $\left(\partial \rho_{1}, \partial \rho_{2}, \gamma_{3}, \ldots, \gamma_{n}\right)$ une base de $(1,0)$-formes sur $\mathbb{C}^{n}$ et $\left(e_{1}(\zeta), \ldots, e_{n}(\zeta)\right)$ une base duale. De plus $\left|\partial \rho_{1}\right|$ et $\left|\partial \rho_{2}\right|$ sont minorables par des constantes absolues sur $V_{1}^{\delta} \cap V_{2}^{\delta}$, ainsi en tant que mesure

$$
\begin{gathered}
\left|\partial \rho_{1} \wedge \partial \rho_{2}(\zeta)\right|^{2} \sum_{k=2}^{n} \Theta\left(e_{k}(\zeta), e_{k}(\zeta)\right) \beta^{n} \lesssim i \partial \rho_{1} \wedge \bar{\partial} \rho_{1} \wedge \Theta \wedge \beta^{n-2}(\zeta), \\
\left|\partial \rho_{1} \wedge \partial \rho_{2}(\zeta)\right|^{2} \sum_{\substack{k=1 \\
k \neq 2}}^{n} \Theta\left(e_{k}(\zeta), e_{k}(\zeta)\right) \beta^{n} \lesssim i \partial \rho_{2} \wedge \bar{\partial} \rho_{2} \wedge \Theta \wedge \beta^{n-2}(\zeta),
\end{gathered}
$$

La trace d'une matrice étant le même quelque soit la base dans laquelle on l'exprime, le Lemme est établi.

Preuve de la Proposition 4.13: Nous allons faire systématiquement le changement de variable $\xi=F_{t}^{r}(\zeta)$, ce qui nous permettra avec les Lemmes précédents de gagner $\mathrm{du}-\rho(\zeta)$ dans chaque terme. 
Grâce au Lemme 4.14, les inégalités (a) et (b) s'obtiennent comme celles de la Proposition 3.3 où le changement de variables $\xi=t \zeta$ est remplacé par $\xi=F_{t}^{r}(\zeta)$ et l'intégration selon la variable $r$ ne joue aucun rôle.

Inégalité (c).

$$
\begin{aligned}
\int_{\Omega}-\frac{1}{\rho_{1}+\rho_{2}} & (\zeta)\left|\partial \rho_{1} \wedge \partial \rho_{2}(\zeta)\right|\left|\bar{\partial} \rho_{k} \wedge\left(w_{1}\right)_{0,1}(\zeta)\right| d \lambda(\zeta) \\
\leq & \int_{\tilde{D}} \int_{0}^{1} \int_{t_{0}}^{1}-\frac{1}{\rho_{1}+\rho_{2}}\left|\left(F_{t}^{r}\right)^{*}\left(\partial \rho_{1} \wedge \partial \rho_{2}\right)\right| \\
& \times\left|\left(F_{t}^{r}\right)^{*}\left(\bar{\partial} \rho_{k} \wedge\left(i_{Z_{t}^{r}} \Theta^{1}\right)_{0,1}\right)\right|(\zeta) d t d r d \lambda(\zeta) \\
& +\int_{\tilde{D}} \int_{0}^{1} \int_{t_{0}}^{1}-\frac{1}{\rho_{1}+\rho_{2}}\left|\left(F_{t}^{r}\right)^{*}\left(i_{Z_{t}^{r}} \Theta^{1}\right)_{0,1}\right| \\
\times\left(\left|\partial \rho_{1} \wedge \partial \rho_{2}\right| \mid\right. & \left.\bar{\partial} \rho_{k}|-|\left(F_{t}^{r}\right)^{*}\left(\partial \rho_{1} \wedge \partial \rho_{2}\right)||\left(F_{t}^{r}\right)^{*}\left(\bar{\partial} \rho_{k}\right) \mid\right)(\zeta) d t d r d \lambda(\zeta) .
\end{aligned}
$$

Nous commençons par majorer le deuxième terme. $\rho_{k}(k=1,2)$ étant une fonction $\mathcal{C}^{3}$ sur $\bar{\Omega}$,

$\forall \zeta \in \tilde{D}, \forall t \in\left[t_{0}, 1\right], \forall r \in[0,1],\left|\bar{\partial} \rho_{k}(\zeta)-\left(F_{t}^{r}\right)^{*}\left(\bar{\partial} \rho_{k}\right)(\zeta)\right| \lesssim\left|\zeta-F_{t}^{r}(\zeta)\right|$

Or $\zeta=F_{1}^{r}(\zeta)$ et la famille de difféomorphismes $\left(F_{t}^{r}\right)$ dépend de façon $\mathcal{C}^{1}$ de $t$ sur $\left[t_{0}, 1\right]$. Ainsi pour tout $t$ de $\left[t_{0}, 1\right]$, tout $r$ de $[0,1]$, tout $\zeta$ de $\tilde{D}$

$$
\left|\zeta-F_{t}^{r}(\zeta)\right| \lesssim 1-t
$$

Nous en déduisons, après le changement de variable $\xi=F_{t}^{r}(\zeta)$, que le deuxième terme est majoré, à une constante absolue près, par

$$
\int_{\Omega \backslash D} \sum_{i, j=1,2}\left|\Theta_{i j}^{1}(\xi)\right| d \lambda(\xi)\left(\int_{0}^{1} \int_{t_{r}(\xi)}^{1}-(1-t) \frac{1}{\rho_{1}+\rho_{2}}\left(\left(F_{t}^{r}\right)^{-1}(\xi)\right) d t d r\right) .
$$

A priori nous ne pouvons plus majorer la somme ci-dessus par la trace du courant puisque $\Theta^{1}$ n'est pas positif sur $D^{\prime} \backslash D$. Mais

$$
\Theta^{1}=\Theta-i d w_{2}=(1-\psi) \Theta-i d \psi \wedge \tilde{w}
$$

$\psi$ étant à valeurs dans $[0,1],(1-\psi) \Theta$ est encore un courant positif majoré par $\Theta$ et avec l'inégalité (4.17) et le Lemme 4.12, nous obtenons que le deuxième terme est majoré, à une constante absolue près, par $B_{0}(\Theta)$. 
Après changement de variable $\xi=F_{t}^{r}(\zeta)$, le premier terme est majoré, à une constante absolue près, par

$$
\begin{aligned}
\int_{\Omega \backslash D} \int_{0}^{1}\left|\partial \rho_{1} \wedge \partial \rho_{2}(\xi)\right| & \left|\bar{\partial} \rho_{k} \wedge\left(i_{Z^{r}} \Theta^{1}\right)_{0,1}(\xi)\right| \\
& \times\left(\int_{t_{r}(\xi)}^{1}-\frac{1}{\rho_{1}+\rho_{2}}\left(\left(F_{t}^{r}\right)^{-1}(\xi)\right) d t\right) d r d \lambda(\xi) .
\end{aligned}
$$

Avec le Lemme 4.11, ceci est majoré à une constante absolue près par

$$
\int_{\Omega \backslash D}\left|\partial \rho_{1} \wedge \partial \rho_{2}(\xi)\right|\left(\int_{0}^{1}\left|\bar{\partial} \rho_{k} \wedge\left(i_{Z^{r}} \Theta^{1}\right)_{0,1}(\xi)\right| d r\right) d \lambda(\xi) .
$$

En appliquant l'inégalité de Cauchy-Schwarz à $(1-\psi) \Theta$, nous avons

$$
\begin{aligned}
\left|\partial \rho_{1} \wedge \partial \rho_{2}(\xi)\right|\left|\bar{\partial} \rho_{k} \wedge\left(i_{Z^{r}}(1-\psi) \Theta\right)_{0,1}(\xi)\right| \\
\quad \leq\left|\partial \rho_{k} \wedge \partial \rho_{2}(\xi)\right|^{2}\left|Z^{r}(\xi)\right|^{2} \sigma(\xi)+\left|\partial \rho_{k} \wedge \bar{\partial} \rho_{k} \wedge \Theta \wedge \beta^{n-2}(\xi)\right| .
\end{aligned}
$$

Avec (4.27), (4.17) et les Lemmes 4.14 et 4.15, l'inégalité (c) est établie. L'inégalité (e) se traite de la même façon.

Inégalité $(d) .-{\overline{\partial\left(w_{1}\right)_{0,1}}}+\partial\left(w_{1}\right)_{0,1}=d w_{1}=-i(1-\psi) \Theta-d \psi \wedge \tilde{w}$. Puis avec (4.17) et l'inégalité de Cauchy-Schwarz appliquée à $\Theta$, nous obtenons l'inégalité (d).

Inégalité (f). $\rho_{1}$ et $\rho_{2}$ étant $\mathcal{C}^{3}$ avec (4.26) nous avons encore

$$
\begin{gathered}
\int_{\Omega} \frac{\left(-\rho_{1}\right)^{1 / 2}\left(-\rho_{2}\right)^{1 / 2}}{\left(-\rho_{1}-\rho_{2}\right)^{5 / 2}}(\zeta)\left|\partial \rho_{1} \wedge \partial \rho_{2}(\zeta)\right|\left|\bar{\partial} \rho_{1} \wedge \bar{\partial} \rho_{2} \wedge\left(w_{1}\right)_{0,1}(\zeta)\right| d \lambda(\zeta) \\
\leq \int_{\tilde{D}} \int_{0}^{1} \int_{t_{0}}^{1} \frac{\left(-\rho_{1}\right)^{1 / 2}\left(-\rho_{2}\right)^{1 / 2}}{\left(-\rho_{1}-\rho_{2}\right)^{5 / 2}}(\zeta)\left|\left(F_{t}^{r}\right)^{*}\left(\partial \rho_{1} \wedge \partial \rho_{2}\right)\right| \\
\times\left|\left(F_{t}^{r}\right)^{*}\left(\bar{\partial} \rho_{1} \wedge \bar{\partial} \rho_{2} \wedge\left(i_{Z_{t}^{r}} \Theta^{1}\right)_{0,1}\right)\right|(\zeta) d t d r d \lambda(\zeta) \\
\quad+\int_{\tilde{D}} \int_{0}^{1} \int_{t_{0}}^{1} \frac{\left(-\rho_{1}\right)^{1 / 2}\left(-\rho_{2}\right)^{1 / 2}}{\left(-\rho_{1}-\rho_{2}\right)^{5 / 2}}(\zeta)(1-t) \\
\quad \times \sum_{k=1,2}\left|\left(F_{t}^{r}\right)^{*}\left(\bar{\partial} \rho_{k} \wedge\left(i_{Z_{t}^{r}} \Theta^{1}\right)_{0,1}\right)\right| d t d r d \lambda(\zeta) \\
+\int_{\tilde{D}} \int_{0}^{1} \int_{t_{0}}^{1} \frac{\left(-\rho_{1}\right)^{1 / 2}\left(-\rho_{2}\right)^{1 / 2}}{\left(-\rho_{1}-\rho_{2}\right)^{5 / 2}}(\zeta)(1-t)^{2}\left|\left(F_{t}^{r}\right)^{*}\left(i_{Z_{t}^{r}} \Theta^{1}\right)_{0,1}\right| d t d r d \lambda(\zeta) .
\end{gathered}
$$


Avec (4.27), (4.17) et le Lemme 4.12, le deuxième et le troisième termes sont majorés, à une constante absolue près, par $B_{0}(\Theta)+B_{1}^{\prime}(\Theta)+B_{2}^{\prime}(\Theta)$.

Après changement de variable $\xi=F_{t}^{r}(\zeta)$, le premier terme est majoré, à une constante absolue près, par

$$
\begin{aligned}
\int_{\Omega \backslash D} & \int_{0}^{1}\left|\partial \rho_{1} \wedge \partial \rho_{2}(\xi)\right|\left|\bar{\partial} \rho_{1} \wedge \bar{\partial} \rho_{2} \wedge\left(i_{Z^{r}} \Theta^{1}\right)_{0,1}(\xi)\right| \\
& \times\left(\int_{t_{r}(\xi)}^{1} \frac{\left(-\rho_{1}\right)^{1 / 2}\left(-\rho_{2}\right)^{1 / 2}}{\left(-\rho_{1}-\rho_{2}\right)^{5 / 2}}\left(\left(F_{t}^{r}\right)^{-1}(\xi)\right) d t\right) d r d \lambda(\xi)
\end{aligned}
$$

Comme pour les Lemmes 4.10 et 4.11, nous sommes amenés à distinguer trois cas.

- $\xi \in(\Omega \backslash D) \cap\left\{-\rho_{1} \leq \frac{c_{1}^{\prime}}{c_{2}}\left(-\rho_{2}\right)\right\}$.

Avec le Lemme 4.10 et le Corollaire 4.9, l'intégrale (4.28) restreinte à l'ensemble ci-dessus est majorée, à une constante absolue près, par

$$
\begin{aligned}
& \int\left\{-\rho_{1} \leq \frac{c_{1}^{\prime}}{c_{2}}\left(-\rho_{2}\right)\right\} \frac{\left(-\rho_{1}\right)^{3 / 2}\left(-\rho_{2}\right)^{3 / 2}}{\left(-\rho_{1}-\rho_{2}\right)^{7 / 2}}(\xi)\left|\partial \rho_{1} \wedge \partial \rho_{2}(\xi)\right| \\
& \times\left(\int_{0}^{1}\left|\bar{\partial} \rho_{1} \wedge \bar{\partial} \rho_{2} \wedge\left(i_{Z^{r}} \Theta^{1}\right)_{0,1}(\xi)\right| d r\right) d \lambda(\xi)
\end{aligned}
$$

En appliquant l'inégalité de Cauchy-Schwarz à $(1-\psi) \Theta$

$$
\begin{aligned}
\frac{\left(-\rho_{1}\right)^{3 / 2}\left(-\rho_{2}\right)^{3 / 2}}{\left(-\rho_{1}-\rho_{2}\right)^{7 / 2}}(\xi)\left|\partial \rho_{1} \wedge \partial \rho_{2}(\xi)\right|\left|\bar{\partial} \rho_{1} \wedge \bar{\partial} \rho_{2} \wedge\left(i_{Z^{r}}(1-\psi) \Theta^{1}\right)_{0,1}(\xi)\right| \\
\quad \lesssim\left|\partial \rho_{1} \wedge \partial \rho_{2}(\xi)\right|^{2}\left|Z^{r}(\xi)\right|^{2} \sigma(\xi) \\
\quad+\frac{\left(-\rho_{1}\right)^{3}\left(-\rho_{2}\right)^{3}}{\left(-\rho_{1}-\rho_{2}\right)^{7}}(\xi)\left|\partial \rho_{1} \wedge \bar{\partial} \rho_{1} \wedge \partial \rho_{2} \wedge \bar{\partial} \rho_{2} \wedge \Theta \wedge \beta^{n-3}(\xi)\right|
\end{aligned}
$$

L'intégrale (4.29) est majorée à une constante absolue près par $B_{0}(\Theta)+$ $B_{12}(\Theta)$.

$$
\text { - } \xi \in(\Omega \backslash D) \cap\left\{-\rho_{2} \leq \frac{c_{2}^{\prime}}{c_{1}}\left(-\rho_{1}\right)\right\} \text {. }
$$

Analogue au cas précédent en échangeant les rôles de $\left(-\rho_{1}\right)$ et $\left(-\rho_{2}\right)$.

$$
\text { - } \xi \in(\Omega \backslash D) \cap\left\{\frac{c_{2}^{\prime}}{c_{1}}\left(-\rho_{1}\right) \leq-\rho_{2} \leq \frac{c_{2}}{c_{1}^{\prime}}\left(-\rho_{1}\right)\right\}
$$

Les majorations sont les mêmes.

Les trois cas envisagés ci-dessus, nous permettent d'établir l'inégalité (f) et la preuve de la Proposition 4.13 est terminée. 
4.4 Condition suffisante d'appartenance à $N_{2}(\Omega)$.

Théorème 4.16. Si le $(1,1)$-courant positif et fermé $\Theta$ vérifie :

$$
\begin{aligned}
& B_{0}(\Theta)=\int_{\Omega}-\rho \Theta \wedge \beta^{n-1}<+\infty \\
& B_{k}^{\prime}(\Theta)=\int_{\Omega} i \partial \rho_{k} \wedge \bar{\partial} \rho_{k} \wedge \Theta \wedge \beta^{n-2}<+\infty \text { pour } k=1,2,
\end{aligned}
$$

et pour $n \geq 3$

$B_{12}^{\prime}(\Theta)=\int_{\Omega}-\frac{1}{\rho}\left(\frac{\rho}{\rho_{1}}\right)^{3}\left(\frac{\rho}{\rho_{2}}\right)^{3} i \partial \rho_{1} \wedge \bar{\partial} \rho_{1} \wedge i \partial \rho_{2} \wedge \bar{\partial} \rho_{2} \wedge \Theta \wedge \beta^{n-3}<+\infty$,

alors il existe une fonction plurisousharmonique $W$ dans la classe de Nevanlinna $N_{2}(\Omega)$, solution dans $\Omega$ de l'équation $i \partial \bar{\partial} W=\Theta$.

Preuve: Lorsque $\Theta$ est un courant à coefficients dans $\mathcal{C}^{2}(\bar{\Omega})$, avec le Théorème 4.3 et les Propositions 4.4, 4.13 nous pouvons définir, selon les égalités (4.14) et (4.20), $w=w_{1}+w_{2}$ solution de $i d w=\Theta$ et tel que la solution de $\bar{\partial} U=w_{0,1}$ donnée par la Proposition-Définition 4.1 satisfasse

$$
\int_{S_{12}}|U| i \bar{\partial} \rho_{1} \wedge i \bar{\partial} \rho_{2} \wedge \beta^{n-2} \lesssim B_{0}(\Theta)+B_{1}^{\prime}(\Theta)+B_{2}^{\prime}(\Theta)\left(+B_{12}(\Theta)\right)
$$

Nous mettons $B_{12}(\Theta)$ entre parenthèses car il n'apparait que pour $n \geq 3$.

En outre, comme dans le paragraphe III, nous avons

$$
\int_{S_{k}}|U| d \sigma_{k} \lesssim B_{0}(\Theta)+B_{1}^{\prime}(\Theta)+B_{2}^{\prime}(\Theta) \text { pour } k=1,2 .
$$

Montrons que $\forall \varepsilon>0, \int_{\partial \Omega_{\varepsilon}} W^{+} i \bar{\partial} \rho \wedge(i \partial \bar{\partial} \rho)^{n-1} \lesssim \int_{S_{12}}|W| i \bar{\partial} \rho_{1} \wedge i \bar{\partial} \rho_{2} \wedge$ $\beta^{n-2}+\sum_{k=1,2} \int_{S_{k}}|W| d \sigma_{k}$. Pour cela nous suivons les mêmes étapes que la preuve de la Proposition 3.4.

$1^{e}$ étape. Posons pour $\eta>0, D_{\eta}=\left\{z \in \mathbb{C}^{n} \mid \rho_{1}(z)+\rho_{2}(z)<-\eta\right\}$,

$$
\begin{aligned}
\forall \varepsilon>0 \int_{\partial \Omega_{\varepsilon}} W^{+} i \bar{\partial} \rho & \wedge(i \partial \bar{\partial} \rho)^{n-1} \\
& \leq \lim _{\eta \downarrow 0} \int_{\Omega \cap D_{\eta}}\left(W^{+} i \partial \bar{\partial} \rho-\rho i \partial \bar{\partial} W^{+}\right) \wedge(i \partial \bar{\partial} \rho)^{n-1},
\end{aligned}
$$


$2^{e}$ étape.

$$
\begin{aligned}
\lim _{\eta \downarrow 0} \int_{\Omega \cap D_{\eta}}\left(W^{+}\right. & \left.i \partial \bar{\partial} \rho-\rho i \partial \bar{\partial} W^{+}\right) \wedge(i \partial \bar{\partial} \rho)^{n-1} \\
& \lesssim \sum_{j=1,2} \int_{S_{j}} W^{+} d \sigma_{j}+\int_{S_{12}} W^{+} i \bar{\partial} \rho_{1} \wedge i \bar{\partial} \rho_{2} \wedge \beta^{n-2}
\end{aligned}
$$

Après avoir régularisé $W^{+}$et fait un passage à la limite, nous obtenons

$$
\begin{aligned}
\lim _{\eta \downarrow 0} \int_{\Omega \cap D_{\eta}}\left(W^{+} i \partial \bar{\partial} \rho-\rho i \partial \bar{\partial} W^{+}\right) \wedge(i \partial \bar{\partial} \rho)^{n-1} & \\
& =\lim _{\eta \downarrow 0} \int_{\partial\left(\Omega \cap D_{\eta}\right)} W^{+} i \bar{\partial} \rho \wedge(i \partial \bar{\partial} \rho)^{n-1}
\end{aligned}
$$

L'intégrale sur $\partial \Omega \cap D_{\eta}$ est majorée par la somme des intégrales de $W^{+}$ sur les faces $S_{1}$ et $S_{2}$. Par contre puisque $\left|i \bar{\partial} \rho \wedge(i \partial \bar{\partial} \rho)^{n-1}\right|$ n'est pas borné sur $\Omega$ nous devons considérer l'intégrale sur $\partial D_{\eta}$.

$$
\begin{aligned}
\partial D_{\eta} & =\left\{z \mid\left(\rho_{1}+\rho_{2}\right)(z)=-\eta\right\} \\
i \bar{\partial} \rho \wedge(i \partial \bar{\partial} \rho)^{n-1} & =w^{n-1} \wedge i \bar{\partial} \rho+(n-1) w^{n-2} \wedge w_{1,2}
\end{aligned}
$$

avec

$$
\begin{aligned}
w & =\left(\frac{\rho}{\rho_{1}}\right)^{2} i \partial \bar{\partial} \rho_{1}+\left(\frac{\rho}{\rho_{2}}\right)^{2} i \partial \bar{\partial} \rho_{2} \\
w_{1,2} & =-\frac{2}{\rho_{1}+\rho_{2}}\left(\frac{\rho}{\rho_{1}}\right)\left(\frac{\rho}{\rho_{2}}\right)\left(\frac{\rho}{\rho_{2}} d \rho_{2}-\frac{\rho}{\rho_{1}} d \rho_{1}\right) \wedge i \bar{\partial} \rho_{1} \wedge i \bar{\partial} \rho_{2} .
\end{aligned}
$$

$w$ et $i \bar{\partial} \rho$ sont à coefficients bornés sur $\Omega$, d'où

$$
\lim _{\eta \downarrow 0} \int_{\left(\partial D_{\eta}\right) \cap \Omega} W^{+} w^{n-1} \wedge i \bar{\partial} \rho=0 .
$$

$\operatorname{Sur}\left\{\rho_{1}+\rho_{2}=-\eta\right\}, w_{1,2} \equiv 2 \frac{\rho_{1} \rho_{2}}{\eta^{3}} d \rho_{1} \wedge i \bar{\partial} \rho_{1} \wedge i \bar{\partial} \rho_{2}$ et $\frac{2}{\eta^{3}} \int_{-\eta}^{0}-\rho_{1}(\eta+$ $\left.\rho_{1}\right) d \rho_{1}=\frac{1}{3}$. Après avoir pris $\rho_{1}, \rho_{2}$ et $\xi \in S_{12}$ comme coordonnées locales et utilisé la continuité de $W^{+}$, nous obtenons

$$
\lim _{\eta \downarrow 0} \int_{\left(\partial D_{\eta}\right) \cap \Omega} W^{+} w^{n-2} \wedge w_{1,2} \lesssim \int_{S_{12}} W^{+} i \bar{\partial} \rho_{1} \wedge i \bar{\partial} \rho_{2} \wedge \beta^{n-2}
$$

Avec les relations (4.32) à (4.34) la $2^{e}$ étape est établie ainsi que la Proposition dans le cas $\mathcal{C}^{2}$. Nous terminons en régularisant comme dans le paragraphe 3.2 . 


\section{Bibliographie}

[And] M. Andersson, Solution formulas for the $\partial \bar{\partial}$-equation and weighted Nevanlinna classes in the polydisc, Bull. Sc. Math., $2^{e}$ série 109 (1985), 135-154.

[B.A] B. Berndtsson And M. Andersson, Henkin-Ramirez formulas with weight factors, Ann. Inst. Fourier 32 (1982), 91-110.

[Ber] B. Berndtsson, A formula for interpolation and division in $\mathbb{C}^{n}$, Math. Ann. 263 (1983), 399-418.

[C.N.S] D-C. Chang, A. Nagel And E. Stein, Estimates for the $\bar{\partial}$-Neumann problem in pseudoconvex domains of finite type in $\mathbb{C}^{2}$, Acta Math. 169 (1992), 153-228.

[Cha] P. Charpentier, Caractérisation des zéros des fonctions de certaines classes de type Nevanlinna dans le bidisque, Ann. Inst. Fourier 34 (1984), 57-98.

[C.D] P. Charpentier and Y. Dupain, Une estimation des coefficients tangents d'un courant positif fermé dans un domaine de $\mathbb{C}^{3}$, Publicacions Matemàtiques 36 (1992), 319-349.

[Dem 1] J. P. Demailly, Mesures de Monge-Ampère et caractérisation géométrique des variétés algébriques affines, Mémoire Soc. Math. France 19 (1985).

[Dem 2] J. P. Demailly, Mesures de Monge-Ampère et mesures pluriharmoniques, Math. Z. 194 (1987), 519-564.

[For] J. E. FornaEss, Embedding strictly pseudoconvex domains in convex domains, Amer. J. of Math. 98 (1976), 529-569.

[Hen] G. M. Henkin, Solutions with estimates of the H. Levy and Poincaré-Lelong equations. Construction of functions of the Nevanlinna class with prescribed zeros in strictly pseudoconvex domains., Dokl. Akad. Nauk. SSSR 224 (1975), 3-13.

[Men] C. Menini, "Classes de Nevanlinna et estimations pour la résolution de l'équation de Cauchy-Riemann sur une intersection d'ouverts strictement pseudoconvexes," Thèse $n^{\circ} 1712$, Toulouse III, 1994.

[R.S] R. M. Range And Y. T. SiU, Uniform Estimates for the $\bar{\partial}$-Equation on Domains with Piecewise Smooth Strictly Pseudoconvex Boundaries, Math. Ann. 206 (1973), 325-354.

[Sko] H. SkodA, Valeurs au bord pour les solutions de l'opérateur d" et caractérisation des zéros des fonctions de la classe de Nevanlinna, Bull. Soc. Math. France (1976), 225-299. 
[Var] N. VAropoulos, BMO functions and the $\bar{\partial}$-equation, Pacific J. Math. 71 (1977), 221-273.

\author{
Université de Bordeaux I \\ UFR de Mathematiques \\ Laboratoire de Mathematiques Pures \\ 351 cours de la Liberation \\ 33405 Talence Cedex \\ FRANCE
}

Rebut el 3 d'Octubre de 1994 